\title{
Isolated and Dynamical Horizons and Their Applications
}

\author{
Abhay Ashtekar \\ Institute for Gravitational Physics and Geometry \\ Pennsylvania State University \\ University Park, PA 16801, U.S.A. \\ and \\ Kavli Institute of Theoretical Physics \\ University of California \\ Santa Barbara, CA 93106-4030, U.S.A. \\ and \\ Max-Planck-Institut für Gravitationsphysik, Albert-Einstein-Institut \\ Am Mühlenberg 1, 14476 Golm, Germany \\ and \\ Erwin-Schrödinger-Institut \\ Boltzmanngasse 9, 1090 Vienna, Austria \\ email: ashtekar@gravity.psu.edu \\ http://cgpg.gravity.psu.edu/people/Ashtekar/ \\ Badri Krishnan \\ Max-Planck-Institut für Gravitationsphysik, Albert-Einstein-Institut \\ Am Mühlenberg 1, 14476 Golm, Germany \\ and \\ Erwin-Schrödinger-Institut \\ Boltzmanngasse 9, 1090 Vienna, Austria \\ email: badri.krishnan@aei.mpg.de \\ Accepted on 23 November 2004 \\ Published on 15 December 2004 \\ http://www.livingreviews.org/lrr-2004-10 \\ Living Reviews in Relativity \\ Published by the Max Planck Institute for Gravitational Physics \\ Albert Einstein Institute, Germany
}

\begin{abstract}
Over the past three decades, black holes have played an important role in quantum gravity, mathematical physics, numerical relativity and gravitational wave phenomenology. However, conceptual settings and mathematical models used to discuss them have varied considerably from one area to another. Over the last five years a new, quasi-local framework was introduced to analyze diverse facets of black holes in a unified manner. In this framework, evolving black holes are modelled by dynamical horizons and black holes in equilibrium by isolated horizons. We review basic properties of these horizons and summarize applications to mathematical
\end{abstract}

(C) Max Planck Society and the authors.

Further information on copyright is given at http://relativity.livingreviews.org/Info/Copyright/

For permission to reproduce the article please contact livrev@aei.mpg.de. 
physics, numerical relativity, and quantum gravity. This paradigm has led to significant generalizations of several results in black hole physics. Specifically, it has introduced a more physical setting for black hole thermodynamics and for black hole entropy calculations in quantum gravity, suggested a phenomenological model for hairy black holes, provided novel techniques to extract physics from numerical simulations, and led to new laws governing the dynamics of black holes in exact general relativity.

Living Reviews in Relativity

http: //www . livingreviews . org/lrr-2004-10 


\section{Article Amendments}

On author request a Living Reviews article can be amended to include errata and small additions to ensure that the most accurate and up-to-date information possible is provided. If an article has been amended, a summary of the amendments will be listed on this page. For detailed documentation of amendments, please refer always to the article's online version at http://www. livingreviews.org/lrr-2004-10.

\section{How to cite this article}

Owing to the fact that a Living Reviews article can evolve over time, we recommend to cite the article as follows:

Abhay Ashtekar and Badri Krishnan,

"Isolated and Dynamical Horizons and Their Applications",

Living Rev. Relativity, 7, (2004), 10. [Online Article]: cited [<date $>$ ],

http://www.livingreviews.org//rr-2004-10

The date given as < date $>$ then uniquely identifies the version of the article you are referring to. 


\section{Contents}

1 Introduction $\quad 7$

2 Basic Notions $\quad 14$

2.1 Isolated horizons . . . . . . . . . . . . . . . . . . . . . . . 14

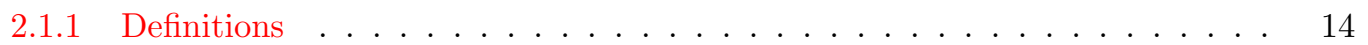

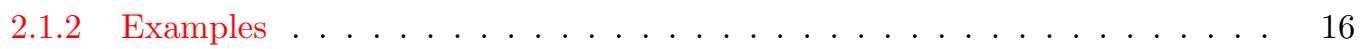

2.1 .3 Geometrical properties . . . . . . . . . . . . . . . . . . . 17

2.2 Dynamical horizons . . . . . . . . . . . . . . . . . . . . . . . . . . . . . . . . . . . . . . . . . . .

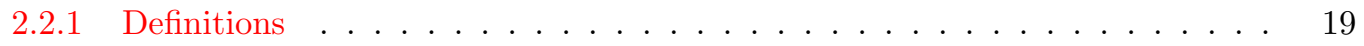

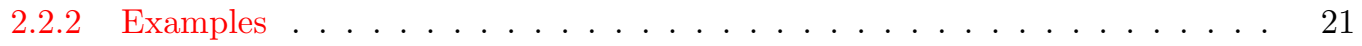

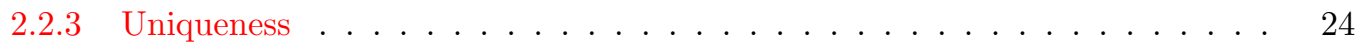

3 Area Increase Law 26

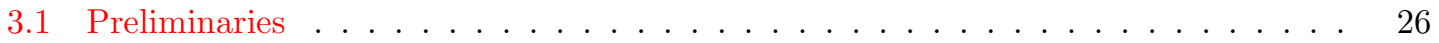

3.2 Area increase law . . . . . . . . . . . . . . . . . . . . . . 27

3.3 Energy flux due to gravitational waves . . . . . . . . . . . . . . . . . . . 29

4 Black Hole Mechanics $\quad 32$

4.1 Mechanics of weakly isolated horizons . . . . . . . . . . . . . . . . . . . 32

4.1 The zeroth law . . . . . . . . . . . . . . . . . . . 32

4.1.2 Phase space, symplectic structure, and angular momentum . . . . . . . 33

4.1 .3 Energy, mass, and the first law . . . . . . . . . . . . . . . . . 35

4.2 Mechanics of dynamical horizons . . . . . . . . . . . . . . . . . . . . . . . . . . . . . . . . . . . . . . . .

4.2.1 Angular momentum balance. . . . . . . . . . . . . . . . . . . . . . . . . . . . . . . . . . 38

4.2 .2 Integral form of the first law . . . . . . . . . . . . . . . . . . . . . . . . . . . . . . .

4.2 .3 Horizon mass . . . . . . . . . . . . . . . . . . . . . . . . . . . . . . . . . . . . . .

4.3 Passage of dynamical horizons to equilibrium . . . . . . . . . . . . . . . . . 42

5 Applications in Numerical Relativity $\quad 43$

5.1 Numerical computation of black hole mass and angular momentum . . . . . . . . . 43

5.2 Initial data . . . . . . . . . . . . . . . . . . . . . . . 46

5.2 .1 Boundary conditions at the inner boundary . . . . . . . . . . . . . 47

5.2 .2 Binding energy . . . . . . . . . . . . . . . . . . . . . . . . . . . . . . . . . . . . 49

5.3 Black hole multipole moments . . . . . . . . . . . . . . . . . . . . . . . 51

5.4 Waveform extraction . . . . . . . . . . . . . . . . . . 52

6 Applications in Mathematical Physics $\quad 55$

6.1 Beyond Einstein-Maxwell theory . . . . . . . . . . . . . . . . . . . . . 55

6.1 .1 Dilatonic couplings . . . . . . . . . . . . . . . . . . 55

6.1 .2 Yang-Mills fields . . . . . . . . . . . . . . . . . . 57

6.2 Structure of colored, static black holes . . . . . . . . . . . . . . . . . 59

6.2 .1 Horizon mass . . . . . . . . . . . . . . . . . . . 59

6.2.2 Phenomenological model of colored black holes . . . . . . . . . . . . . 60

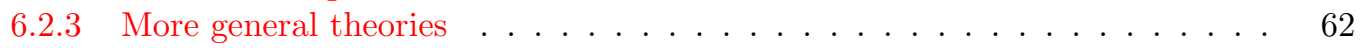

7 Applications in Quantum Gravity $\quad 64$

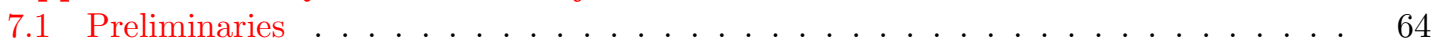

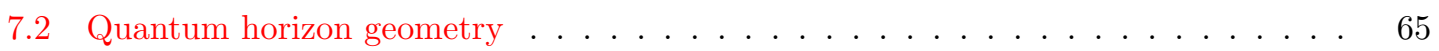

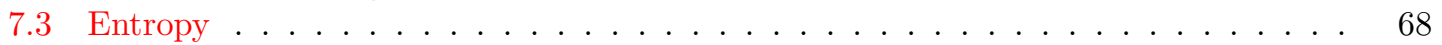


9 Acknowledgements 



\section{Introduction}

Research inspired by black holes has dominated several areas of gravitational physics since the early seventies. The mathematical theory turned out to be extraordinarily rich and full of surprises. Laws of black hole mechanics brought out deep, unsuspected connections between classical general relativity, quantum physics, and statistical mechanics [35, 44, 45, 46]. In particular, they provided a concrete challenge to quantum gravity which became a driving force for progress in that area. On the classical front, black hole uniqueness theorems $[69,119]$ took the community by surprise. The subsequent analysis of the detailed properties of Kerr-Newman solutions [65] and perturbations thereof [66] constituted a large fraction of research in mathematical general relativity in the seventies and eighties. Just as the community had come to terms with the uniqueness results, it was surprised yet again by the discovery of hairy black holes [38, 49]. Research in this area continues to be an active branch of mathematical physics [181]. The situation has been similar in numerical relativity. Since its inception, much of the research in this area has been driven by problems related to black holes, particularly their formation through gravitational collapse [153], the associated critical phenomenon [67, 105], and the dynamics leading to their coalescence (see, e.g., $[1,126,160,58,2,138])$. Finally, black holes now play a major role in relativistic astrophysics, providing mechanisms to fuel the most powerful engines in the cosmos. They are also among the most promising sources of gravitational waves, leading to new avenues to confront theory with experiments [78].

Thus there has been truly remarkable progress on many different fronts. Yet, as the subject matured, it became apparent that the basic theoretical framework has certain undesirable features from both conceptual and practical viewpoints. Nagging questions have persisted, suggesting the need of a new paradigm.

\section{Dynamical situations}

For fully dynamical black holes, apart from the 'topological censorship' results which restrict the horizon topology [110, 90], there has essentially been only one major result in exact general relativity. This is the celebrated area theorem proved by Hawking in the early seventies [111, 113]: If matter satisfies the null energy condition, the area of the black hole event horizon can never decrease. This theorem has been extremely influential because of its similarity with the second law of thermodynamics. However, it is a qualitative result; it does not provide an explicit formula for the amount by which the area increases in physical processes. Now, for a black hole of mass $M$, angular momentum $J$, area $a$, surface gravity $\kappa$, and angular velocity $\Omega$, the first law of black hole mechanics,

$$
\delta M=\frac{\kappa}{8 \pi G} \delta a+\Omega \delta J,
$$

does relate the change in the horizon area to that in the energy and angular momentum, as the black hole makes a transition from one equilibrium state to a nearby one [35, 182]. This suggests that there may well be a fully dynamical version of Equation (1) which relates the change in the black hole area to the energy and angular momentum it absorbs in fully dynamical processes in which the black hole makes a transition from a given state to one which is far removed. Indeed, without such a formula, one would have no quantitative control on how black holes grow in exact general relativity. Note however that the event horizons can form and grow even in a flat region of space-time (see Figure 4 and Section 2.2.2 for illustrations). During this phase, the area grows in spite of the fact that there is no flux of energy or angular momentum across the event horizon. Hence, in the standard framework where the surface of the black hole is represented by an event horizon, it is impossible to obtain the desired formula. Is there then a more appropriate notion that can replace event horizons? 


\section{Equilibrium situations}

The zeroth and first laws of black hole mechanics refer to equilibrium situations and small departures therefrom. Therefore, in this context, it is natural to focus on isolated black holes. It was customary to represent them by stationary solutions of field equations, i.e, solutions which admit a time-translational Killing vector field everywhere, not just in a small neighborhood of the black hole. While this simple idealization was natural as a starting point, it is overly restrictive. Physically, it should be sufficient to impose boundary conditions at the horizon which ensure only that the black hole itself is isolated. That is, it should suffice to demand only that the intrinsic geometry of the horizon be time independent, whereas the geometry outside may be dynamical and admit gravitational and other radiation. Indeed, we adopt a similar viewpoint in ordinary thermodynamics; while studying systems such as a classical gas in a box, one usually assumes that only the system under consideration is in equilibrium, not the whole world. In realistic situations, one is typically interested in the final stages of collapse where the black hole has formed and 'settled down' or in situations in which an already formed black hole is isolated for the duration of the experiment (see Figure 1). In such cases, there is likely to be gravitational radiation and non-stationary matter far away from the black hole. Thus, from a physical perspective, a framework which demands global stationarity is too restrictive.
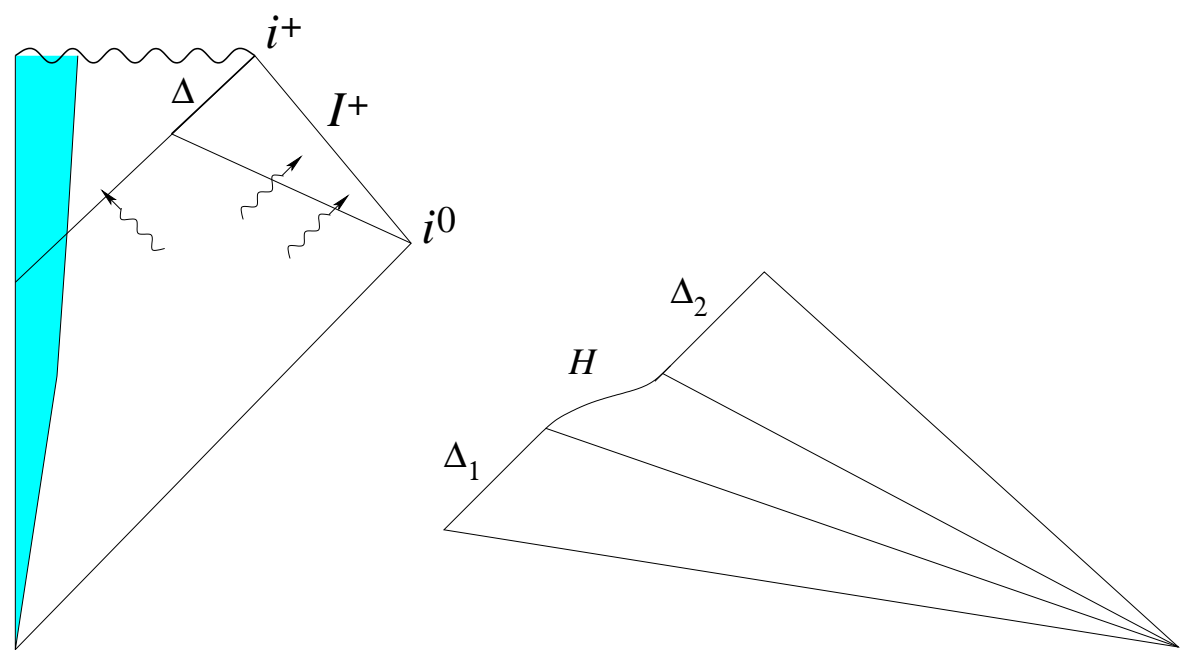

Figure 1: Left panel: A typical gravitational collapse. The portion $\Delta$ of the event horizon at late times is isolated. Physically, one would expect the first law to apply to $\Delta$ even though the entire space-time is not stationary because of the presence of gravitational radiation in the exterior region. Right panel: Space-time diagram of a black hole which is initially in equilibrium, absorbs a finite amount of radiation, and again settles down to equilibrium. Portions $\Delta_{1}$ and $\Delta_{2}$ of the horizon are isolated. One would expect the first law to hold on both portions although the space-time is not stationary.

Even if one were to ignore these conceptual considerations and focus just on results, the framework has certain unsatisfactory features. Consider the central result, the first law of Equation (1). Here, the angular momentum $J$ and the mass $M$ are defined at infinity while the angular velocity $\Omega$ and surface gravity $\kappa$ are defined at the horizon. Because one has to go back and forth between the horizon and infinity, the physical meaning of the first law

Living Reviews in Relativity

http: //www . livingreviews . org/lrr-2004-10 
is not transparent ${ }^{1}$. For instance, there may be matter rings around the black hole which contribute to the angular momentum and mass at infinity. Why is this contribution relevant to the first law of black hole mechanics? Shouldn't only the angular momentum and mass of the black hole feature in the first law? Thus, one is led to ask: Is there a more suitable paradigm which can replace frameworks based on event horizons in stationary space-times?

\section{Entropy calculations}

The first and the second laws suggest that one should assign to a black hole an entropy which is proportional to its area. This poses a concrete challenge to candidate theories of quantum gravity: Account for this entropy from fundamental, statistical mechanical considerations. String theory has had a remarkable success in meeting this challenge in detail for a subclass of extremal, stationary black holes whose charge equals mass (the so-called BPS states) [120]. However, for realistic black holes the charge to mass ratio is less than $10^{-18}$. It has not been possible to extend the detailed calculation to realistic cases where charge is negligible and matter rings may distort the black hole horizon. From a mathematical physics perspective, the entropy calculation should also encompass hairy black holes whose equilibrium states cannot be characterized just by specifying the mass, angular momentum and charges at infinity, as well as non-minimal gravitational couplings, in presence of which the entropy is no longer a function just of the horizon area. One may therefore ask if other avenues are available. A natural strategy is to consider the sector of general relativity containing an isolated black hole and carry out its quantization systematically. A pre-requisite for such a program is the availability of a manageable action principle and/or Hamiltonian framework. Unfortunately, however, if one attempts to construct these within the classical frameworks traditionally used to describe black holes, one runs into two difficulties. First, because the event horizon is such a global notion, no action principle is known for the sector of general relativity containing geometries which admit an event horizon as an internal boundary. Second, if one restricts oneself to globally stationary solutions, the phase space has only a finite number of true degrees of freedom and is thus 'too small' to adequately incorporate all quantum fluctuations. Thus, again, we are led to ask: Is there a more satisfactory framework which can serve as the point of departure for a non-perturbative quantization to address this problem?

\section{Global nature of event horizons}

The future event horizon is defined as the future boundary of the causal past of future null infinity. While this definition neatly encodes the idea that an outside observer can not 'look into' a black hole, it is too global for many applications. First, since it refers to null infinity, it can not be used in spatially compact space-times. Surely, one should be able to analyze black hole dynamics also in these space-times. More importantly, the notion is teleological; it lets us speak of a black hole only after we have constructed the entire space-time. Thus, for example, an event horizon may well be developing in the room you are now sitting in anticipation of a gravitational collapse that may occur in this region of our galaxy a million years from now. When astrophysicists say that they have discovered a black hole in the center of our galaxy, they are referring to something much more concrete and quasi-local than an event horizon. Is there a satisfactory notion that captures what they are referring to?

The teleological nature of event horizons is also an obstruction to extending black hole mechanics in certain physical situations. Consider for example, Figure 2 in which a spherical star of mass $M$ undergoes a gravitational collapse. The singularity is hidden inside the null surface $\Delta_{1}$ at $r=2 M$ which is foliated by a family of marginally trapped surfaces and

\footnotetext{
${ }^{1}$ The situation is even more puzzling in the Einstein-Yang-Mills theory where the right side of Equation (1) acquires an additional term, $V \delta Q$. In treatments based on stationary space-times, not only the Yang-Mills charge $Q$, but also the potential $V$ (the analog of $\Omega$ and $\kappa$, is evaluated at infinity [175].
} 


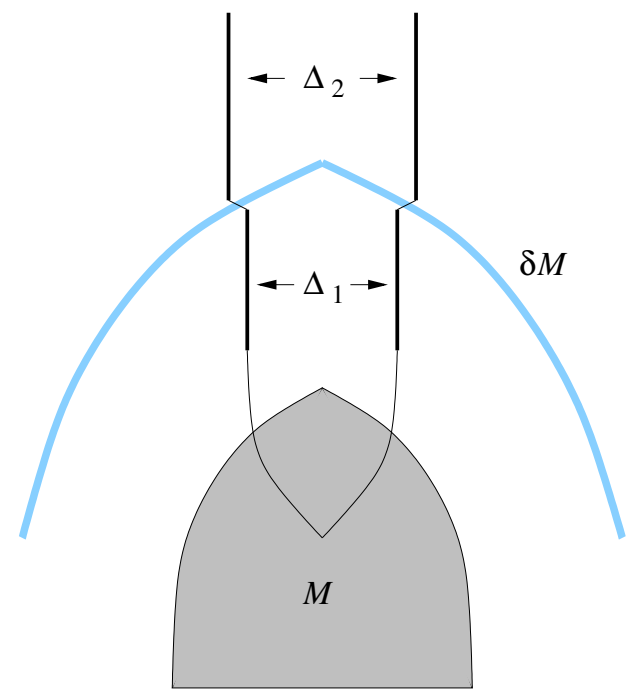

Figure 2: A spherical star of mass $M$ undergoes collapse. Much later, a spherical shell of mass $\delta M$ falls into the resulting black hole. While $\Delta_{1}$ and $\Delta_{2}$ are both isolated horizons, only $\Delta_{2}$ is part of the event horizon.

would be a part of the event horizon if nothing further happens. Suppose instead, after a million years, a thin spherical shell of mass $\delta M$ collapses. Then $\Delta_{1}$ would not be a part of the event horizon which would actually lie slightly outside $\Delta_{1}$ and coincide with the surface $r=2(M+\delta M)$ in the distant future. On physical grounds, it seems unreasonable to exclude $\Delta_{1}$ a priori from thermodynamical considerations. Surely one should be able to establish the standard laws of mechanics not only for the equilibrium portion of the event horizon but also for $\Delta_{1}$.

Next, let us consider numerical simulations of binary black holes. Here the main task is to construct the space-time containing evolving black holes. Thus, one needs to identify initial data containing black holes without the knowledge of the entire space-time and evolve them step by step. The notion of a event horizon is clearly inadequate for this. One uses instead the notion of apparent horizons (see Section 2.2). One may then ask: Can we use apparent horizons instead of event horizons in other contexts as well? Unfortunately, it has not been possible to derive the laws of black hole mechanics using apparent horizons. Furthermore, as discussed in section 2, while apparent horizons are 'local in time' they are still global notions, tied too rigidly to the choice of a space-like 3-surface to be directly useful in all contexts. Is there a truly quasi-local notion which can be useful in all these contexts?

\section{Disparate paradigms}

In different communities within gravitational physics, the intended meaning of the term 'black hole' varies quite considerably. Thus, in a string theory seminar, the term 'fundamental black holes' without further qualification generally refers to the BPS states referred to above $-a$ sub-class of stationary, extremal black holes. In a mathematical physics talk on black holes, the fundamental objects of interest are stationary solutions to, say, the Einstein-Higgs-YangMills equations for which the uniqueness theorem fails. The focus is on the ramifications of 'hair', which are completely ignored in string theory. In a numerical relativity lecture, both these classes of objects are considered to be so exotic that they are excluded from discussion without comment. The focus is primarily on the dynamics of apparent horizons

Living Reviews in Relativity

http: //www . livingreviews . org/lrr-2004-10 
in general relativity. In astrophysically interesting situations, the distortion of black holes by external matter rings, magnetic fields and other black holes is often non-negligible [87, 98, 88]. While these illustrative notions seem so different, clearly there is a common conceptual core. Laws of black hole mechanics and the statistical mechanical derivation of entropy should go through for all black holes in equilibrium. Laws dictating the dynamics of apparent horizons should predict that the final equilibrium states are those represented by the stable stationary solutions of the theory. Is there a paradigm that can serve as an unified framework to establish such results in all these disparate situations?

These considerations led to the development of a new, quasi-local paradigm to describe black holes. This framework was inspired by certain seminal ideas introduced by Hayward [117, 118, $115,116]$ in the mid-nineties and has been systematically developed over the past five years or so. Evolving black holes are modelled by dynamical horizons while those in equilibrium are modelled by isolated horizons. Both notions are quasi-local. In contrast to event horizons, neither notion requires the knowledge of space-time as a whole or refers to asymptotic flatness. Furthermore, they are space-time notions. Therefore, in contrast to apparent horizons, they are not tied to the choice of a partial Cauchy slice. This framework provides a new perspective encompassing all areas in which black holes feature: quantum gravity, mathematical physics, numerical relativity, and gravitational wave phenomenology. Thus, it brings out the underlying unity of the subject. More importantly, it has overcome some of the limitations of the older frameworks and also led to new results of direct physical interest.

The purpose of this article is to review these developments. The subject is still evolving. Many of the key issues are still open and new results are likely to emerge in the coming years. Nonetheless, as the Editors pointed out, there is now a core of results of general interest and, thanks to the innovative style of Living Reviews, we will be able to incorporate new results through periodic updates.

Applications of the quasi-local framework can be summarized as follows:

\section{Black hole mechanics}

Isolated horizons extract from the notion of Killing horizons, just those conditions which ensure that the horizon geometry is time independent; there may be matter and radiation even nearby [68]. Yet, it has been possible to extend the zeroth and first laws of black hole mechanics to isolated horizons $[26,75,15]$. Furthermore, this derivation brings out a conceptually important fact about the first law. Recall that, in presence of internal boundaries, time evolution need not be Hamiltonian (i.e., need not preserve the symplectic structure). If the inner boundary is an isolated horizon, a necessary and sufficient condition for evolution to be Hamiltonian turns out to be precisely the first law! Finally, while the first law has the same form as before (Equation (1)), all quantities which enter the statement of the law now refer to the horizon itself. This is the case even when non-Abelian gauge fields are included.

Dynamical horizons allow for the horizon geometry to be time dependent. This framework has led to a quantitative relation between the growth of the horizon area and the flux of energy and angular momentum across it [30,31]. The processes can be in the non-linear regime of exact general relativity, without any approximations. Thus, the second law is generalized and the generalization also represents an integral version of the first law (1), applicable also when the black hole makes a transition from one state to another, which may be far removed.

\section{Quantum gravity}

The entropy problem refers to equilibrium situations. The isolated horizon framework provides an action principle and a Hamiltonian theory which serves as a stepping stone to non-perturbative quantization. Using the quantum geometry framework, a detailed theory of the quantum horizon geometry has been developed. The horizon states are then counted 
to show that the statistical mechanical black hole entropy is indeed proportional to the area $[10,11,84,149,25]$. This derivation is applicable to ordinary, astrophysical black holes which may be distorted and far from extremality. It also encompasses cosmological horizons to which thermodynamical considerations are known to apply [99]. Finally, the arena for this derivation is the curved black hole geometry, rather than a system in flat space-time which has the same number of states as the black hole $[174,145]$. Therefore, this approach has a greater potential for analyzing physical processes associated with the black hole.

The dynamical horizon framework has raised some intriguing questions about the relation between black hole mechanics and thermodynamics in fully dynamical situations [56]. In particular, they provide seeds for further investigations of the notion of entropy in nonequilibrium situations.

\section{Mathematical physics}

The isolated horizon framework has led to a phenomenological model to understand properties of hairy black holes [21, 20]. In this model, the hairy black hole can be regarded as a bound state of an ordinary black hole and a soliton. A large number of facts about hairy black holes had accumulated through semi-analytical and numerical studies. Their qualitative features are explained by the model.

The dynamical horizon framework also provides the groundwork for a new approach to Penrose inequalities which relate the area of cross-sections of the event horizon $A_{\mathrm{e}}$ on a Cauchy surface with the ADM mass $M_{\mathrm{ADM}}$ at infinity [157]: $\sqrt{A_{\mathrm{e}} / 16 \pi} \leq M_{\mathrm{ADM}}$. Relatively recently, the conjecture has been proved in time symmetric situations. The basic monotonicity formula of the dynamical horizon framework could provide a new avenue to extend the current proofs to non-time-symmetric situations. It may also lead to a stronger version of the conjecture where the ADM mass is replaced by the Bondi mass [31].

\section{Numerical relativity}

The framework has provided a number of tools to extract physics from numerical simulations in the near-horizon, strong field regime. First, there exist expressions for mass and angular momentum of dynamical and isolated horizons which enable one to monitor dynamical processes occurring in the simulations [31] and extract properties of the final equilibrium state $[15,85]$. These quantities can be calculated knowing only the horizon geometry and do not pre-suppose that the equilibrium state is a Kerr horizon. The computational resources required in these calculations are comparable to those employed by simulations using cruder techniques, but the results are now invariant and interpretation is free from ambiguities. Recent work [34] has shown that these methods are also numerically more accurate and robust than older ones.

Surprisingly, there are simple local criteria to decide whether the geometry of an isolated horizon is that of the Kerr horizon [142]. These criteria have already been implemented in numerical simulations. The isolated horizon framework also provides invariant, practical criteria to compare near-horizon geometries of different simulations [12] and leads to a new approach to the problem of extracting wave-forms in a gauge invariant fashion. Finally, the framework provides natural boundary conditions for the initial value problem for black holes in quasi-equilibrium [72, 124, 81], and to interpret certain initial data sets [136]. Many of these ideas have already been implemented in some binary black hole codes [85, 34, 48] and the process is continuing.

\section{Gravitational wave phenomenology}

The isolated horizon framework has led to a notion of horizon multipole moments [24]. They provide a diffeomorphism invariant characterization of the isolated horizon geometry. They

Living Reviews in Relativity

http: //www. livingreviews . org/Irr-2004-10 
are distinct from the Hansen multipoles in stationary space-times [107] normally used in the analysis of equations of motion because they depend only on the isolated horizon geometry and do not require global stationarity. They represent source multipoles rather than Hansen's field multipoles. In Kerr space-time, while the mass and angular momenta agree in the two regimes, quadrupole moments do not; the difference becomes significant when $a \sim M$, i.e., in the fully relativistic regime. In much of the literature on equations of motion of black holes, the distinction is glossed over largely because only field multipoles have been available in the literature. However, in applications to equations of motion, it is the source multipoles that are more relevant, whence the isolated horizon multipoles are likely to play a significant role.

The dynamical horizon framework enables one to calculate mass and angular momentum of the black hole as it evolves. In particular, one can now ask if the black hole can be first formed violating the Kerr bound $a \leq M$ but then eventually settle down in the Kerr regime. Preliminary considerations fail to rule out this possibility, although the issue is still open [31]. The issue can be explored both numerically and analytically. The possibility that the bound can indeed be violated initially has interesting astrophysical implications [89].

In this review, we will outline the basic ideas underlying dynamical and isolated horizon frameworks and summarize their applications listed above. The material is organized as follows. In Section 2 we recall the basic definitions, motivate the assumptions and summarize their implications. In Section 3 we discuss the area increase theorem for dynamical horizons and show how it naturally leads to an expression for the flux of gravitational energy crossing dynamical horizons. Section 4 is devoted to the laws of black hole mechanics. We outline the main ideas using both isolated and dynamical horizons. In the next three sections we review applications. Section 5 summarizes applications to numerical relativity, Section 6 to black holes with hair, and Section 7 to the quantum entropy calculation. Section 8 discusses open issues and directions for future work. Having read Section 2, Sections 3, 4, 5, 6, and 7 are fairly self contained and the three applications can be read independently of each other.

All manifolds will be assumed to be $C^{k+1}$ (with $k \geq 3$ ) and orientable, the space-time metric will be $C^{k}$, and matter fields $C^{k-2}$. For simplicity we will restrict ourselves to 4 -dimensional spacetime manifolds $\mathcal{M}$ (although most of the classical results on isolated horizons have been extended to 3-dimensions space-times [23], as well as higher dimensional ones [141]). The space-time metric $g_{a b}$ has signature $(-,+,+,+)$ and its derivative operator will be denoted by $\nabla$. The Riemann tensor is defined by $R_{a b c}{ }^{d} W_{d}:=2 \nabla_{[a} \nabla_{b]} W_{c}$, the Ricci tensor by $R_{a b}:=R_{a c b}{ }^{c}$, and the scalar curvature by $R:=g^{a b} R_{a b}$. We will assume the field equations

$$
R_{a b}-\frac{1}{2} R g_{a b}+\Lambda g_{a b}=8 \pi G T_{a b} .
$$

(With these conventions, de Sitter space-time has positive cosmological constant $\Lambda$.) We assume that $T_{a b}$ satisfies the dominant energy condition (although, as the reader can easily tell, several of the results will hold under weaker restrictions.) Cauchy (and partial Cauchy) surfaces will be denoted by $M$, isolated horizons by $\Delta$, and dynamical horizons by $H$. 


\section{Basic Notions}

This section is divided into two parts. The first introduces isolated horizons, and the second dynamical horizons.

\section{$2.1 \quad$ Isolated horizons}

In this part, we provide the basic definitions and discuss geometrical properties of non-expanding, weakly isolated, and isolated horizons which describe black holes which are in equilibrium in an increasingly stronger sense.

These horizons model black holes which are themselves in equilibrium, but in possibly dynamical space-times $[13,14,26,16]$. For early references with similar ideas, see $[156,106]$. A useful example is provided by the late stage of a gravitational collapse shown in Figure 1. In such physical situations, one expects the back-scattered radiation falling into the black hole to become negligible at late times so that the 'end portion' of the event horizon (labelled by $\Delta$ in the figure) can be regarded as isolated to an excellent approximation. This expectation is borne out in numerical simulations where the backscattering effects typically become smaller than the numerical errors rather quickly after the formation of the black hole (see, e.g., [34, 48]).

\subsubsection{Definitions}

The key idea is to extract from the notion of a Killing horizon the minimal conditions which are necessary to define physical quantities such as the mass and angular momentum of the black hole and to establish the zeroth and the first laws of black hole mechanics. Like Killing horizons, isolated horizons are null, 3-dimensional sub-manifolds of space-time. Let us therefore begin by recalling some essential features of such sub-manifolds, which we will denote by $\Delta$. The intrinsic metric $q_{a b}$ on $\Delta$ has signature $(0,+,+)$, and is simply the pull-back of the space-time metric to $\Delta$, $q_{a b}=g_{a b}$, where an underarrow indicates the pullback to $\Delta$. Since $q_{a b}$ is degenerate, it does not have an inverse in the standard sense. However, it does admit an inverse in a weaker sense: $q^{a b}$ will be said to be an inverse of $q_{a b}$ if it satisfies $q_{a m} q_{b n} q^{m n}=q_{a b}$. As one would expect, the inverse is not unique: We can always add to $q^{a b}$ a term of the type $\ell^{(a} V^{b)}$, where $\ell^{a}$ is a null normal to $\Delta$ and $V^{b}$ any vector field tangential to $\Delta$. All our constructions will be insensitive to this ambiguity. Given a null normal $\ell^{a}$ to $\Delta$, the expansion $\Theta_{(\ell)}$ is defined as

$$
\Theta_{(\ell)}:=q^{a b} \nabla_{a} \ell_{b} .
$$

(Throughout this review, we will assume that $\ell^{a}$ is future directed.) We can now state the first definition:

Definition 1: A sub-manifold $\Delta$ of a space-time $\left(\mathcal{M}, g_{a b}\right)$ is said to be a non-expanding horizon (NEH) if

1. $\Delta$ is topologically $S^{2} \times \mathbb{R}$ and null;

2. any null normal $\ell^{a}$ of $\Delta$ has vanishing expansion, $\Theta_{(\ell)}=0$; and

3. all equations of motion hold at $\Delta$ and the stress energy tensor $T_{a b}$ is such that $-T_{b}^{a} \ell^{b}$ is future-causal for any future directed null normal $\ell^{a}$.

The motivation behind this definition can be summarized as follows. Condition 1 is imposed for definiteness; while most geometric results are insensitive to topology, the $S^{2} \times \mathbb{R}$ case is physically the most relevant one. Condition 3 is satisfied by all classical matter fields of direct physical

Living Reviews in Relativity

http://www . livingreviews . org//rr-2004-10 
interest. The key condition in the above definition is Condition 2 which is equivalent to requiring that every cross-section of $\Delta$ be marginally trapped. (Note incidentally that if $\Theta_{(\ell)}$ vanishes for one null normal $\ell^{a}$ to $\Delta$, it vanishes for all.) Condition 2 is equivalent to requiring that the infinitesimal area element is Lie dragged by the null normal $\ell^{a}$. In particular, then, Condition 2 implies that the horizon area is 'constant in time'. We will denote the area of any cross section of $\Delta$ by $a_{\Delta}$ and define the horizon radius as $R_{\Delta}:=\sqrt{a_{\Delta} / 4 \pi}$.

Because of the Raychaudhuri equation, Condition 2 also implies

$$
R_{a b} \ell^{a} \ell^{b}+\sigma_{a b} \sigma^{a b}=0
$$

where $\sigma^{a b}$ is the shear of $\ell_{a}$, defined by $\sigma_{a b}:=\underline{\nabla}_{(a} \ell_{b)}-\frac{1}{2} \Theta_{(\ell)} q_{a b}$, where the underarrow denotes 'pull-back to $\Delta$ '. Now the energy condition 3 implies that $R_{a b} \ell^{a} \ell^{b}$ is non-negative, whence we conclude that each of the two terms in the last equation vanishes. This in turn implies that $T_{a b} \ell^{b}=0$ and $\nabla_{(a} \ell_{b)}=0$ on $\Delta$. The first of these equations constrains the matter fields on $\Delta$ in an interesting way, while the second is equivalent to $\mathcal{L}_{\ell} q_{a b}=0$ on $\Delta$. Thus, the intrinsic metric on an NEH is 'time-independent'; this is the sense in which an NEH is in equilibrium.

The zeroth and first laws of black hole mechanics require an additional structure, which is provided by the concept of a weakly isolated horizon. To arrive at this concept, let us first introduce a derivative operator $\mathcal{D}$ on $\Delta$. Because $q_{a b}$ is degenerate, there is an infinite number of (torsion-free) derivative operators which are compatible with it. However, on an NEH, the property $\underbrace{}_{(a} \ell_{b)}=0$ implies that the space-time connection $\nabla$ induces a unique (torsion-free) derivative operator $\mathcal{D}$ on $\Delta$ which is compatible with $q_{a b}[26,136]$. Weakly isolated horizons are characterized by the property that, in addition to the metric $q_{a b}$, the connection component $\mathcal{D}_{a} \ell^{b}$ is also 'time independent'.

Two null normals $\ell^{a}$ and $\tilde{\ell}^{a}$ to an NEH $\Delta$ are said to belong to the same equivalence class [ $\left.\ell\right]$ if $\tilde{\ell}^{a}=c \ell^{a}$ for some positive constant $c$. Then, weakly isolated horizons are defined as follows:

Definition 2: The pair $(\Delta,[\ell])$ is said to constitute a weakly isolated horizon (WIH) provided $\Delta$ is an NEH and each null normal $\ell^{a}$ in $[\ell]$ satisfies

$$
\left(\mathcal{L}_{\ell} \mathcal{D}_{a}-\mathcal{D}_{a} \mathcal{L}_{\ell}\right) \ell^{b}=0
$$

It is easy to verify that every NEH admits null normals satisfying Equation (5), i.e., can be made a WIH with a suitable choice of [ $\ell$. However the required equivalence class is not unique, whence an NEH admits distinct WIH structures [16].

Compared to conditions required of a Killing horizon, conditions in this definition are very weak. Nonetheless, it turns out that they are strong enough to capture the notion of a black hole in equilibrium in applications ranging from black hole mechanics to numerical relativity. (In fact, many of the basic notions such as the mass and angular momentum are well-defined already on NEHs although intermediate steps in derivations use a WIH structure.) This is quite surprising at first because the laws of black hole mechanics were traditionally proved for globally stationary black holes [182], and the definitions of mass and angular momentum of a black hole first used in numerical relativity implicitly assumed that the near horizon geometry is isometric to Kerr [5].

Although the notion of a WIH is sufficient for most applications, from a geometric viewpoint, a stronger notion of isolation is more natural: The full connection $\mathcal{D}$ should be time-independent. This leads to the notion of an isolated horizon.

Definition 3: $A W I H(\Delta,[\ell])$ is said to constitute an isolated horizon (IH) if

$$
\left(\mathcal{L}_{\ell} \mathcal{D}_{a}-\mathcal{D}_{a} \mathcal{L}_{\ell}\right) V^{b}=0
$$


for arbitrary vector fields $V^{a}$ tangential to $\Delta$.

While an NEH can always be given a WIH structure simply by making a suitable choice of the null normal, not every WIH admits an IH structure. Thus, the passage from a WIH to an $\mathrm{IH}$ is a genuine restriction [16]. However, even for this stronger notion of isolation, conditions in the definition are local to $\Delta$. Furthermore, the definition only uses quantities intrinsic to $\Delta$; there are no restrictions on components of any fields transverse to $\Delta$. (Even the full 4-metric $g_{a b}$ need not be time independent on the horizon.) Robinson-Trautman solutions provide explicit examples of isolated horizons which do not admit a stationary Killing field even in an arbitrarily small neighborhood of the horizon [68]. In this sense, the conditions in this definition are also rather weak. One expects them to be met to an excellent degree of approximation in a wide variety of situations representing late stages of gravitational collapse and black hole mergers ${ }^{2}$.

\subsubsection{Examples}

The class of space-times admitting NEHs, WIHs, and IHs is quite rich. First, it is trivial to verify that any Killing horizon which is topologically $S^{2} \times \mathbb{R}$ is also an isolated horizon. This in particular implies that the event horizons of all globally stationary black holes, such as the Kerr-Newman solutions (including a possible cosmological constant), are isolated horizons. (For more exotic examples, see [155].)

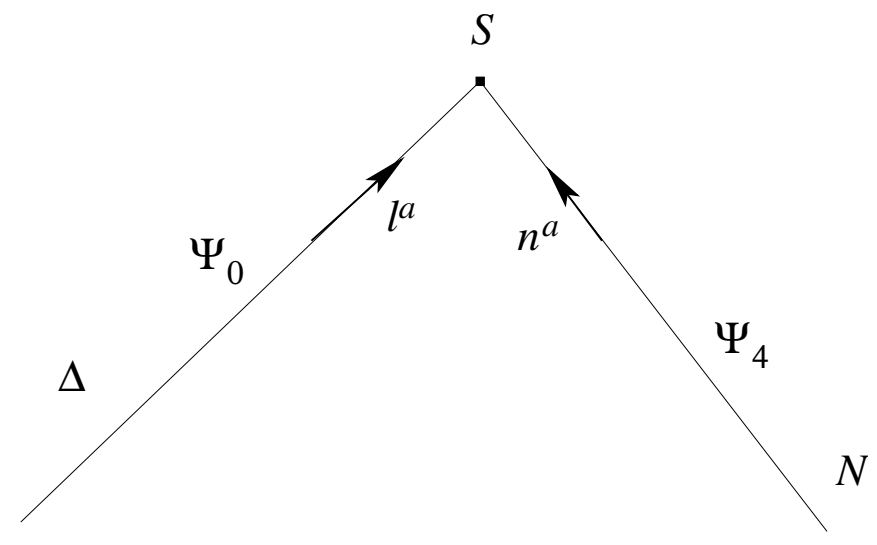

Figure 3: Set-up of the general characteristic initial value formulation. The Weyl tensor component $\Psi_{0}$ on the null surface $\Delta$ is part of the free data which vanishes if $\Delta$ is an IH.

But there exist other non-trivial examples as well. These arise because the notion is quasi-local, referring only to fields defined intrinsically on the horizon. First, let us consider the sub-family of Kastor-Traschen solutions [125, 152] which are asymptotically de Sitter and admit event horizons. They are interpreted as containing multiple charged, dynamical black holes in presence of a positive cosmological constant. Since these solutions do not appear to admit any stationary Killing fields, no Killing horizons are known to exist. Nonetheless, the event horizons of individual black holes are WIHs. However, to our knowledge, no one has checked if they are IHs. A more striking example is provided by a sub-family of Robinson-Trautman solutions, analyzed by Chrusciel [68]. These space-times admit IHs whose intrinsic geometry is isomorphic to that of the Schwarzschild isolated horizons but in which there is radiation arbitrarily close to $\Delta$.

\footnotetext{
${ }^{2}$ However, Condition (6) may be too strong in some problems, e.g., in the construction of quasi-equilibrium initial data sets, where the notion of WIH is more useful [124] (see Section 5.2).
}

Living Reviews in Relativity

http: //www. livingreviews . org/Irr-2004-10 
More generally, using the characteristic initial value formulation [92, 161], Lewandowski [140] has constructed an infinite dimensional set of local examples. Here, one considers two null surfaces $\Delta$ and $\mathcal{N}$ intersecting in a 2 -sphere $S$ (see Figure 3). One can freely specify certain data on these two surfaces which then determines a solution to the vacuum Einstein equations in a neighborhood of $S$ bounded by $\Delta$ and $\mathcal{N}$, in which $\Delta$ is an isolated horizon.

\subsubsection{Geometrical properties}

\section{Rescaling freedom in $\ell^{a}$}

As we remarked in Section 2.1.1, there is a functional rescaling freedom in the choice of a null normal on an NEH and, while the choice of null normals is restricted by the weakly isolated horizon condition (5), considerable freedom still remains. That is, a given NEH $\Delta$ admits an infinite number of WIH structures $(\Delta,[\ell])[16]$.

On IHs, by contrast, the situation is dramatically different. Given an $\mathrm{IH}(\Delta,[\ell])$, generically the Condition (6) in Definition 3 can not be satisfied by a distinct equivalence class of null normals $\left[\ell^{\prime}\right]$. Thus on a generic IH, the only freedom in the choice of the null normal is that of a rescaling by a positive constant [16]. This freedom mimics the properties of a Killing horizon since one can also rescale the Killing vector by an arbitrary constant. The triplet $\left(q_{a b}, \mathcal{D}_{a},\left[\ell^{a}\right]\right)$ is said to constitute the geometry of the isolated horizon.

\section{Surface gravity}

Let us begin by defining a 1-form $\omega_{a}$ which will be used repeatedly. First note that, by Definition $1, \ell^{a}$ is expansion free and shear free. It is automatically twist free since it is a normal to a smooth hypersurface. This means that the contraction of $\nabla_{a} \ell_{b}$ with any two vectors tangent to $\Delta$ is identically zero, whence there must exist a 1 -form $\omega_{a}$ on $\Delta$ such that for any $V^{a}$ tangent to $\Delta$,

$$
V^{a} \nabla_{a} \ell^{b}=V^{a} \omega_{a} \ell^{b} .
$$

Note that the WIH condition (5) requires simply that $\omega_{a}$ be time independent, $\mathcal{L}_{\ell} \omega_{a}=0$. Given $\omega_{a}$, the surface gravity $\kappa_{(\ell)}$ associated with a null normal $\ell^{a}$ is defined as

$$
\kappa_{(\ell)}:=\ell^{a} \omega_{a} .
$$

Thus, $\kappa_{(\ell)}$ is simply the acceleration of $\ell^{a}$. Note that the surface gravity is not an intrinsic property of a WIH $(\Delta,[\ell])$. Rather, it is a property of a null normal to $\Delta: \kappa_{(c \ell)}=c \kappa_{(\ell)}$. An isolated horizon with $\kappa_{(\ell)}=0$ is said to be an extremal isolated horizon. Note that while the value of surface gravity refers to a specific null normal, whether a given WIH is extremal or not is insensitive to the permissible rescaling of the normal.

\section{Curvature tensors on $\Delta$}

Consider any (space-time) null tetrad $\left(\ell^{a}, n^{a}, m^{a}, \bar{m}^{a}\right)$ on $\Delta$ such that $\ell^{a}$ is a null normal to $\Delta$. Then, it follows from Definition 1 that two of the Newman-Penrose Weyl components vanish on $\Delta: \Psi_{0}:=C_{a b c d} \ell^{a} m^{b} \ell^{c} m^{d}=0$ and $\Psi_{1}:=C_{a b c d} \ell^{a} m^{b} \ell^{c} n^{d}=0$. This in turn implies that $\Psi_{2}:=C_{a b c d} \ell^{a} m^{b} \bar{m}^{c} n^{d}$ is gauge invariant (i.e., does not depend on the specific choice of the null tetrad satisfying the condition stated above.) The imaginary part of $\Psi_{2}$ is related to the curl of $\omega_{a}$,

$$
d \omega=2\left(\operatorname{Im} \Psi_{2}\right) \epsilon,
$$

where $\epsilon_{a b}$ is the natural area 2-form on $\Delta$. Horizons on which $\operatorname{Im} \Psi_{2}$ vanishes are said to be non-rotating: On these horizons all angular momentum multipoles vanish [24]. Therefore, $\operatorname{Im} \Psi_{2}$ is sometimes referred to as the rotational scalar and $\omega_{a}$ as the rotation 1-form of the horizon. 
Next, let us consider the Ricci-tensor components. On any NEH $\Delta$ we have: $\Phi_{00}:=$ $\frac{1}{2} R_{a b} \ell^{a} \ell^{b}:=0, \Phi_{01}:=\frac{1}{2} R_{a b} \ell^{a} m^{b}=0$. In the Einstein-Maxwell theory, one further has: On $\Delta, \Phi_{02}:=\frac{1}{2} R_{a b} m^{a} m^{b}=0$ and $\Phi_{20}:=\frac{1}{2} R_{a b} \bar{m}^{a} \bar{m}^{b}=0$.

\section{Free data on an isolated horizon}

Given the geometry $\left(q_{a b}, \mathcal{D},[\ell]\right)$ of an $\mathrm{IH}$, it is natural to ask for the minimum amount of information, i.e., the free data, required to construct it. This question has been answered in detail (also for WIHs) [16]. For simplicity, here we will summarize the results only for the non-extremal case. (For the extremal case, see [16, 143].)

Let $S$ be a spherical cross section of $\Delta$. The degenerate metric $q_{a b}$ naturally projects to a Riemannian metric $\tilde{q}_{a b}$ on $S$, and similarly the 1-form $\omega_{a}$ of Equation (7) projects to a 1 -form $\tilde{\omega}_{a}$ on $S$. If the vacuum equations hold on $\Delta$, then given $\left(\tilde{q}_{a b}, \tilde{\omega}_{a}\right)$ on $S$, there is, up to diffeomorphisms, a unique non-extremal isolated horizon geometry $\left(q_{a b}, \mathcal{D},[\ell]\right)$ such that $\tilde{q}_{a b}$ is the projection of $q_{a b}, \tilde{\omega}_{a}$ is the projection of the $\omega_{a}$ constructed from $\mathcal{D}$, and $\kappa_{(\ell)}=\omega_{a} \ell^{a} \neq 0$. (If the vacuum equations do not hold, the additional data required is the projection on $S$ of the space-time Ricci tensor.)

The underlying reason behind this result can be sketched as follows. First, since $q_{a b}$ is degenerate along $\ell^{a}$, its non-trivial part is just its projection $\tilde{q}_{a b}$. Second, $\tilde{q}_{a b}$ fixes the connection on $S$; it is only the quantity $S_{a b}:=\mathcal{D}_{a} n_{b}$ that is not constrained by $\tilde{q}_{a b}$, where $n_{a}$ is a 1 -form on $\Delta$ orthogonal to $S$, normalized so that $\ell^{a} n_{a}=-1$. It is easy to show that $S_{a b}$ is symmetric and the contraction of one of its indices with $\ell^{a}$ gives $\omega_{a}: \ell^{a} S_{a b}=\omega_{a}$. Furthermore, it turns out that if $\omega_{a} \ell^{a} \neq 0$, the field equations completely determine the angular part of $S_{a b}$ in terms of $\tilde{\omega}_{a}$ and $\tilde{q}_{a b}$. Finally, recall that the surface gravity is not fixed on $\Delta$ because of the rescaling freedom in $\ell^{a}$; thus the $\ell$-component of $\omega_{a}$ is not part of the free data. Putting all these facts together, we see that the pair $\left(\tilde{q}_{a b}, \tilde{\omega}_{a}\right)$ enables us to reconstruct the isolated horizon geometry uniquely up to diffeomorphisms.

\section{Rest frame of a non-expanding horizon}

As at null infinity, a preferred foliation of $\Delta$ can be thought of as providing a 'rest frame' for an isolated horizon. On the Schwarzschild horizon, for example, the 2-spheres on which the Eddington-Finkelstein advanced time coordinate is constant - which are also integral manifolds of the rotational Killing fields - provide such a rest frame. For the Kerr metric, this foliation generalizes naturally. The question is whether a general prescription exists to select such a preferred foliation.

On any non-extremal NEH, the 1-form $\omega_{a}$ can be used to construct preferred foliations of $\Delta$. Let us first examine the simpler, non-rotating case in which $\operatorname{Im} \Psi_{2}=0$. Then Equation (9) implies that $\omega_{a}$ is curl-free and therefore hypersurface orthogonal. The 2-surfaces orthogonal to $\omega_{a}$ must be topologically $S^{2}$ because, on any non-extremal horizon, $\ell^{a} \omega_{a} \neq 0$. Thus, in the non-rotating case, every isolated horizon comes equipped with a preferred family of cross-sections which defines the rest frame [26]. Note that the projection $\tilde{\omega}_{a}$ of $\omega_{a}$ on any leaf of this foliation vanishes identically.

The rotating case is a little more complicated since $\omega_{a}$ is then no longer curl-free. Now the idea is to exploit the fact that the divergence of the projection $\tilde{\omega}_{a}$ of $\omega_{a}$ on a cross-section is sensitive to the choice of the cross-section, and to select a preferred family of cross-sections by imposing a suitable condition on this divergence [16]. A mathematically natural choice is to ask that this divergence vanish. However, (in the case when the angular momentum is non-zero) this condition does not pick out the $v=$ const. cuts of the Kerr horizon where $v$ is the (Carter generalization of the) Eddington-Finkelstein coordinate. Pawlowski has provided another condition that also selects a preferred foliation and reduces to the $v=$ const. cuts of

Living Reviews in Relativity

http://www . livingreviews . org//rr-2004-10 
the Kerr horizon:

$$
\operatorname{div} \tilde{\omega}=-\tilde{\Delta} \ln \left|\Psi_{2}\right|^{1 / 3}
$$

where $\tilde{\Delta}$ is the Laplacian of $\tilde{q}_{a b}$. On isolated horizons on which $\left|\Psi_{2}\right|$ is nowhere zero - a condition satisfied if the horizon geometry is 'near' that of the Kerr isolated horizon - this selects a preferred foliation and hence a rest frame. This construction is potentially useful to numerical relativity.

\section{Symmetries of an isolated horizon}

By definition, a symmetry of an IH $(\Delta,[\ell])$ is a diffeomorphism of $\Delta$ which preserves the geometry $\left(q_{a b}, \mathcal{D},[\ell]\right)$. (On a WIH, the symmetry has to preserve $\left(q_{a b}, \omega_{a},[\ell]\right)$. There are again three universality classes of symmetry groups as on an IH.) Let us denote the symmetry group by $G_{\Delta}$. First note that diffeomorphisms generated by the null normals in $\left[\ell^{a}\right]$ are symmetries; this is already built into the very definition of an isolated horizon. The other possible symmetries are related to the cross-sections of $\Delta$. Since we have assumed the crosssections to be topologically spherical and since a metric on a sphere can have either exactly three, one or zero Killing vectors, it follows that $G_{\Delta}$ can be of only three types [15]:

- Type I: The pair $\left(q_{a b}, \mathcal{D}_{a}\right)$ is spherically symmetric; $G_{\Delta}$ is four dimensional.

- Type II: The pair $\left(q_{a b}, \mathcal{D}_{a}\right)$ is axisymmetric; $G_{\Delta}$ is two dimensional.

- Type III: Diffeomorphisms generated by $\ell^{a}$ are the only symmetries of the pair $\left(q_{a b}, \mathcal{D}_{a}\right)$; $G_{\Delta}$ is one dimensional.

In the asymptotically flat context, boundary conditions select a universal symmetry group at spatial infinity, e.g., the Poincaré group, because the space-time metric approaches a fixed Minkowskian one. The situation is completely different in the strong field region near a black hole. Because the geometry at the horizon can vary from one space-time to another, the symmetry group is not universal. However, the above result shows that the symmetry group can be one of only three universality classes.

\subsection{Dynamical horizons}

This section is divided into three parts. In the first, we discuss basic definitions, in the second we introduce an explicit example, and in the third we analyze the issue of uniqueness of dynamical horizons and their role in numerical relativity.

\subsubsection{Definitions}

To explain the evolution of ideas and provide points of comparison, we will introduce the notion of dynamical horizons following a chronological order. Readers who are not familiar with causal structures can go directly to Definition 5 of dynamical horizons (for which a more direct motivation can be found in [31]).

As discussed in Section 1, while the notion of an event horizon has proved to be very convenient in mathematical relativity, it is too global and teleological to be directly useful in a number of physical contexts ranging from quantum gravity to numerical relativity to astrophysics. This limitation was recognized early on (see, e.g., [113], page 319) and alternate notions were introduced to capture the intuitive idea of a black hole in a quasi-local manner. In particular, to make the concept 'local in time', Hawking [111, 113] introduced the notions of a trapped region and an apparent horizon, both of which are associated to a space-like 3-surface $M$ representing 'an instant of time'. Let us begin by recalling these ideas. 
Hawking's outer trapped surface $S$ is a compact, space-like 2-dimensional sub-manifold in $\left(\mathcal{M}, g_{a b}\right)$ such that the expansion $\Theta_{(\ell)}$ of the outgoing null normal $\ell^{a}$ to $S$ is non-positive. Hawking then defined the trapped region $\mathcal{T}(M)$ in a surface $M$ as the set of all points in $M$ through which there passes an outer-trapped surface, lying entirely in $M$. Finally, Hawking's apparent horizon $\partial \mathcal{T}(M)$ is the boundary of a connected component of $\mathcal{T}(M)$. The idea then was to regard each apparent horizon as the instantaneous surface of a black hole. One can calculate the expansion $\Theta_{(\ell)}$ of $S$ knowing only the intrinsic 3-metric $q_{a b}$ and the extrinsic curvature $K_{a b}$ of $M$. Hence, to find outer trapped surfaces and apparent horizons on $M$, one does not need to evolve $\left(q_{a b}, K_{a b}\right)$ away from $M$ even locally. In this sense the notion is local to $M$. However, this locality is achieved at the price of restricting $S$ to lie in $M$. If we wiggle $M$ even slightly, new outer trapped surfaces can appear and older ones may disappear. In this sense, the notion is still very global. Initially, it was hoped that the laws of black hole mechanics can be extended to these apparent horizons. However, this has not been possible because the notion is so sensitive to the choice of $M$.

To improve on this situation, in the early nineties Hayward proposed a novel modification of this framework [117]. The main idea is to free these notions from the complicated dependence on $M$. He began with Penrose's notion of a trapped surface. A trapped surface $S$ a la Penrose is a compact, space-like 2-dimensional sub-manifold of space-time on which $\Theta_{(\ell)} \Theta_{(n)}>0$, where $\ell^{a}$ and $n^{a}$ are the two null normals to $S$. We will focus on future trapped surfaces on which both expansions are negative. Hayward then defined a space-time trapped region. A trapped region $\mathcal{T}$ a la Hayward is a subset of space-time through each point of which there passes a trapped surface. Finally, Hayward's trapping boundary $\partial \mathcal{T}$ is a connected component of the boundary of an inextendible trapped region. Under certain assumptions (which appear to be natural intuitively but technically are quite strong), he was able to show that the trapping boundary is foliated by marginally trapped surfaces (MTSs), i.e., compact, space-like 2-dimensional sub-manifolds on which the expansion of one of the null normals, say $\ell^{a}$, vanishes and that of the other, say $n^{a}$, is everywhere non-positive. Furthermore, $\mathcal{L}_{n} \Theta_{(\ell)}$ is also everywhere of one sign. These general considerations led him to define a quasi-local analog of future event horizons as follows:

Definition 4: A future, outer, trapping horizon (FOTH) is a smooth 3-dimensional sub-manifold $\underline{H}$ of space-time, foliated by closed 2-manifolds $\underline{S}$, such that

1. the expansion of one future directed null normal to the foliation, say $\ell^{a}$, vanishes, $\Theta_{(\ell)}=0$;

2. the expansion of the other future directed null normal $n^{a}$ is negative, $\Theta_{(n)}<0$; and

3. the directional derivative of $\Theta_{(\ell)}$ along $n^{a}$ is negative, $\mathcal{L}_{n} \Theta_{(\ell)}<0$.

In this definition, Condition 2 captures the idea that $H$ is a future horizon (i.e., of black hole rather than white hole type), and Condition 3 encodes the idea that it is 'outer' since infinitesimal motions along the 'inward' normal $n^{a}$ makes the 2-surface trapped. (Condition 3 also serves to distinguish black hole type horizons from certain cosmological ones [117] which are not ruled out by Condition 2). Using the Raychaudhuri equation, it is easy to show that $\underline{H}$ is either space-like or null, being null if and only if the shear $\sigma_{a b}$ of $\ell^{a}$ as well as the matter flux $T_{a b} \ell^{a} \ell^{b}$ across $H$ vanishes. Thus, when $\underline{H}$ is null, it is a non-expanding horizon introduced in Section 2.1. Intuitively, $\underline{H}$ is space-like in the dynamical region where gravitational radiation and matter fields are pouring into it and is null when it has reached equilibrium.

In truly dynamical situations, then, $\underline{H}$ is expected to be space-like. Furthermore, it turns out that most of the key results of physical interest [30, 31], such as the area increase law and generalization of black hole mechanics, do not require the condition on the sign of $\mathcal{L}_{n} \Theta_{(\ell)}$. It is therefore convenient to introduce a simpler and at the same time 'tighter' notion, that of a dynamical horizon, which is better suited to analyze how black holes grow in exact general relativity [30, 31]:

Definition 5: A smooth, three-dimensional, space-like sub-manifold (possibly with boundary) $H$

Living Reviews in Relativity

http://www . livingreviews . org//rr-2004-10 
of space-time is said to be a dynamical horizon (DH) if it can be foliated by a family of closed 2-manifolds such that

1. on each leaf $S$ the expansion $\Theta_{(\ell)}$ of one null normal $\ell^{a}$ vanishes; and

2. the expansion $\Theta_{(n)}$ of the other null normal $n^{a}$ is negative.

Note first that, like FOTHs, dynamical horizons are 'space-time notions', defined quasi-locally. They are not defined relative to a space-like surface as was the case with Hawking's apparent horizons nor do they make any reference to infinity as is the case with event horizons. In particular, they are well-defined also in the spatially compact context. Being quasi-local, they are not teleological. Next, let us spell out the relation between FOTHs and DHs. A space-like FOTH is a DH on which the additional condition $\mathcal{L}_{n} \Theta_{(\ell)}<0$ holds. Similarly, a DH satisfying $\mathcal{L}_{n} \Theta_{(\ell)}<0$ is a space-like FOTH. Thus, while neither definition implies the other, the two are closely related. The advantage of Definition 5 is that it refers only to the intrinsic structure of $H$, without any conditions on the evolution of fields in directions transverse to $H$. Therefore, it is easier to verify in numerical simulations. More importantly, as we will see, this feature makes it natural to analyze the structure of $H$ using only the constraint (or initial value) equations on it. This analysis will lead to a wealth of information on black hole dynamics. Reciprocally, Definition 4 has the advantage that, since it permits $\underline{H}$ to be space-like or null, it is better suited to analyze the transition to equilibrium [31].

A DH which is also a FOTH will be referred to as a space-like future outer horizon (SFOTH). To fully capture the physical notion of a dynamical black hole, one should require both sets of conditions, i.e., restrict oneself to SFOTHs. For, stationary black holes admit FOTHS and there exist space-times [166] which admit dynamical horizons but no trapped surfaces; neither can be regarded as containing a dynamical black hole. However, it is important to keep track of precisely which assumptions are needed to establish specific results. Most of the results reported in this review require only those conditions which are satisfied on DHs. This fact may well play a role in conceptual issues that arise while generalizing black hole thermodynamics to non-equilibrium situations ${ }^{3}$.

\subsubsection{Examples}

Let us begin with the simplest examples of space-times admitting DHs (and SFOTHs). These are provided by the spherically symmetric solution to Einstein's equations with a null fluid as source, the Vaidya metric $[179,137,186]$. (Further details and the inclusion of a cosmological constant are discussed in [31].) Just as the Schwarzschild-Kruskal solution provides a great deal of intuition for general static black holes, the Vaidya metric furnishes some of the much needed intuition in the dynamical regime by bringing out the key differences between the static and dynamical situations. However, one should bear in mind that both Schwarzschild and Vaidya black holes are the simplest examples and certain aspects of geometry can be much more complicated in more general situations. The 4-metric of the Vaidya space-time is given by

$$
g_{a b}=-\left(1-\frac{2 G M(v)}{r}\right) \nabla_{a} v \nabla_{b} v+2 \nabla_{(a} v \nabla_{b)} r+r^{2}\left(\nabla_{a} \theta \nabla_{b} \theta+\sin ^{2} \theta \nabla_{a} \phi \nabla_{b} \phi\right),
$$

where $M(v)$ is any smooth, non-decreasing function of $v$. Thus, $(v, r, \theta, \phi)$ are the ingoing EddingtonFinkelstein coordinates. This is a solution of Einstein's equations, the stress-energy tensor $T_{a b}$ being

\footnotetext{
${ }^{3}$ Indeed, the situation is similar for black holes in equilibrium. While it is physically reasonable to restrict oneself to IHs, most results require only the WIH boundary conditions. The distinction can be important in certain applications, e.g., in finding boundary conditions on the quasi-equilibrium initial data at inner horizons.
} 
given by

$$
T_{a b}=\frac{\dot{M}(v)}{4 \pi r^{2}} \nabla_{a} v \nabla_{b} v,
$$

where $\dot{M}=d M / d v$. Clearly, $T_{a b}$ satisfies the dominant energy condition if $\dot{M} \geq 0$, and vanishes if and only if $\dot{M}=0$. Of special interest to us are the cases illustrated in Figure $4: M(v)$ is non-zero until a certain finite retarded time, say $v=0$, and then grows monotonically, either reaching an asymptotic value $M_{0}$ as $v$ tends to infinity (panel a), or, reaching this value at a finite retarded time, say $v=v_{0}$, and then remaining constant (panel b). In either case, the space-time region $v \leq 0$ is flat.

Let us focus our attention on the metric 2-spheres, which are all given by $v=$ const. and $r=$ const.. It is easy to verify that the expansion of the outgoing null normal $\ell^{a}$ vanishes if and only if $(v=$ const. and) $r=2 G M(v)$. Thus, these are the only spherically symmetric marginally trapped surfaces MTSs. On each of them, the expansion $\Theta_{(n)}$ of the ingoing normal $n^{a}$ is negative. By inspection, the 3-metric on the world tube $r=2 G M(v)$ of these MTSs has signature $(+,+,+)$ when $\dot{M}(v)$ is non-zero and $(0,+,+)$ if $\dot{M}(v)$ is zero. Hence, in the left panel of Figure 4 the surface $r=2 G M(v)$ is the $\mathrm{DH} H$. In the right panel of Figure 4 the portion of this surface $v \leq v_{0}$ is the $\mathrm{DH} H$, while the portion $v \geq v_{0}$ is a non-expanding horizon. (The general issue of transition of a $\mathrm{DH}$ to equilibrium is briefly discussed in Section 5.) Finally, note that at these MTSs, $\mathcal{L}_{n} \theta_{(\ell)}=-2 / r^{2}<0$. Hence in both cases, the DH is an SFOTH. Furthermore, in the case depicted in the right panel of Figure 4 the entire surface $r=2 G M(v)$ is a FOTH, part of which is dynamical and part null.

This simple example also illustrates some interesting features which are absent in the stationary situations. First, by making explicit choices of $M(v)$, one can plot the event horizon using, say, Mathematica [189] and show that they originate in the flat space-time region $v<0$, in anticipation of the null fluid that is going to fall in after $v=0$. The dynamical horizon, on the other hand, originates in the curved region of space-time, where the metric is time-dependent, and steadily expands until it reaches equilibrium. Finally, as Figures 4 illustrate, the dynamical and event horizons can be well separated. Recall that in the equilibrium situation depicted by the Schwarzschild space-time, a spherically symmetric trapped surface passes through every point in the interior of the event horizon. In the dynamical situation depicted by the Vaidya space-time, they all lie in the interior of the DH. However, in both cases, the event horizon is the boundary of $J^{-}\left(\mathcal{I}^{+}\right)$. Thus, the numerous roles played by the event horizon in equilibrium situations get split in dynamical contexts, some taken up by the $\mathrm{DH}$.

What is the situation in a more general gravitational collapse? As indicated in the beginning of this section, the geometric structure can be much more subtle. Consider 3-manifolds $\tau$ which are foliated by marginally trapped compact 2 -surfaces $S$. We denote by $\ell^{a}$ the normal whose expansion vanishes. If the expansion of the other null normal $n^{a}$ is negative, $\tau$ will be called a marginally trapped tube (MTT). If the tube $\tau$ is space-like, it is a dynamical horizon. If it is time-like, it will be called time-like membrane. Since future directed causal curves can traverse time-like membranes in either direction, they are not good candidates to represent surfaces of black holes; therefore they are not referred to as horizons.

In Vaidya metrics, there is precisely one MTT to which all three rotational Killing fields are tangential and this is the DH $H$. In the Oppenheimer-Volkoff dust collapse, however, the situation is just the opposite; the unique MTT on which each MTS $S$ is spherical is time-like [180, 47]. Thus we have a time-like membrane rather than a dynamical horizon. However, in this case the metric does not satisfy the smoothness conditions spelled out at the end of Section 1 and the global timelike character of $\tau$ is an artifact of the lack of this smoothness. In the general perfect fluid spherical collapse, if the solution is smooth, one can show analytically that the spherical MTT is space-like at sufficiently late times, i.e., in a neighborhood of its intersection with the event horizon [102]. For the spherical scalar field collapse, numerical simulations show that, as in the Vaidya solutions,

Living Reviews in Relativity

http://www . livingreviews . org//rr-2004-10 

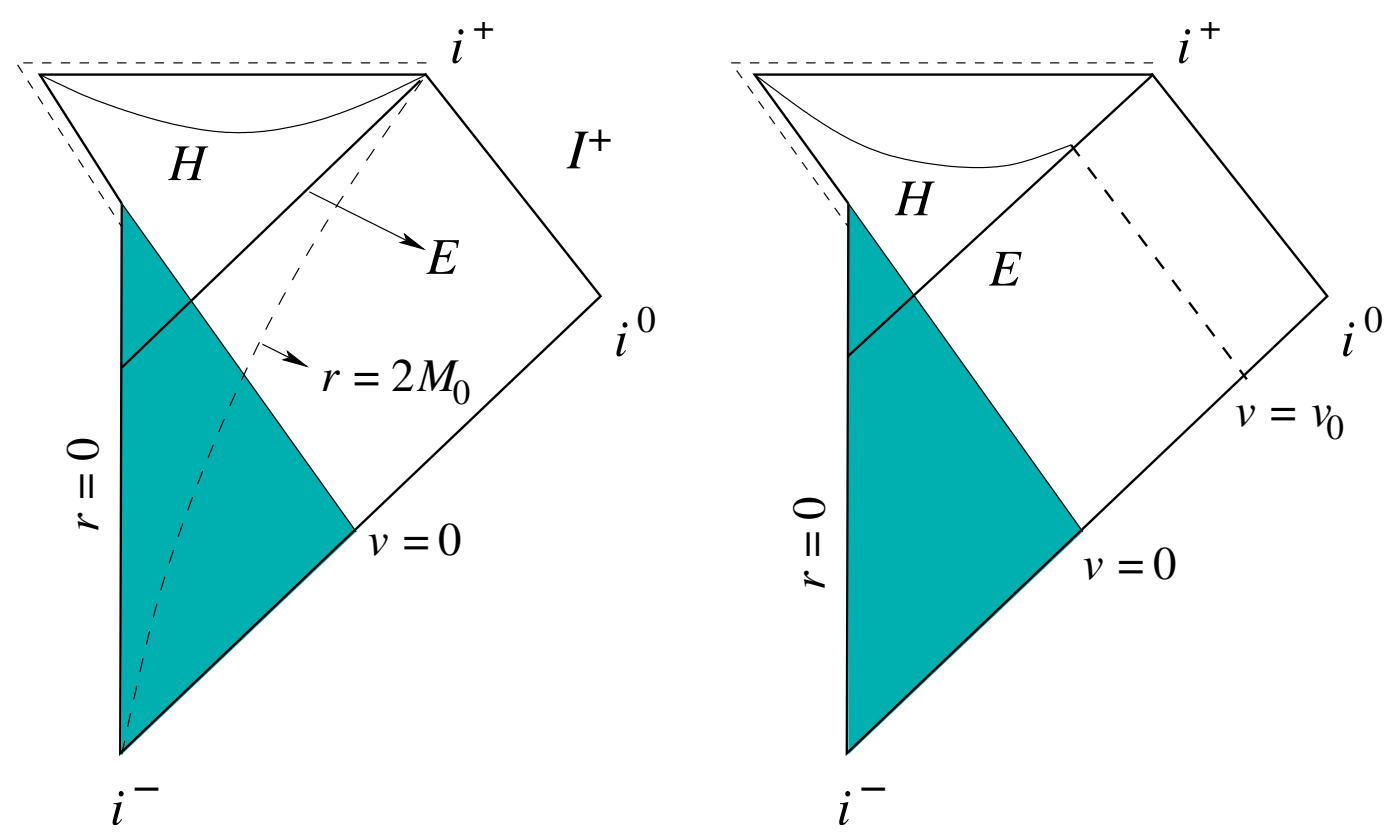

Figure 4: Penrose diagrams of Schwarzschild-Vaidya metrics for which the mass function $M(v)$ vanishes for $v \leq 0$ [137]. The space-time metric is flat in the past of $v=0$ (i.e., in the shaded region). In the left panel, as $v$ tends to infinity, $\dot{M}$ vanishes and $M$ tends to a constant value $M_{0}$. The space-like dynamical horizon $H$, the null event horizon $E$, and the time-like surface $r=2 M_{0}$ (represented by the dashed line) all meet tangentially at $i^{+}$. In the right panel, for $v \geq v_{0}$ we have $\dot{M}=0$. Space-time in the future of $v=v_{0}$ is isometric with a portion of the Schwarzschild space-time. The dynamical horizon $H$ and the event horizon $E$ meet tangentially at $v=v_{0}$. In both figures, the event horizon originates in the shaded flat region, while the dynamical horizon exists only in the curved region. 
Abhay Ashtekar and Badri Krishnan

the spherical MTT is space-like everywhere [102]. Finally, the geometry of the numerically evolved MTTs has been examined in two types of non-spherical situations: the axi-symmetric collapse of a neutron star to a Kerr black hole and in the head-on collision of two non-rotating black holes [48]. In both cases, in the initial phase the MTT is neither space-like nor time-like all the way around its cross-sections $S$. However, it quickly becomes space-like and has a long space-like portion which approaches the event horizon. This portion is then a dynamical horizon. There are no hard results on what would happen in general, physically interesting situations. The current expectation is that the MTT of a numerically evolved black hole space-time which asymptotically approaches the event horizon will become space-like rather soon after its formation. Therefore most of the ongoing detailed work focuses on this portion, although basic analytical results are available also on how the time-like membranes evolve (see Appendix A of [31]).

\subsubsection{Uniqueness}

Even in the simplest, Vaidya example discussed above, our explicit calculations were restricted to spherically symmetric marginally trapped surfaces. Indeed, already in the case of the Schwarzschild space-time, very little is known analytically about non-spherically symmetric marginally trapped surfaces. It is then natural to ask if the Vaidya metric admits other, non-spherical dynamical horizons which also asymptote to the non-expanding one. Indeed, even if we restrict ourselves to the 3-manifold $r=2 G M(v)$, can we find another foliation by non-spherical, marginally trapped surfaces which endows it with another dynamical horizon structure? These considerations illustrate that in general there are two uniqueness issues that must be addressed.

First, in a general space-time $\left(\mathcal{M}, g_{a b}\right)$, can a space-like 3 -manifold $H$ be foliated by two distinct families of marginally trapped surfaces, each endowing it with the structure of a dynamical horizon? Using the maximum principle, one can show that this is not possible [93]. Thus, if $H$ admits a dynamical horizon structure, it is unique.

Second, we can ask the following question: How many DHs can a space-time admit? Since a space-time may contain several distinct black holes, there may well be several distinct DHs. The relevant question is if distinct DHs can exist within each connected component of the (spacetime) trapped region. On this issue there are several technically different uniqueness results [27]. It is simplest to summarize them in terms of SFOTHs. First, if two non-intersecting SFOTHs $H$ and $H^{\prime}$ become tangential to the same non-expanding horizon at a finite time (see the right panel in Figure 4), then they coincide (or one is contained in the other). Physically, a more interesting possibility, associated with the late stages of collapse or mergers, is that $H$ and $H^{\prime}$ become asymptotic to the event horizon. Again, they must coincide in this case. At present, one can not rule out the existence of more than one SFOTHs which asymptote to the event horizon if they intersect each other repeatedly. However, even if this were to occur, the two horizon geometries would be non-trivially constrained. In particular, none of the marginally trapped surfaces on $H$ can lie entirely to the past of $H^{\prime}$.

A better control on uniqueness is perhaps the most important open issue in the basic framework for dynamical horizons and there is ongoing work to improve the existing results. Note however that all results of Sections 3 and 5, including the area increase law and the generalization of black hole mechanics, apply to all DHs (including the 'transient ones' which may not asymptote to the event horizon). This makes the framework much more useful in practice.

The existing results also provide some new insights for numerical relativity [27]. First, suppose that a MTT $\tau$ is generated by a foliation of a region of space-time by partial Cauchy surfaces $M_{t}$ such that each MTS $S_{t}$ is the outermost MTS in $M_{t}$. Then $\tau$ can not be a time-like membrane. Note however that this does not imply that $\tau$ is necessarily a dynamical horizon because $\tau$ may be partially time-like and partially space-like on each of its marginally trapped surfaces $S$. The requirement that $\tau$ be space-like - i.e., be a dynamical horizon - would restrict the choice of the

Living Reviews in Relativity

http://www . livingreviews . org//rr-2004-10 
foliation $M_{t}$ of space-time and reduce the unruly freedom in the choice of gauge conditions that numerical simulations currently face. A second result of interest to numerical relativity is the following. Let a space-time $\left(\mathcal{M}, g_{a b}\right)$ admit a $\mathrm{DH} H$ which asymptotes to the event horizon. Let $M_{0}$ be any partial Cauchy surface in $\left(\mathcal{M}, g_{a b}\right)$ which intersects $H$ in one of the marginally trapped surfaces, say $S_{0}$. Then, $S_{0}$ is the outermost marginally trapped surface - i.e., apparent horizon in the numerical relativity terminology - on $M_{0}$. 


\section{Area Increase Law}

As mentioned in the introduction, the dynamical horizon framework has led to a monotonicity formula governing the growth of black holes. In this section, we summarize this result. Our discussion is divided into three parts. The first spells out the strategy, the second presents a brief derivation of the basic formula, and the third is devoted to interpretational issues.

\subsection{Preliminaries}

The first law of black hole mechanics (1) tells us how the area of the black hole increases when it makes a transition from an initial equilibrium state to a nearby equilibrium state. The question we want to address is: Can one obtain an integral generalization to incorporate fully dynamical situations? Attractive as this possibility seems, one immediately encounters a serious conceptual and technical problem. For, the generalization requires, in particular, a precise notion of the flux of gravitational energy across the horizon. Already at null infinity, the expression of the gravitational energy flux is subtle: One needs the framework developed by Bondi, Sachs, Newman, Penrose, and others to introduce a viable, gauge invariant expression of this flux $[54,33,185]$. In the strong field regime, there is no satisfactory generalization of this framework and, beyond perturbation theory, no viable, gauge invariant notion of the flux of gravitational energy across a general surface.

Yet, there are at least two general considerations that suggest that something special may happen on DHs. Consider a stellar collapse leading to the formation of a black hole. At the end of the process, one has a black hole and, from general physical considerations, one expects that the energy in the final black hole should equal the total matter plus gravitational energy that fell across the horizon. Thus, at least the total integrated flux across the horizon should be well defined. Indeed, it should equal the depletion of the energy in the asymptotic region, i.e., the difference between the ADM energy and the energy radiated across future null infinity. The second consideration involves the Penrose inequality [157] introduced in Section 1. Heuristically, the inequality leads us to think of the radius of a marginally trapped surface as a measure of the mass in its interior, whence one is led to conclude that the change in the area is due to influx of energy. Since a DH is foliated by marginally trapped surfaces, it is tempting to hope that something special may happen, enabling one to define the flux of energy and angular momentum across it. This hope is borne out.

In the discussion of DHs (Sections 3 and 4.2) we will use the following conventions (see Figure 5). The DH is denoted by $H$ and marginally trapped surfaces that foliate it are referred to as crosssections. The unit, time-like normal to $H$ is denoted by $\widehat{\tau}^{a}$ with $g_{a b} \widehat{\tau}^{a} \widehat{\tau}^{b}=-1$. The intrinsic metric and the extrinsic curvature of $H$ are denoted by $q_{a b}:=g_{a b}+\widehat{\tau}_{a} \widehat{\tau}_{b}$ and $K_{a b}:=q_{a}{ }^{c} q_{b}{ }^{d} \nabla_{c} \widehat{\tau}_{d}$, respectively. $D$ is the derivative operator on $H$ compatible with $q_{a b}, \mathcal{R}_{a b}$ its Ricci tensor, and $\mathcal{R}$ its scalar curvature. The unit space-like vector orthogonal to $S$ and tangent to $H$ is denoted by $\widehat{r}^{a}$. Quantities intrinsic to $S$ are generally written with a tilde. Thus, the two-metric on $S$ is $\widetilde{q}_{a b}$ and the extrinsic curvature of $S \subset H$ is $\widetilde{K}_{a b}:=\widetilde{q}_{a}{ }^{c} \widetilde{q}_{b}{ }^{d} D_{c} \widehat{r}_{d}$; the derivative operator on $\left(S, \widetilde{q}_{a b}\right)$ is $\widetilde{D}$ and its Ricci tensor is $\widetilde{\mathcal{R}}_{a b}$. Finally, we fix the rescaling freedom in the choice of null normals to crosssections via $\ell^{a}:=\widehat{\tau}^{a}+\widehat{r}^{a}$ and $n^{a}:=\widehat{\tau}^{a}-\widehat{r}^{a}$ (so that $\ell^{a} n_{a}=-2$ ). To keep the discussion reasonably focused, we will not consider gauge fields with non-zero charges on the horizon. Inclusion of these fields is not difficult but introduces a number of subtleties and complications which are irrelevant for numerical relativity and astrophysics.

Living Reviews in Relativity

http: //www. livingreviews . org/Irr-2004-10 


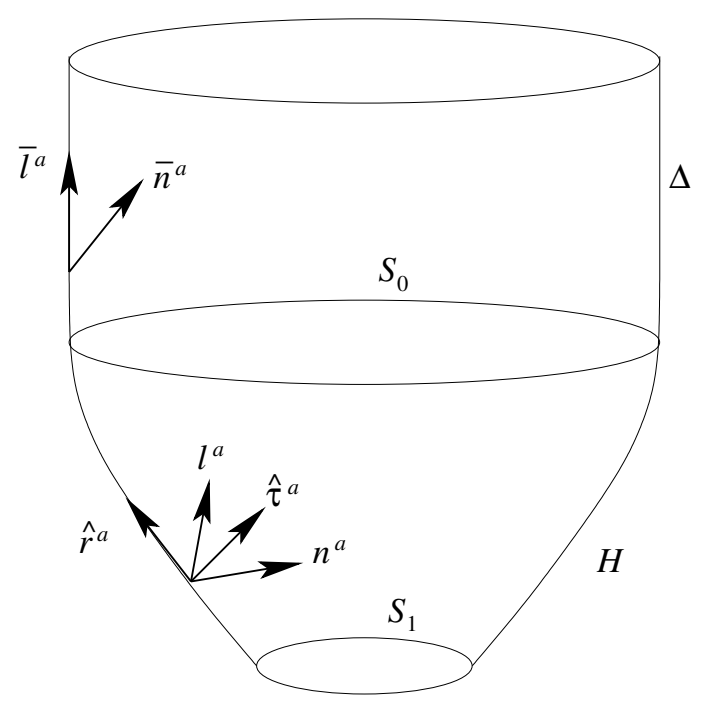

Figure 5: $H$ is a dynamical horizon, foliated by marginally trapped surfaces $S$. $\widehat{\tau}^{a}$ is the unit time-like normal to $H$ and $\widehat{r}^{a}$ the unit space-like normal within $H$ to the foliations. Although $H$ is space-like, motions along $\widehat{r}^{a}$ can be regarded as 'time evolution with respect to observers at infinity'. In this respect, one can think of $H$ as a hyperboloid in Minkowski space and $S$ as the intersection of the hyperboloid with space-like planes. In the figure, $H$ joins on to a weakly isolated horizon $\Delta$ with null normal $\bar{\ell}^{a}$ at a cross-section $S_{0}$.

\subsection{Area increase law}

The qualitative result that the area $a_{S}$ of cross-sections $S$ increases monotonically on $H$ follows immediately from the definition,

$$
\widetilde{K}=\tilde{q}^{a b} D_{a} \widehat{r}_{b}=\frac{1}{2} \tilde{q}^{a b} \nabla_{a}\left(\ell_{b}-n_{b}\right)=-\frac{1}{2} \Theta_{(n)}>0,
$$

since $\Theta_{(\ell)}=0$ and $\Theta_{(n)}<0$. Hence $a_{S}$ increases monotonically in the direction of $\widehat{r}^{a}$. The non-trivial task is to obtain a quantitative formula for the amount of area increase.

To obtain this formula, one simply uses the scalar and vector constraints satisfied by the Cauchy data $\left(q_{a b}, K_{a b}\right)$ on $H$ :

$$
\begin{aligned}
& H_{S}:=\mathcal{R}+K^{2}-K^{a b} K_{a b}=16 \pi G \bar{T}_{a b} \widehat{\tau}^{a} \widehat{\tau}^{b} \\
& H_{V}^{a}:=D_{b}\left(K^{a b}-K q^{a b}\right)=8 \pi G \bar{T}^{b c} \widehat{\tau}_{c} q_{b}^{a},
\end{aligned}
$$

where

$$
\bar{T}_{a b}=T_{a b}-\frac{1}{8 \pi G} \Lambda g_{a b},
$$

and $T_{a b}$ is the matter stress-energy tensor. The strategy is entirely straightforward: One fixes two cross-sections $S_{1}$ and $S_{2}$ of $H$, multiplies $H_{S}$ and $H_{V}^{a}$ with appropriate lapse and shift fields and integrates the result on a portion $\Delta H \subset H$ which is bounded by $S_{1}$ and $S_{2}$. Somewhat surprisingly, if the cosmological constant is non-negative, the resulting area balance law also provides strong constraints on the topology of cross sections $S$.

Specification of lapse $N$ and shift $N^{a}$ is equivalent to the specification of a vector field $\xi^{a}=$ $N \widehat{\tau}^{a}+N^{a}$ with respect to which energy-flux across $H$ is defined. The definition of a DH provides a preferred direction field, that along $\ell^{a}$. Hence it is natural set $\xi^{a}=N \ell^{a} \equiv N \widehat{\tau}^{a}+N \widehat{r}^{a}$. We 
will begin with this choice and defer the possibility of choosing more general vector fields until Section 4.2.

The object of interest now is the flux of energy associated with $\xi^{a}=N \ell^{a}$ across $\Delta H$. We denote the flux of matter energy across $\Delta H$ by $\mathcal{F}_{\text {matter }}^{(\xi)}$ :

$$
\mathcal{F}_{\text {matter }}^{(\xi)}:=\int_{\Delta H} T_{a b} \widehat{\tau}^{a} \xi^{b} d^{3} V
$$

By taking the appropriate combination of Equations (14) and (15) we obtain

$$
\begin{aligned}
\mathcal{F}_{\text {matter }}^{(\xi)} & =\frac{1}{16 \pi G} \int_{\Delta H} N\left(H_{S}+2 \widehat{r}_{a} H_{V}^{a}\right) d^{3} V \\
& =\frac{1}{16 \pi G} \int_{\Delta H} N\left(\mathcal{R}+K^{2}-K^{a b} K_{a b}+2 \widehat{r}_{a} D_{b}\left(K^{a b}-K q^{a b}\right)\right) d^{3} V .
\end{aligned}
$$

Since $H$ is foliated by compact 2-manifolds $S$, one can perform a $2+1$ decomposition of various quantities on $H$. In particular, one first uses the Gauss-Codazzi equation to express $\mathcal{R}$ in terms of $\widetilde{\mathcal{R}}, \widetilde{K}_{a b}$, and a total divergence. Then, one uses the identity

$$
\widetilde{q}^{a b}\left(K_{a b}+\widetilde{K}_{a b}\right)=\Theta_{(\ell)}=0
$$

to simplify the expression. Finally one sets

$$
\sigma_{a b}=\widetilde{q}^{a c} \widetilde{q}^{b d} \nabla_{a} \ell_{b}, \quad \zeta^{a}=\widetilde{q}^{a b} \widehat{r}^{c} \nabla_{c} \ell_{b}
$$

(Note that $\sigma_{a b}$ is just the shear tensor since the expansion of $\ell^{a}$ vanishes.) Then, Equation (18) reduces to

$$
\int_{\Delta H} N \widetilde{\mathcal{R}} d^{3} V=16 \pi G \int_{\Delta H} \bar{T}_{a b} \widehat{\tau}^{a} \xi^{b} d^{3} V+\int_{\Delta H} N\left(|\sigma|^{2}+2|\zeta|^{2}\right) d^{3} V .
$$

To simplify this expression further, we now make a specific choice of the lapse $N$. We denote by $R$ the area-radius function; thus $R$ is constant on each $S$ and satisfies $a_{S}=4 \pi R^{2}$. Since we already know that area increases monotonically, $R$ is a good coordinate on $H$, and using it the 3 -volume $d^{3} V$ on $H$ can be decomposed as $d^{3} V=|\partial R|^{-1} d R d^{2} V$, where $\partial$ denotes the gradient on $H$. Therefore calculations simplify if we choose

$$
N=|\partial R| \equiv N_{R}
$$

We will set $N_{R} \ell^{a}=\xi_{(R)}^{a}$. Then, the integral on the left side of Equation (21) becomes

$$
\int_{\Delta H} N_{R} \widetilde{\mathcal{R}} d^{3} V=\int_{R_{1}}^{R_{2}} d R \oint \widetilde{\mathcal{R}} d^{2} V=\mathcal{I}\left(R_{2}-R_{1}\right),
$$

where $R_{1}$ and $R_{2}$ are the (geometrical) radii of $S_{1}$ and $S_{2}$, and $\mathcal{I}$ is the Gauss-Bonnet topological invariant of the cross-sections $S$. Substituting back in Equation (21) one obtains

$$
\mathcal{I}\left(R_{2}-R_{1}\right)=16 \pi G \int_{\Delta H}\left(T_{a b}-\frac{\Lambda}{8 \pi G} g_{a b}\right) \widehat{\tau}^{a} \xi_{(R)}^{b} d^{3} V+\int_{\Delta H} N_{R}\left(|\sigma|^{2}+2|\zeta|^{2}\right) d^{3} V .
$$

This is the general expression relating the change in area to fluxes across $\Delta H$. Let us consider its ramifications in the three cases, $\Lambda$ being positive, zero, or negative:

- If $\Lambda>0$, the right side is positive definite whence the Gauss-Bonnet invariant $\mathcal{I}$ is positive definite, and the topology of the cross-sections $S$ of the DH is necessarily that of $S^{2}$.

Living Reviews in Relativity

http: //www . livingreviews . org/Irr-2004-10 
- If $\Lambda=0$, then $S$ is either spherical or toroidal. The toroidal case is exceptional: If it occurs, the matter and the gravitational energy flux across $H$ vanishes (see Section 3.3), the metric $\widetilde{q}_{a b}$ is flat, $\mathcal{L}_{n} \Theta_{(\ell)}=0$ (so $H$ can not be a FOTH), and $\mathcal{L}_{\ell} \Theta_{(\ell)}=0$. In view of these highly restrictive conditions, toroidal DHs appear to be unrelated to the toroidal topology of cross-sections of the event horizon discussed by Shapiro, Teukolsky, Winicour, and others $[121,167,139]$. In the generic spherical case, the area balance law (24) becomes

$$
\frac{1}{2 G}\left(R_{2}-R_{1}\right)=\int_{\Delta H} T_{a b} \widehat{\tau}^{a} \xi_{(R)}^{b} d^{3} V+\frac{1}{16 \pi G} \int_{\Delta H} N_{R}\left(|\sigma|^{2}+2|\zeta|^{2}\right) d^{3} V .
$$

- If $\Lambda<0$, there is no control on the sign of the right hand side of Equation (24). Hence, a priori any topology is permissible. Stationary solutions with quite general topologies are known for black holes which are asymptotically locally anti-de Sitter. Event horizons of these solutions are the potential asymptotic states of these DHs in the distant future.

For simplicity, the remainder of our discussion of DHs will be focused on the zero cosmological constant case with 2-sphere topology.

\subsection{Energy flux due to gravitational waves}

Let us interpret the various terms appearing in the area balance law (25).

The left side of this equation provides us with the change in the horizon radius caused by the dynamical process under consideration. Since the expansion $\Theta_{(\ell)}$ vanishes, this is also the change in the Hawking mass as one moves from the cross section $S_{1}$ to $S_{2}$. The first integral on the right side of this equation is the flux $\mathcal{F}_{\text {matter }}^{(R)}$ of matter energy associated with the vector field $\xi_{(R)}^{a}$. The second term is purely geometrical and accompanies the term representing the matter energy flux. Hence it is interpreted as the flux $\mathcal{F}_{\text {grav }}^{(R)}$ of $\xi_{(R)}^{a}$-energy carried by the gravitational radiation:

$$
\mathcal{F}_{\text {grav }}^{(R)}:=\frac{1}{16 \pi G} \int_{\Delta H} N_{R}\left(|\sigma|^{2}+2|\zeta|^{2}\right) d^{3} V .
$$

A priori, it is surprising that there should exist a meaningful expression for the gravitational energy flux in the strong field regime where gravitational waves can no longer be envisaged as ripples on a flat space-time. Therefore, it is important to subject this interpretation to viability criteria analogous to the 'standard' tests one uses to demonstrate the viability of the Bondi flux formula at null infinity. It is known that it passes most of these tests. However, to our knowledge, the status is still partially open on one of these criteria. The situation can be summarized as follows:

\section{Gauge invariance}

Since one did not have to introduce any structure, such as coordinates or tetrads, which is auxiliary to the problem, the expression is obviously gauge invariant. This is to be contrasted with definitions involving pseudo-tensors or background fields.

\section{Positivity}

The energy flux (26) is manifestly non-negative. In the case of the Bondi flux, positivity played a key role in the early development of the gravitational radiation theory. It was perhaps the most convincing evidence that gravitational waves are not coordinate artifacts but carry physical energy. It is quite surprising that a simple, manifestly non-negative expression can exist in the strong field regime of DHs. One can of course apply our general strategy to any space-like 3 -surface $\bar{H}$, foliated by 2 -spheres. However, if $\bar{H}$ is not a $\mathrm{DH}$, the sign of 
the geometric terms in the integral over $\Delta \bar{H}$ can not be controlled, not even when $\bar{H}$ lies in the black hole region and is foliated by trapped (rather than marginally trapped) surfaces $\bar{S}$. Thus, the positivity of $\mathcal{F}_{\text {grav }}^{(R)}$ is a rather subtle property, not shared by 3 -surfaces which are foliated by non-trapped surfaces, nor those which are foliated by trapped surfaces; one needs a foliation precisely by marginally trapped surfaces. The property is delicately matched to the definition of DHs [31].

\section{Locality}

All fields used in Equation (26) are defined by the local geometrical structures on crosssections of $H$. This is a non-trivial property, shared also by the Bondi-flux formula. However, it is not shared in other contexts. For example, the proof of the positive energy theorem by Witten [188] provides a positive definite energy density on Cauchy surfaces. But since it is obtained by solving an elliptic equation with appropriate boundary conditions at infinity, this energy density is a highly non-local function of geometry. Locality of $\mathcal{F}_{\text {grav }}^{(R)}$ enables one to associate it with the energy of gravitational waves instantaneously falling across any cross section $S$.

\section{Vanishing in spherical symmetry}

The fourth criterion is that the flux should vanish in presence of spherical symmetry. Suppose $H$ is spherically symmetric. Then one can show that each cross-section of $S$ must be spherically symmetric. Now, since the only spherically symmetric vector field and trace-free, second rank tensor field on a 2 -sphere are the zero fields, $\sigma_{a b}=0$ and $\zeta^{a}=0$.

\section{Balance law}

The Bondi-Sachs energy flux also has the important property that there is a locally defined notion of the Bondi energy $E(C)$ associated with any 2-sphere cross-section $C$ of future null infinity, and the difference $E\left(C_{1}\right)-E\left(C_{2}\right)$ equals the Bondi-Sachs flux through the portion of null infinity bounded by $C_{2}$ and $C_{1}$. Does the expression (26) share this property? The answer is in the affirmative: As noted in the beginning of this section, the integrated flux is precisely the difference between the locally defined Hawking mass associated with the crosssection. In Section 5 we will extend these considerations to include angular momentum.

Taken together, the properties discussed above provide a strong support in favor of the interpretation of Equation (26) as the $\xi_{(R)}$-energy flux carried by gravitational waves into the portion $\Delta H$ of the DH. Nonetheless, it is important to continue to think of new criteria and make sure that Equation (26) passes these tests. For instance, in physically reasonable, stationary, vacuum solutions to Einstein's equations, one would expect that the flux should vanish. However, on DHs the area must increase. Thus, one is led to conjecture that these space-times do not admit DHs. While special cases of this conjecture have been proved, a general proof is still lacking. Situation is similar for non-spherical DHs in spherically symmetric space-times.

We will conclude this section with two remarks:

- The presence of the shear term $|\sigma|^{2}$ in the integrand of the flux formula (26) seems natural from one's expectations based on perturbation theory at the event horizon of the Kerr family $[108,66]$. But the term $|\zeta|^{2}$ is new and can arise only because $H$ is space-like rather than null: On a null surface, the analogous term vanishes identically. To bring out this point, one can consider a more general case and allow the cross-sections $S$ to lie on a horizon which is partially null and partially space-like. Then, using a $2+2$ formulation [117] one can show that flux on the null portion is given entirely by the term $|\sigma|^{2}[28]$. However, on the spacelike portion, the term $|\zeta|^{2}$ does not vanish in general. Indeed, on a DH, it cannot vanish in presence of rotation: The angular momentum is given by the integral of $\zeta_{a} \varphi^{a}$, where $\varphi^{a}$ is the rotational symmetry.

Living Reviews in Relativity

http://www. livingreviews . org//rr-2004-10 
- The flux refers to a specific vector field $\xi_{(R)}^{a}$ and measures the change in the Hawking mass associated with the cross-sections. However, this is not a good measure of the mass in presence of angular momentum (see, e.g., [34] for numerical simulations). Generalization of the balance law to include angular momentum is discussed in Section 4.2. 


\section{Black Hole Mechanics}

As mentioned in the introduction, the discovery of the laws of black hole mechanics has led to fundamental insights in both classical and quantum gravity. In this section we discuss how the standard framework tied to stationary space-times can be extended using WIHs and DHs.

\subsection{Mechanics of weakly isolated horizons}

The isolated horizon framework has not only extended black hole mechanics, but it has also led to a deeper insight into the 'origin' of the laws of black hole mechanics. In this section we will summarize these developments using WIHs. Along the way we shall also obtain formulas for the mass and angular momentum of a WIH. For simplicity, in the main part of the discussion, we will restrict ourselves to type II (i.e., axi-symmetric) WIHs on which all matter fields vanish. Generalizations including various types of matter field can be found in [19, 26, 15, 75, 76, 77].

\subsubsection{The zeroth law}

The zeroth law of thermodynamics says that the temperature of a system in thermodynamic equilibrium is constant. Its counterpart for black hole mechanics says that surface gravity of a weakly isolated horizon is constant. This result is non-trivial because the horizon geometry is not assumed to be spherically symmetric; the result holds even when the horizon itself is highly distorted so long as it is in equilibrium. It is established as follows.

Recall from Section 2.1.3 that the notion of surface gravity is tied to the choice of a null normal $\ell^{a}$ of the isolated horizon: $\kappa_{(\ell)}:=\ell^{a} \omega_{a}$. Now, using Equation (5) in Definition 2 (of WIHs), we obtain:

$$
\mathcal{L}_{\ell} \omega_{a}=0 .
$$

Next, recall from Equation (9) that the curl of $\omega_{a}$ is related to the imaginary part of $\Psi_{2}$ :

$$
d \omega=2\left(\operatorname{Im} \Psi_{2}\right) \epsilon
$$

where $\epsilon$ is the natural area 2-form on $\Delta$ satisfying $\mathcal{L}_{\ell} \epsilon=0$ and $\ell \cdot \epsilon=0$. Hence we conclude $\ell \cdot d \omega=0$ which in turn implies that $\kappa_{(\ell)}$ is constant on the horizon:

$$
0=\mathcal{L}_{\ell} \omega=d(\ell \cdot \omega)=d \kappa_{(\ell)} .
$$

This completes the proof of the zeroth law. As the argument shows, given an NEH, the main condition (5) in the definition of a WIH is equivalent to constancy of surface gravity. Note that no restriction has been imposed on $\Psi_{2}$ which determines the mass and angular momentum multipoles [24]: as emphasized above, the zeroth law holds even if the WIH is highly distorted and rapidly rotating.

If electromagnetic fields are included, one can also show that the electric potential is constant on the horizon [26]. Finally, there is an interesting interplay between the zeroth law the action principle. Let us restrict ourselves to space-times which admit a non expanding horizon as inner boundary. Then the standard Palatini action principle is not well defined because the variation produces a non-vanishing surface term at the horizon. The necessary and sufficient condition for this surface term to vanish is precisely that the gravitational (and the electromagnetic) zeroth laws hold [26]. Consequently, the standard action principle is well-defined if inner boundaries are WIHs.

Living Reviews in Relativity

http://www . livingreviews . org/Irr-2004-10 


\subsubsection{Phase space, symplectic structure, and angular momentum}

In field theories, conserved quantities such as energy and angular momentum can be universally defined via a Hamiltonian framework: they are the numerical values of Hamiltonians generating canonical transformations corresponding to time translation and rotation symmetries. In absence of inner boundaries, it is this procedure that first led to the notion of the ADM energy and angular momentum at spatial infinity [7]. At null infinity, it can also be used to define fluxes of Bondi energy and angular momentum across regions of $\mathcal{I}^{+}$[33], and values of these quantities associated with any cross-section of $\mathcal{I}^{+}[18,185]$.

This procedure can be extended to allow inner boundaries which are WIHs. The first ingredient required for a Hamiltonian framework is, of course, a phase space. The appropriate phase space now consists of fields living in a region of space-time outside the black hole, satisfying suitable boundary conditions at infinity and horizon. Let $\mathcal{M}$ be the region of space-time that we are interested in. The boundary of $\mathcal{M}$ consists of four components: the time-like cylinder $\tau$ at spatial infinity, two space-like surfaces $M_{1}$ and $M_{2}$ which are the future and past boundaries of $\mathcal{M}$, and an inner boundary $\Delta$ which is to be the WIH (see Figure 6). At infinity, all fields are assumed to satisfy the fall-off conditions needed to ensure asymptotic flatness. To ensure that $\Delta$ is a type II horizon, one fixes a rotational vector field $\varphi^{a}$ on $\Delta$ and requires that physical fields on $\mathcal{M}$ are such that the induced geometry on $\Delta$ is that of a type II horizon with $\varphi^{a}$ as the rotational symmetry.

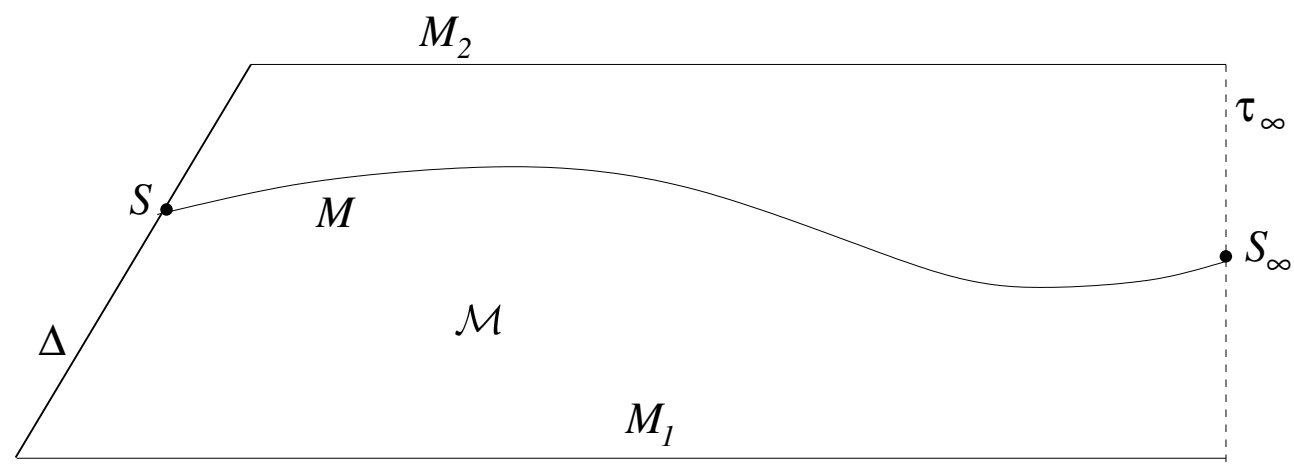

Figure 6: The region of space-time $\mathcal{M}$ under consideration has an internal boundary $\Delta$ and is bounded by two Cauchy surfaces $M_{1}$ and $M_{2}$ and the time-like cylinder $\tau_{\infty}$ at infinity. $M$ is a Cauchy surface in $\mathcal{M}$ whose intersection with $\Delta$ is a spherical cross-section $S$ and the intersection with $\tau_{\infty}$ is $S_{\infty}$, the sphere at infinity.

Two Hamiltonian frameworks are available. The first uses a covariant phase space which consists of the solutions to field equations which satisfy the required boundary conditions [26, 15]. Here the calculations are simplest if one uses a first order formalism for gravity, so that the basic gravitational variables are orthonormal tetrads and Lorentz connections. The second uses a canonical phase space consisting of initial data on a Cauchy slice $M$ of $\mathcal{M}$ [55]. In the gravitational sector, this description is based on the standard ADM variables. Since the conceptual structure underlying the main calculation and the final results are the same, the details of the formalism are not important. For definiteness, in the main discussion, we will use the covariant phase space and indicate the technical modifications needed in the canonical picture at the end.

The phase space $\boldsymbol{\Gamma}$ is naturally endowed with a (pre-)symplectic structure $\boldsymbol{\Omega}$ - a closed 2 -form (whose degenerate directions correspond to infinitesimal gauge motions). Given any two vector fields (i.e., infinitesimal variations) $\delta_{1}$ and $\delta_{2}$ on $\boldsymbol{\Gamma}$, the action $\boldsymbol{\Omega}\left(\delta_{1}, \delta_{2}\right)$ of the symplectic 2 -form on them provides a function on $\boldsymbol{\Gamma}$. A vector field $X$ on $\boldsymbol{\Gamma}$ is said to be a Hamiltonian vector field (i.e., to generate an infinitesimal canonical transformation) if and only if $\mathcal{L}_{X} \boldsymbol{\Omega}=0$. Since the phase 
Abhay Ashtekar and Badri Krishnan

space is topologically trivial, it follows that this condition holds if and only if there is a function $H$ on $\boldsymbol{\Gamma}$ such that $\boldsymbol{\Omega}(\delta, X)=\delta H$ for all vector fields $\delta$. The function $H$ is called a Hamiltonian and $X$ its Hamiltonian vector field; alternatively, $H$ is said to generate the infinitesimal canonical transformation $X$.

Since we are interested in energy and angular momentum, the infinitesimal canonical transformations $X$ will correspond to time translations and rotations. As in any generally covariant theory, when the constraints are satisfied, values of Hamiltonians generating such diffeomorphisms can be expressed purely as surface terms. In the present case, the relevant surfaces are the sphere at infinity and the spherical section $S=M \cap \Delta$ of the horizon. Thus the numerical values of Hamiltonians now consist of two terms: a term at infinity and a term at the horizon. The terms at infinity reproduce the ADM formulas for energy and angular momentum. The terms at the horizon define the energy and angular momentum of the WIH.

Let us begin with angular momentum (see [15] for details). Consider a vector field $\phi^{a}$ on $M$ which satisfies the following boundary conditions: (i) At infinity, $\phi^{a}$ coincides with a fixed rotational symmetry of the fiducial flat metric; and, (ii) on $\Delta$, it coincides with the vector field $\varphi^{a}$. Lie derivatives of physical fields along $\phi^{a}$ define a vector field $X_{(\phi)}$ on $\boldsymbol{\Gamma}$. The question is whether this is an infinitesimal canonical transformation, i.e., a generator of the phase space symmetry. As indicated above, this is the case if and only if there exists a phase space function $J^{(\phi)}$ satisfying:

$$
\delta J^{(\phi)}=\Omega\left(\delta, X_{(\phi)}\right) .
$$

for all variations $\delta$. If such a phase space function $J^{(\phi)}$ exists, it can be interpreted as the Hamiltonian generating rotations.

Now, a direct calculation [16] shows that, in absence of gauge fields on $\Delta$, one has:

$$
\Omega\left(\delta, X_{(\phi)}\right)=-\frac{1}{8 \pi G} \delta \oint_{S}\left[\left(\varphi^{a} \omega_{a}\right)^{2} \epsilon\right]-\delta J_{\mathrm{ADM}}^{(\phi)}=: \delta J^{(\phi)} .
$$

As expected, the expression for $\delta J^{(\phi)}$ consists of two terms: a term at the horizon and a term at infinity. The term at infinity is the variation of the familiar ADM angular momentum $J_{\text {ADM }}^{(\phi)}$ associated with $\phi^{a}$. The surface integral at the horizon is interpreted as the variation of the horizon angular momentum $J_{\Delta}$. Since variations $\delta$ are arbitrary, one can recover, up to additive constants, $J_{\mathrm{ADM}}^{(\phi)}$ and $J_{\Delta}$ from their variations, and these constants can be eliminated by requiring that both of these angular momenta should vanish in static axi-symmetric space-times. One then obtains:

$$
J_{\Delta}:=-\frac{1}{8 \pi} \oint_{S}\left(\omega_{a} \varphi^{a}\right)^{2} \epsilon=-\frac{1}{4 \pi} \oint_{S} f \operatorname{Im}\left[\Psi_{2}\right]^{2} \epsilon
$$

where the function $f$ on $\Delta$ is related to $\varphi^{a}$ by $\partial_{a} f=\epsilon_{b a} \varphi^{b}$. In the last step we have used Equation (28) and performed an integration by parts. Equation (32) is the expression of the horizon angular momentum. Note that all fields that enter this expression are local to the horizon and $\phi^{a}$ is not required to be a Killing field of the space-time metric even in a neighborhood of the horizon. Therefore, $J_{\Delta}$ can be calculated knowing only the horizon geometry of a type II horizon.

We conclude our discussion of angular momentum with some comments:

\section{The Hamiltonian $\boldsymbol{J}^{(\phi)}$}

It follows from Equation (31) and (32) that the total Hamiltonian generating the rotation along $\phi^{a}$ is the difference between the ADM and the horizon angular momenta (apart from a sign which is an artifact of conventions). Thus, it can be interpreted as the angular momentum of physical fields in the space-time region $\mathcal{M}$ outside the black hole.

Living Reviews in Relativity

http: //www. livingreviews . org/Irr-2004-10 


\section{Relation to the Komar integral}

If $\phi^{a}$ happens to be a space-time Killing field in a neighborhood of $\Delta$, then $J_{\Delta}$ agrees with the Komar integral of $\phi^{a}$ [15]. If $\phi^{a}$ is a global, space-time Killing field, then both $J_{\mathrm{ADM}}^{(\phi)}$ as well as $J_{\Delta}$ agree with the Komar integral, whence the total Hamiltonian $J^{(\phi)}$ vanishes identically. Since the fields in the space-time region $\mathcal{M}$ are all axi-symmetric in this case, this is just what one would expect from the definition of $J^{(\phi)}$.

\section{$J_{\Delta}$ for general axial fields $\phi^{a}$}

If the vector field $\phi^{a}$ is tangential to cross-sections of $\Delta, J^{(\phi)}$ continues to the generator of the canonical transformation corresponding to rotations along $\phi^{a}$, even if its restriction $\tilde{\varphi}^{a}$ to $\Delta$ does not agree with the axial symmetry $\varphi^{a}$ of horizon geometries of our phase space fields. However, there is an infinity of such vector fields $\tilde{\varphi}^{a}$ and there is no physical reason to identify the surface term $J_{\Delta}$ arising from any one of them with the horizon angular momentum.

\section{Inclusion of gauge fields}

If non-trivial gauge fields are present at the horizon, Equation (32) is incomplete. The horizon angular momentum $J_{\Delta}$ is still an integral over $S$; however it now contains an additional term involving the Maxwell field. Thus $J_{\Delta}$ contains not only the 'bare' angular momentum but also a contribution from its electromagnetic hair (see [15] for details).

\section{Canonical phase space}

The conceptual part of the above discussion does not change if one uses the canonical phase space [55] in place of the covariant. However, now the generator of the canonical transformation corresponding to rotations has a volume term in addition to the two surface terms discussed above. However, on the constraint surface the volume term vanishes and the numerical value of the Hamiltonian reduces to the two surface terms discussed above.

\subsubsection{Energy, mass, and the first law}

To obtain an expression of the horizon energy, one has to find the Hamiltonian on $\boldsymbol{\Gamma}$ generating diffeomorphisms along a time translation symmetry $t^{a}$ on $\mathcal{M}$. To qualify as a symmetry, at infinity $t^{a}$ must approach a fixed time translation of the fiducial flat metric. At the horizon, $t^{a}$ must be an infinitesimal symmetry of the type II horizon geometry. Thus, the restriction of $t^{a}$ to $\Delta$ should be a linear combination of a null normal $\ell^{a}$ and the axial symmetry vector $\varphi^{a}$,

$$
t^{a}=B_{(\ell, t)} \ell^{a}-\Omega_{(t)} \varphi^{a},
$$

where $B_{(\ell, t)}$ and the angular velocity $\Omega_{(t)}$ are constants on $\Delta$.

However there is subtlety: Unlike in the angular momentum calculation where $\phi^{a}$ is required to approach a fixed rotational vector $\varphi^{a}$ on $\Delta$, the restriction of $t^{a}$ to $\Delta$ can not be a fixed vector field. For physical reasons, the constants $B_{(\ell, t)}$ and $\Omega_{(t)}$ should be allowed to vary from one space-time to another; they are to be functions on phase space. For instance, physically one expects $\Omega_{(t)}$ to vanish on the Schwarzschild horizon but not on a generic Kerr horizon. In the terminology of numerical relativity, unlike $\varphi^{a}$, the time translation $t^{a}$ must be a live vector field. As we shall see shortly, this generality is essential also for mathematical reasons: without it, evolution along $t^{a}$ will not be Hamiltonian!

At first sight, it may seem surprising that there exist choices of evolution vector fields $t^{a}$ for which no Hamiltonian exists. But in fact this phenomenon can also happen in the derivations of the ADM energy for asymptotically flat space-times in the absence of any black holes. Standard treatments usually consider only those $t^{a}$ that asymptote to the same unit time translation at infinity for all space-times included in the phase space. However, if we drop this requirement and 
Abhay Ashtekar and Badri Krishnan

choose a live $t^{a}$ which approaches different asymptotic time-translations for different space-times, then in general there exists no Hamiltonian which generates diffeomorphisms along such a $t^{a}$. Thus, the requirement that the evolution be Hamiltonian restricts permissible $t^{a}$. This restriction can be traced back to the fact that there is a fixed fiducial flat metric at infinity. At the horizon, the situation is the opposite: The geometry is not fixed and this forces one to adapt $t^{a}$ to the space-time under consideration, i.e., to make it live.

Apart from this important caveat, the calculation of the Hamiltonian is very similar to that for angular momentum. First, one evaluates the 1-form $Y_{(t)}$ on $\boldsymbol{\Gamma}$ whose action on any tangent vector field $\delta$ is given by

$$
Y_{(t)}(\delta):=\Omega\left(\delta, X_{(t)}\right),
$$

where $X_{(t)}$ is the vector field on $\boldsymbol{\Gamma}$ induced by diffeomorphisms along $t^{a}$. Once again, $Y_{(t)}(\delta)$ will consist of a surface term at infinity and a surface term at the horizon. A direct calculation yields

$$
Y_{(t)}(\delta)=-\frac{\kappa_{(t)}}{8 \pi G} \delta a_{\Delta}-\Omega_{(t)} \delta J_{\Delta}+\delta E_{\mathrm{ADM}}^{(t)},
$$

where $\kappa_{(t)}:=B_{(\ell, t)} \ell^{a} \omega_{a}$ is the surface gravity associated with the restriction of $t^{a}$ to $\Delta, a_{\Delta}$ is the area of $\Delta$, and $E_{\mathrm{ADM}}^{(t)}$ is the ADM energy associated with $t^{a}$. The first two terms in the right hand side of this equation are associated with the horizon, while the $E_{\mathrm{ADM}}^{t}$ term is associated with an integral at infinity. Since the term at infinity gives the ADM energy, it is natural to hope that terms at the horizon will give the horizon energy. However, at this point, we see an important difference from the angular momentum calculation. Recall that the right hand side of Equation (31) is an exact variation which means that $J^{(\phi)}$ is well defined. However, the right hand side of Equation (35) is not guaranteed to be an exact variation; in other words, $X_{(t)}$ need not be a Hamiltonian vector field in phase space. It is Hamiltonian if and only if there is a phase space function $E_{\Delta}^{(t)}$ - the would be energy of the WIH - satisfying

$$
\delta E_{\Delta}^{(t)}=\frac{\kappa_{(t)}}{8 \pi G} \delta a_{\Delta}+\Omega_{(t)} \delta J_{\Delta}
$$

In particular, this condition implies that, of the infinite number of coordinates in phase space, $E_{\Delta}^{t}$, $\kappa_{(t)}$, and $\Omega_{(t)}$ can depend only on two: $a_{\Delta}$ and $J_{\Delta}$.

Let us analyze Equation (36). Clearly, a necessary condition for existence of $E_{\Delta}^{(t)}$ is just the integrability requirement

$$
\frac{\partial \kappa_{(t)}}{\partial J_{\Delta}}=8 \pi G \frac{\partial \Omega_{(t)}}{\partial a_{\Delta}}
$$

Since $\kappa_{(t)}$ and $\Omega_{(t)}$ are determined by $t^{a}$, Equation (37) is a constraint on the restriction to the horizon of the time evolution vector field $t^{a}$. A vector field $t^{a}$ for which $E_{\Delta}^{(t)}$ exists is called a permissible time evolution vector field. Since Equation (36) is precisely the first law of black hole mechanics, $t^{a}$ is permissible if and only if the first law holds. Thus the first law is the necessary and sufficient condition that the evolution generated by $t^{a}$ is Hamiltonian!

There are infinitely many permissible vector fields $t^{a}$. To construct them, one can start with a suitably regular function $\kappa_{0}$ of $a_{\Delta}$ and $J_{\Delta}$, find $B_{(t)}$ so that $\kappa_{(t)}=\kappa_{0}$, solve Equation (37) to obtain $\Omega_{(t)}$, and find a permissible $t^{a}$ with $t^{a}=B_{(\ell, t)} \ell^{a}-\Omega_{(t)} \varphi^{a}$ on $\Delta$ [15]. Each permissible $t^{a}$ defines a horizon energy $E_{\Delta}^{(t)}$ and provides a first law (36). A question naturally arises: Can one select a preferred $t_{0}^{a}$ or, alternatively, a canonical function $\kappa_{0}\left(a_{\Delta}, J_{\Delta}\right)$ ? Now, thanks to the no-hair theorems, we know that for each choice of $\left(a_{\Delta}, J_{\Delta}\right)$, there is precisely one stationary black hole in vacuum general relativity: the Kerr solution. So, it is natural to set $\kappa_{0}\left(a_{\Delta}, J_{\Delta}\right)=\kappa_{\operatorname{Kerr}}\left(a_{\Delta}, J_{\Delta}\right)$, or, more explicitly,

$$
\kappa_{0}\left(a_{\Delta}, J_{\Delta}\right)=\frac{R_{\Delta}^{4}-4 J_{\Delta}^{2}}{2 R_{\Delta}^{3} \sqrt{R_{\Delta}^{4}+4 J_{\Delta}^{2}}},
$$

Living Reviews in Relativity

http: //www . livingreviews . org/Irr-2004-10 
where $R_{\Delta}$ is the area radius of the horizon, $R_{\Delta}=\left(a_{\Delta} / 4 \pi\right)^{1 / 2}$. Via Equation (37), this choice then leads to

$$
\Omega_{(t)}=\Omega_{\text {Kerr }}\left(a_{\Delta}, J_{\Delta}\right)=\frac{\sqrt{R_{\Delta}^{4}+4 G J_{\Delta}^{2}}}{2 G R_{\Delta}} .
$$

The associated horizon energy is then:

$$
E_{\Delta}^{\left(t_{0}\right)}=\frac{1}{2 G R_{\Delta}} \sqrt{R_{\Delta}^{4}+4 G^{2} J_{\Delta}^{2}} .
$$

This canonical horizon energy is called the horizon mass:

$$
M_{\Delta}:=E_{\Delta}^{\left(t_{0}\right)}
$$

Note that, its dependence on the horizon area and angular momentum is the same as that in the Kerr space-time. Although the final expression is so simple, it is important to keep in mind that this is not just a postulate. Rather, this result is derived using a systematic Hamiltonian framework, following the same overall procedure that leads to the definition of the ADM 4-momentum at spatial infinity. Finally, note that the quantities which enter the first law refer just to physical fields on the horizon; one does not have to go back and forth between the horizon and infinity.

We will conclude with three remarks:

\section{Relation to the ADM and Bondi energy}

Under certain physically reasonable assumptions on the behavior of fields near future timelike infinity $i^{+}$, one can argue that, if the WIH extends all the way to $i^{+}$, then the difference $M_{\mathrm{ADM}}-M_{\Delta}$ equals the energy radiated across future null-infinity [14]. Thus, as one would expect physically, $M_{\Delta}$ is the mass that is left over after all the gravitational radiation has left the system.

\section{Horizon angular momentum and mass}

To obtain a well-defined action principle and Hamiltonian framework, it is essential to work with WIHs. However, the final expressions (32) and (40) of the horizon angular momentum and mass do not refer to the preferred null normals $[\ell]$ used in the transition from an NEH to a WIH. Therefore, the expressions can be used on any NEH. This fact is useful in the analysis of transition to equilibrium (Section 4.3) and numerical relativity (Section 5.1).

\section{Generalizations of the first law}

The derivation of the first law given here can be extended to allow the presence of matter fields at the horizon [26, 15]. If gauge fields are present, the expression of the angular momentum has an extra term and the first law (36) also acquires the familiar extra term ' $\Phi \delta Q$ ', representing work done on the horizon in increasing its charge. Again, all quantities are defined locally on the horizon. The situation is similar in lower [23] and higher [133] space-time dimensions. However, a key difference arises in the definition of the horizon mass. Since the uniqueness theorems for stationary black holes fail to extend beyond the EinsteinMaxwell theory in four space-time dimensions, it is no longer possible to assign a canonical mass to the horizon. However, as we will see in Section 6, the ambiguity in the notion of the horizon mass can in fact be exploited to obtain new insights into the properties of black holes and solitons in these more general theories.

\subsection{Mechanics of dynamical horizons}

The variations $\delta$ in the first law (36) represent infinitesimal changes in equilibrium states of horizon geometries. In the derivation of Section 4.1, these variations relate nearby but distinct space-times 
Abhay Ashtekar and Badri Krishnan

in each of which the horizon is in equilibrium. Therefore Equation (36) is interpreted as the first law in a passive form. Physically, it is perhaps the active form of the first law that is of more direct interest where a physical process, such as the one depicted in the right panel of Figure 1 causes a transition from one equilibrium state to a nearby one. Such a law can be established in the dynamical horizon framework. In fact, one can consider fully non-equilibrium situations, allowing physical processes in a given space-time in which there is a finite - rather than an infinitesimal change in the state of the horizon. This leads to an integral version of the first law.

Our summary of the mechanics of DHs is divided in to three parts. In the first, we begin with some preliminaries on angular momentum. In the second, we extend the area balance law (25) by allowing more general lapse and shift functions, which leads to the integral version of the first law. In the third, we introduce the notion of horizon mass.

\subsubsection{Angular momentum balance}

As one might expect, the angular momentum balance law results from the momentum constraint (15) on the DH $H$. Fix any vector field $\varphi^{a}$ on $H$ which is tangential to all the cross-sections $S$ of $H$, contract both sides of Equation (15) with $\varphi^{a}$, and integrate the resulting equation over the region $\Delta H$ to obtain ${ }^{4}$

$$
\frac{1}{8 \pi G} \oint_{S_{2}} K_{a b} \varphi^{a} \widehat{r}^{b} d^{2} V-\frac{1}{8 \pi G} \oint_{S_{1}} K_{a b} \varphi^{a} \widehat{r}^{b} d^{2} V=\int_{\Delta H}\left(T_{a b} \widehat{\tau}^{a} \varphi^{b}+\frac{1}{16 \pi G}\left(K^{a b}-K q^{a b}\right) \mathcal{L}_{\varphi} q_{a b}\right) d^{3} V .
$$

It is natural to identify the surface integrals with the generalized angular momentum $J_{S}^{\varphi}$ associated with cross-sections $S$ and set

$$
J_{S}^{\varphi}=-\frac{1}{8 \pi G} \oint_{S} K_{a b} \varphi^{a} \widehat{r}^{b} d^{2} V \equiv \frac{1}{8 \pi G} \oint_{S} j^{\varphi} d^{2} V,
$$

where the overall sign ensures compatibility with conventions normally used in the asymptotically flat context, and where we have introduced an angular momentum density $j^{\varphi}:=-K_{a b} \varphi^{a} \widehat{r}^{b}$ for later convenience. The term 'generalized' emphasizes the fact that the vector field $\varphi^{a}$ need not be an axial Killing field even on $S$; it only has to be tangential to our cross-sections. If $\varphi^{a}$ happens to be the restriction of a space-time Killing field to $S$, then $J_{S}^{\varphi}$ agrees with the Komar integral. If the pair $\left(q_{a b}, K_{a b}\right)$ is spherically symmetric on $S$, as one would expect, the angular momenta associated with the rotational Killing fields vanish.

Equation (42) is a balance law; the right side provides expressions of fluxes of the generalized angular momentum across $\Delta H$. The contributions due to matter and gravitational waves are cleanly separated and given by

$$
\mathcal{J}_{\text {matter }}^{\varphi}=-\int_{\Delta H} T_{a b} \widehat{\tau}^{a} \varphi^{b} d^{3} V, \quad \mathcal{J}_{\text {grav }}^{\varphi}=-\frac{1}{16 \pi G} \int_{\Delta H} P^{a b} \mathcal{L}_{\varphi} q_{a b} d^{3} V
$$

with $P^{a b}=K^{a b}-K q^{a b}$, so that

$$
J_{S_{2}}^{\varphi}-J_{S_{1}}^{\varphi}=\mathcal{J}_{\text {matter }}^{\varphi}+\mathcal{J}_{\text {grav }}^{\varphi}
$$

As expected, if $\varphi^{a}$ is a Killing vector of the three-metric $q_{a b}$, then the gravitational angular momentum flux vanishes: $\mathcal{J}_{\text {grav }}^{\varphi}=0$.

As with the area balance law, here we worked directly with the constraint equations rather than with a Hamiltonian framework. However, we could also have used, e.g., the standard ADM

\footnotetext{
${ }^{4}$ Note that we could replace $\bar{T}_{a b}$ with $T_{a b}$ because $g_{a b} \widehat{\tau}^{a} \varphi^{b}=0$. Thus the cosmological constant plays no role in this section.
}

Living Reviews in Relativity

http://www . livingreviews . org//rr-2004-10 
phase space framework based on a Cauchy surface $M$ with internal boundary $S$ and the outer boundary at infinity. If $\phi^{a}$ is a vector field on $M$ which tends to $\varphi^{a}$ on $S$ and to an asymptotic rotational symmetry at infinity, we can ask for the phase space function which generates the canonical transformation corresponding to the rotation generated by $\phi^{a}$. When the constraints are satisfied, as usual the value of this generating function is given by just surface terms. The term at infinity provides the total angular momentum and, as in Section 4.1.2, it is natural to interpret the surface term at $S$ as the $\varphi$-angular momentum of $S$. This term can be expressed in terms of the Cauchy data $\left(\bar{q}_{a b}, \bar{K}_{a b}\right)$ on $M$ as

$$
\bar{J}_{S}^{\varphi}=-\frac{1}{8 \pi G} \oint_{S} \bar{K}_{a b} \varphi^{a} \bar{r}^{b} d^{2} V
$$

where $\bar{r}^{a}$ is the unit normal to $S$ within $M$. However, since the right side involves the extrinsic curvature of $M$, in general the value of the integral is sensitive to the choice of $M$. Hence, the notion of the $\varphi$-angular momentum associated with an arbitrary cross-section is ambiguous. This ambiguity disappears if $\varphi^{a}$ is divergence-free on $S$. In particular, in this case, one has $J_{S}^{\varphi}=\bar{J}_{S}^{\varphi}$. Thus, although the balance law (42) holds for more general vector fields $\varphi^{a}$, it is robust only when $\varphi^{a}$ is divergence-free on $S$. (These considerations shed some light on the interpretation of the field $\zeta^{a}$ in the area balance law (25). For, the form of the right side of Equation (43) implies that the field $\zeta^{a}$ vanishes identically on $S$ if and only if $J_{S}^{\varphi}$ vanishes for every divergence-free $\varphi^{a}$ on $S$. In particular then, if the horizon has non-zero angular momentum, the $\zeta$-contribution to the energy flux can not vanish.)

Finally, for $J_{S}^{\varphi}$ to be interpreted as 'the' angular momentum, $\varphi^{a}$ has to be a symmetry. An obvious possibility is that it be a Killing field of $q_{a b}$ on $S$. A more general scenario is discussed in Section 8.

\subsubsection{Integral form of the first law}

To obtain the area balance law, in Section 3.2 we restricted ourselves to vector fields $\xi_{(R)}^{a}=N_{R} \ell^{a}$, i.e., to lapse functions $N_{R}=|\partial R|$ and shifts $N^{a}=N_{R} \widehat{r}^{a}$. We were then led to a conservation law for the Hawking mass. In the spherically symmetric context, the Hawking mass can be taken to be the physical mass of the horizon. However, as the Kerr space-time already illustrates, in presence of rotation this interpretation is physically incorrect. Therefore, although Equation (25) continues to dictate the dynamics of the Hawking mass even in presence of rotation, a more general procedure is needed to obtain physically interesting conservation laws in this case. In the case of WIHs, the first law incorporating rotations required us to consider suitable linear combinations of $\ell^{a}$ and the rotational symmetry field $\varphi^{a}$ on the horizon. In the same spirit, on DHs, one has to consider more general vector fields than $\xi_{(R)}^{a}$, i.e., more general choices of lapses and shifts.

As on WIHs, one first restricts oneself to situations in which the metric $q_{a b}$ on $H$ admits a Killing field $\varphi^{a}$ so that $J_{S}^{\varphi}$ can be unambiguously interpreted as the angular momentum associated with each $S$. In the case of a WIH, $t^{a}$ was given by $t^{a}=c \ell^{a}+\Omega \varphi^{a}$ and the freedom was in the choice of constants $c$ and $\Omega$. On a DH, one must allow the corresponding coefficients to be 'time-dependent'. The simplest generalization is to choose, in place of $\xi_{(R)}^{a}$, vector fields

$$
t^{a}:=N_{r} \ell^{a}+\Omega \varphi^{a} \equiv N_{r} \widehat{\tau}^{a}+\left(N_{r} \widehat{r}^{a}+\Omega \varphi^{a}\right),
$$

where $\Omega$ is an arbitrary function of $R$, and the lapse $N_{r}$ is given by $N_{r}=|\partial r|$ for any function $r$ of $R$. Note that one is free to rescale $N_{r}$ and $\Omega$ by functions of $R$ so that on each cross-section ('instant of time') one has the same rescaling freedom as on a WIH. One can consider even more general lapse-shift pairs to allow, e.g., for differential rotation (see [31]). 
Using $t^{a}$ in place of $\xi_{(R)}^{a}$, one obtains the following generalization of the area balance equation [31]:

$$
\begin{aligned}
& \frac{r_{2}-r_{1}}{2 G}+\frac{1}{8 \pi G}\left(\oint_{S_{2}} \Omega j^{\varphi} d^{2} V-\oint_{S_{1}} \Omega j^{\varphi} d^{2} V-\int_{\Omega_{1}}^{\Omega_{2}} d \Omega \oint_{S} j^{\varphi} d^{2} V\right)= \\
& \int_{\Delta H} T_{a b} \widehat{\tau}^{a} t^{b} d^{3} V+\frac{1}{16 \pi G} \int_{\Delta H} N_{r}\left(|\sigma|^{2}+2|\zeta|^{2}\right) d^{3} V-\frac{1}{16 \pi G} \int_{\Delta H} \Omega P^{a b} \mathcal{L}_{\varphi} q_{a b} d^{3} V .
\end{aligned}
$$

Note that there is one balance equation for every vector field $t^{a}$ of the form (47); as in Section 4.1, we have an infinite number of relations, now ensured by the constraint part of Einstein's equations.

The right side of Equation (48) can be naturally interpreted as the flux $\mathcal{F}_{\Delta H}^{t}$ of the 'energy' $E^{t}$ associated with the vector field $t^{a}$ across $\Delta H$. Hence, we can rewrite the equation as

$$
\mathcal{F}_{\Delta H}^{t}=\frac{r_{2}-r_{1}}{2 G}+\frac{1}{8 \pi G}\left(\oint_{S_{2}} \Omega j^{\varphi} d^{2} V-\oint_{S_{1}} \Omega j^{\varphi} d^{2} V-\int_{\Omega_{1}}^{\Omega_{2}} d \Omega \oint_{S} j^{\varphi} d^{2} V\right)
$$

If $S_{1}$ and $S_{2}$ are only infinitesimally separated, this integral equation reduces to the differential condition

$$
\begin{aligned}
\delta E_{S}^{t} & =\left.\frac{1}{8 \pi G}\left(\frac{1}{2 R} \frac{d r}{d R}\right)\right|_{S} \delta a_{s}+\Omega \delta J_{S}^{\varphi} \\
& \equiv \frac{\bar{\kappa}}{8 \pi G} \delta a_{S}+\Omega \delta J_{S}^{\varphi} .
\end{aligned}
$$

Thus, the infinitesimal form of Equation (48) is a familiar first law, provided $[(1 / 2 R)(d r / d R)](S)$ is identified as an effective surface gravity on the cross-section $S$. This identification can be motivated as follows. First, on a spherically symmetric DH, it is natural to choose $r=R$. Then the surface gravity reduces to $1 /(2 R)$, just as one would hope from one's experience with the Schwarzschild metric and more generally with static but possibly distorted horizons (See Appendix A of [14]). Under the change $R \mapsto r(R)$, we have $\bar{\kappa}_{r}=(d r / d R) \bar{\kappa}_{R}$, which is the natural generalization of the transformation property $\kappa_{c \ell}=c \kappa_{\ell}$ of surface gravity of WIHs under the change $\ell \mapsto c \ell$. Finally, $\bar{\kappa}_{r}$ can also be regarded as a 2 -sphere average of a geometrically defined surface gravity associated with certain vector fields on $H[56,31]$; hence the adjective 'effective'.

To summarize, Equation (48) represents an integral generalization of the first law of mechanics of weakly isolated horizons to dynamical situations in which the horizon is permitted to make a transition from a given state to one far away, not just nearby. The left side represents the flux $\mathcal{F}_{\Delta H}^{t}$ of the energy associated with the vector field $t^{a}$, analogous to the flux of Bondi energy across a portion of null infinity. A natural question therefore arises: Can one integrate this flux to obtain an energy $E_{S}^{t}$ which depends only on fields defined locally on the cross-section, as is possible at null infinity? As discussed in the next section, the answer is in the affirmative and the procedure leads to a canonical notion of horizon mass.

\subsubsection{Horizon mass}

In general relativity, the notion of energy always refers to a vector field. On DHs, the vector field is $t^{a}$. Therefore, to obtain an unambiguous notion of horizon mass, we need to make a canonical choice of $t^{a}=N_{r} \ell^{a}+\Omega \varphi^{a}$, i.e., of functions $N_{r}$ and $\Omega$ on $H$. As we saw in Section 4.1.3, on WIHs of 4-dimensional Einstein-Maxwell theory, the pair $\left(a_{\Delta}, J_{\Delta}\right)$ suffices to pick a canonical time translation field $t_{0}^{a}$ on $\Delta$. The associated horizon energy $E_{\Delta}^{t_{0}}$ is then interpreted as the mass $M_{\Delta}$. This suggests that the pair $\left(a_{S}, J_{S}^{\varphi}\right)$ be similarly used to make canonical choices $N_{r^{0}}$ and

Living Reviews in Relativity

http://www. livingreviews . org//rr-2004-10 
$\Omega^{0}$ on $S$. Thanks to the black hole uniqueness theorems of the 4-dimensional Einstein-Maxwell theory, this strategy is again viable.

Recall that the horizon surface gravity and the horizon angular velocity in a Kerr solution can be expressed as a function only of the horizon radius $R$ and angular momentum $J$ :

$$
\kappa_{\text {Kerr }}(R, J):=\frac{R^{4}-4 G^{2} J^{2}}{2 R^{3} \sqrt{R^{4}+4 G^{2} J^{2}}}, \quad \Omega_{\mathrm{Kerr}}(R, J):=\frac{2 G J}{R \sqrt{R^{4}+4 G^{2} J^{2}}} .
$$

Given a cross section $S$ of $H$, the idea is to consider the unique Kerr solution in which the horizon area is given by $a_{S}$ and angular momentum by $J_{S}$, and assign to $S$ effective surface gravity $\bar{\kappa}_{S}$ and angular velocity $\Omega_{S}$ through

$$
\bar{\kappa}_{S}:=\kappa_{\mathrm{Kerr}}\left(R_{S}, J_{S}^{\varphi}\right), \quad \Omega_{S}:=\Omega_{\mathrm{Kerr}}\left(R_{S}, J_{S}^{\varphi}\right)
$$

Repeating this procedure on every cross-section, one obtains functions $\bar{\kappa}^{0}(R)$ and $\Omega^{0}(R)$ on $H$, since $J^{\varphi}$ is a function of $R$ alone. The definition of the effective surface gravity then determines a function $r^{0}$ of $R$ and hence $N_{r^{0}}$ uniquely. Thus, using Equation (51), one can select a canonical vector field $t_{0}^{a}$ and Equation (49) then provides a canonical balance law:

$$
\mathcal{F}_{\Delta H}^{t_{0}}=\frac{r_{2}^{0}-r_{1}^{0}}{2 G}+\frac{1}{8 \pi G}\left(\oint_{S_{2}} \Omega^{0} j^{\varphi} d^{2} V-\oint_{S_{1}} \Omega^{0} j^{\varphi} d^{2} V-\int_{\Omega_{1}^{0}}^{\Omega_{2}^{0}} d \Omega^{0} \oint_{S} j^{\varphi} d^{2} V\right) .
$$

The key question is whether this equation is integrable, i.e., if

$$
\mathcal{F}_{\Delta H}^{t_{0}}=E_{S_{2}}^{t_{0}}-E_{S_{1}}^{t_{0}}
$$

for some $E_{S}^{t_{0}}$ which depends locally on fields defined on $S$. The answer is in the affirmative. Furthermore, the expression of $E^{t_{0}}$ is remarkably simple and is identified with the horizon mass:

$$
M(R):=E^{t_{0}}(R)=\frac{\sqrt{R^{4}+4 G^{2} J^{2}}}{2 G R} .
$$

Thus, on any cross-section $S, M_{S}$ is just the mass of the Kerr space-time which has horizon area $a_{S}$ and angular momentum $J_{S}^{\varphi}$ : As far as the mass is concerned, one can regard the DH as an evolution through 'a sequence of Kerr horizons'. The non-triviality of the result lies in the fact that, although this definition of mass is so 'elementary', thanks to the balance law (48) it obeys a Bondi-type flux formula,

$$
M_{S_{2}}-M_{S_{1}}=\int_{\Delta H} T_{a b} \widehat{\tau}^{a} t_{0}^{b} d^{3} V+\frac{1}{16 \pi G} \int_{\Delta H} N_{r^{0}}\left(|\sigma|^{2}+2|\zeta|^{2}\right) d^{3} V
$$

for a specific vector field $t_{0}^{a}=N_{r^{0}} \ell^{a}+\Omega^{0} \varphi^{a}$, where each term on the right has a well-defined physical meaning. Thus, DHs admit a locally-defined notion of mass and an associated, canonical conservation law (56). The availability of a mass formula also provides a canonical integral version of the first law through Equation (48):

$$
M_{S_{2}}-M_{S_{1}}=\frac{r_{2}^{0}-r_{1}^{0}}{2 G}+\frac{1}{8 \pi G}\left(\oint_{S_{2}} \Omega^{0} j^{\varphi} d^{2} V-\oint_{S_{1}} \Omega^{0} j^{\varphi} d^{2} V-\int_{\Omega_{1}^{0}}^{\Omega_{2}^{0}} d \Omega^{0} \oint_{S} j^{\varphi} d^{2} V\right) .
$$

The infinitesimal version of this equation yields the familiar first law $\delta M=(\bar{\kappa} / 8 \pi G) \delta a+\Omega \delta J$.

On weakly isolated as well as dynamical horizons, area $a$ and angular momentum $J$ arise as the fundamental quantities and mass is expressed in terms of them. The fact that the horizon mass is the same function of $a$ and $J$ in both dynamical and equilibrium situations is extremely convenient for applications to numerical relativity [34]. Conceptually, this simplicity is a direct consequence of the first law and the non-triviality lies in the existence of a balance equation (48), which makes it possible to integrate the first law. 


\subsection{Passage of dynamical horizons to equilibrium}

In physical situations, such as a gravitational collapse or black hole mergers, one expects the dynamical horizon to approach equilibrium at late times and become isolated. Because of back scattering, generically the approach is only asymptotic. However, the back scattering is generally quite weak and in simulations, within numerical errors, equilibrium is reached at finite times. The passage to equilibrium can be studied in detail in Vaidya solutions discussed in Section 2.2.2. Moreover, in spherically symmetric examples such as these solutions, exact equilibrium can be reached at a finite time (see right panel of Figure 4). The question then arises: In these situations, do various notions introduced on dynamical horizons go over smoothly to those introduced on WIHs? This issue has been analyzed only in a preliminary fashion [56, 31]. In this section we will summarize the known results.

First, if the dynamical horizon is a FOTH, as the flux of matter and shear across $H$ tends to zero, $H$ becomes null and furthermore a non-expanding horizon. By a suitable choice of null normals, it can be made weakly isolated. Conditions under which it would also become an isolated horizon are not well-understood. Fortunately, however, the final expressions of angular momentum and horizon mass refer only to that structure which is already available on non-expanding horizons (although, as we saw in Section 4.1, the underlying Hamiltonian framework does require the horizon to be weakly isolated $[26,15])$. Therefore, it is meaningful to ask if the angular momentum and mass defined on the DHs match with those defined on the non-expanding horizons. In the case when the approach to equilibrium is only asymptotic, it is rather straightforward to show that the answer is in the affirmative.

In the case when the transition occurs at a finite time, the situation is somewhat subtle. First, we now have to deal with both regimes and the structures available in the two regimes are entirely different. Second, since the intrinsic metric becomes degenerate in the transition from the dynamical to isolated regimes, limits are rather delicate. In particular, the null vector field $\ell^{a}=\widehat{\tau}^{a}+\widehat{r}^{a}$ on $H$ diverges, while $n^{a}=\widehat{\tau}^{a}-\widehat{r}^{a}$ tends to zero at the boundary. A priori therefore, it is not at all clear that angular momentum and mass would join smoothly if the transition occurs at a finite time. However, a detailed analysis shows that the two sets of notions in fact agree.

More precisely, one has the following results. Let $\mathcal{Q}=H \cup \Delta$ be a $C^{k+1} 3$-manifold (with $k \geq 2$ ), topologically $\mathbb{S}^{2} \times \mathbb{R}$ as in the second Penrose diagram of Figure 4. Let the space-time metric $g_{a b}$ in a neighborhood of $\mathcal{Q}$ be $C^{k}$. The part $H$ of $\mathcal{Q}$ is assumed to have the structure of a $\mathrm{DH}$ and the part $\Delta$ of a non-expanding horizon. Finally, the pull-back $q_{a b}$ of $g_{a b}$ to $\mathcal{Q}$ is assumed to admit an axial Killing field $\varphi^{a}$. Then we have:

- The angular momentum $J^{\varphi}$ and the mass $M$ defined in the two regimes agree on the boundary between $H$ and $\Delta$.

- The vector field $t_{0}^{a}$ defined on $H$ and used in the definition of mass matches with a preferred vector field $t_{0}^{a}$ used to define mass on $\Delta$.

This agreement provides an independent support in favor of the strategy used to introduce the notion of mass in the two regimes.

Living Reviews in Relativity

http://www. livingreviews .org/lrr-2004-10 


\section{Applications in Numerical Relativity}

By their very nature, numerical simulations of space-times are invariably tied to choices of coordinates, gauge conditions, dynamical variables, etc. Therefore, it is non-trivial to extract from them gauge invariant physics, especially in the strong curvature regions. Traditionally, the analytical infrastructure available for this purpose has been based on properties of the Kerr solution and its perturbations. However, a priori it is not clear if this intuition is reliable in the fully dynamical, strong curvature regime. On the numerical side, a number of significant advances have occurred in this area over the past few years. In particular, efficient algorithms have been introduced to find apparent horizons (see, e.g., $[6,168,177])$, black hole excision techniques have been successfully implemented [73, 3], and the stability of numerical codes has steadily improved [64]. To take full advantage of these ongoing improvements, one must correspondingly 'upgrade' the analytical infra-structure so that one can extract physics more reliably and with greater accuracy.

These considerations provided stimulus for a significant body of research at the interface of numerical relativity and the dynamical and isolated horizon frameworks. In this section, we will review the most important of these developments. Section 5.1 summarizes calculations of mass and angular momentum of black holes. Section 5.2 discusses applications to problems involving initial data. Specifically, we discuss the issue of constructing the 'quasi-equilibrium initial data' and the calculation of the gravitational binding energy for a binary black hole problem. Section 5.3 describes how one can calculate the source multipole moments for black holes, and Section 5.4 presents a 'practical' approach for extracting gauge invariant waveforms. Throughout this section we assume that vacuum equations hold near horizons.

\subsection{Numerical computation of black hole mass and angular momentum}

As we saw in Section 4, the mechanics of IHs and DHs provides expressions of angular momentum and mass of the horizon. These expressions involve geometric quantities defined intrinsically on the IH $\Delta$ and DH $H$. Numerical simulations, on the other hand, deal with the 3-metric $\bar{q}_{a b}$ and extrinsic curvature $\bar{K}_{a b}$ on (partial) Cauchy surfaces $M$. Therefore, the first task is to recast the formulas in terms of this Cauchy data.

Simulations provide us with a foliation of space-time $\mathcal{M}$ by partial Cauchy surfaces $M$, each of which has a marginally trapped 2-surface $S$ as (a connected component of) its inner boundary. The world tube of these 2-surfaces is a candidate for a $\mathrm{DH}$ or an IH. If it is space-like, it is a $\mathrm{DH}$ $H$ and if it is null (or, equivalently, if the shear $\sigma_{a b}$ of the outward null normal to $S$ is zero) it is a WIH $\Delta$. The situation is depicted in Figure 7 . It is rather simple to numerically verify if these restrictions are met. To calculate mass and angular momentum, one assumes that the intrinsic 2-metric on the cross-sections $S$ admits a rotational Killing field $\varphi^{a}$ (see, however, Section 8 for weakening of this assumption). A rather general and convenient method, based on the notion of Killing transport, has been introduced and numerically implemented to explicitly find this vector field $\varphi^{a}[85]$.

Let us first suppose that, in a neighborhood of the cross-section $S$ of interest, the world tube of marginally trapped surfaces constitutes an IH. Then the task is to recast Equation (32) in terms of the Cauchy data $\left(\bar{q}_{a b}, \bar{K}_{a b}\right)$ on $M$. This task is also straightforward [85] and one arrives at ${ }^{5}$ :

$$
J_{\Delta}=-\frac{1}{8 \pi G} \oint_{S} \varphi^{a} R^{b} \bar{K}_{a b} d^{2} V,
$$

where $R^{a}$ is the unit radial normal to $S$ in $M$. This formula is particularly convenient numerically since it involves the integral of a single component of the extrinsic curvature.

\footnotetext{
${ }^{5}$ This formula has a different sign from that given in [85] due to a difference in the sign convention in the definition of the extrinsic curvature.
} 


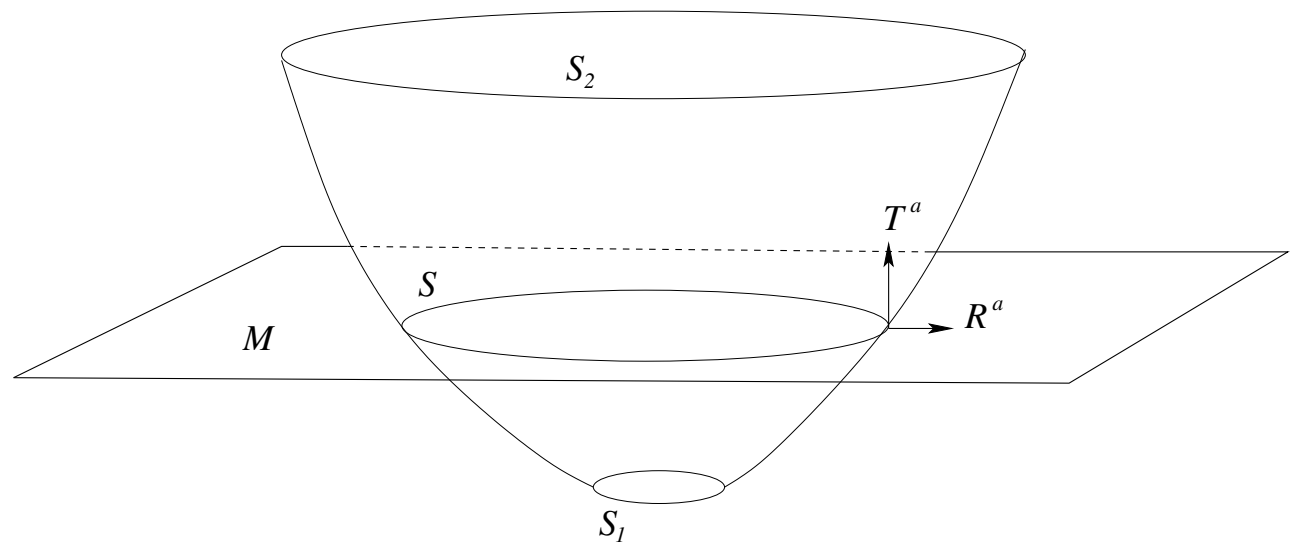

Figure 7: The world tube of apparent horizons and a Cauchy surface $M$ intersect in a 2-sphere $S$. $T^{a}$ is the unit time-like normal to $M$ and $R^{a}$ is the unit space-like normal to $S$ within $M$.

Now consider the dynamical regime, i.e., assume that, in a small neighborhood of $S$, the world tube of marginally trapped surfaces is a DH $H$. The angular momentum formula (46) on DHs involves the Cauchy data on $H$. However, it is easy to show [31] that it equals the expression (58) involving Cauchy data on $M$. Thus, Equation (58) is in fact applicable in both the isolated and dynamical regimes. The availability of a single formula is extremely convenient in numerical simulations. From now on, we will drop the subscript $\Delta$ and denote the angular momentum of $S$ simply by $J_{S}^{(\varphi)}$ :

$$
J_{S}^{(\varphi)}=-\frac{1}{8 \pi G} \oint_{S} \varphi^{a} R^{b} \bar{K}_{a b} d^{2} V
$$

$J_{S}^{(\varphi)}$ is the intrinsic angular momentum (i.e., spin) of the black hole at the instant of 'time' represented by $S$. Note that Equation (59) differs from the standard formula for the ADM angular momentum $J_{\mathrm{ADM}}$ only in that the integral is over the apparent horizon instead of the sphere at infinity. Finally, we emphasize that one only assumes that $\varphi^{a}$ is a rotational Killing field of the intrinsic 2-metric on $S$; it does not have to extend to a Killing field even to a neighborhood of $S$. In fact, the expressions (32) and (46) on IHs and DHs are meaningful if $\varphi^{a}$ is replaced by any vector field $\tilde{\phi}^{a}$ tangential to $S$; on $\Delta$ the expression is conserved and on $H$ it satisfies a balance law. Furthermore, they both equal Equation (59) if $\tilde{\varphi}^{a}$ is divergence-free; it has to Lie-drag only the area 2 -form (rather than the intrinsic metric) on $S$. However, since $S$ admits an infinity of divergencefree vector fields $\tilde{\varphi}^{a}$, there is no a priori reason to interpret $J_{S}^{(\tilde{\phi})}$ as the 'angular momentum' of the black hole. For this interpretation to be meaningful, $\tilde{\varphi}^{a}$ must be chosen 'canonically'. The most unambiguous way to achieve this is to require that it be a Killing field of the intrinsic geometry of $S$. However, in Section 8 we will introduce a candidate vector field which could be used in more general situations which are only 'near axi-symmetry'.

Having calculated $J_{S}$, it is easy to evaluate the mass $M_{S}$ using Equation (40):

$$
M_{S}=\frac{1}{2 G R_{\Delta}} \sqrt{R_{\Delta}^{4}+4 G^{2} J_{\Delta}^{2}}
$$

This method for calculating $J_{S}$ and $M_{S}$ has already been used in numerical simulations, particularly in the analysis of the gravitational collapse of a neutron star to a black hole [34, 48]. It is found that this method is numerically more accurate than the other commonly used methods.

Living Reviews in Relativity

http: //www. livingreviews. org/Irr-2004-10 


\section{Comparison with other methods}

The approach summarized above has the key advantage that it is rooted in Hamiltonian methods which can be universally used to define conserved quantities. In particular, it is a natural extension of the approach used to arrive at the ADM and Bondi-Sachs quantities at spatial and null infinity [7, 18, 185]. From more 'practical' considerations, it has three important features:

- The procedure does not presuppose that the horizon geometry is precisely that of the Kerr horizon.

- It is coordinate independent.

- It only requires data that is intrinsic to the apparent horizon.

None of the commonly used alternatives share all three of these features.

Before the availability of the $\mathrm{IH}$ and $\mathrm{DH}$ frameworks, standard procedures of calculating angular momentum were based on properties of the Kerr geometry. The motivation comes from the common belief, based on black-hole uniqueness theorems, that a black hole created in a violent event radiates away all its higher multipole moments and as it settles down, its near horizon geometry can be approximated by that of the Kerr solution. The strategy then is to identify the geometry of $S$ with that of a suitable member of the Kerr family and read off the corresponding angular momentum and mass parameters.

The very considerations that lead one to this strategy also show that it is not suitable in the dynamical regime where the horizon may be distorted and not well-approximated by any Kerr horizon. For horizons which have very nearly reached equilibrium, the strategy is physically well motivated. However, even in this case, one has to find a way to match the horizon of the numerical simulation with that of a specific member in the Kerr family. This is non-trivial because the coordinate system used in the given simulation will, generically, not bear any relation to any of the standard coordinate systems used to describe the Kerr solution. Thus, one cannot just look at, say, a metric component to extract mass and angular momentum.

A semi-heuristic but most-commonly used procedure is the great circle method. It is based on an observation of the properties of the Kerr horizon made by Smarr [169] using Kerr-Schild coordinates. Let $L_{\mathrm{e}}$ be the length of the equator and $L_{\mathrm{p}}$ the length of a polar meridian on the Kerr horizon, where the equator is the coordinate great circle of maximum proper length and a polar meridian is a great circle of minimum proper length. Define a distortion parameter $\delta$ as $\delta=\left(L_{\mathrm{e}}-L_{\mathrm{p}}\right) / L_{\mathrm{e}}$. The knowledge of $\delta$, together with one other quantity such as the area, $L_{\mathrm{e}}$, or $L_{\mathrm{p}}$, is sufficient to find the parameters $m$ and $a$ of the Kerr geometry. However, difficulties arise when one wishes to use these ideas to calculate $M$ and $a$ for a general apparent horizon $S$. For, notions such as great circles, equator or polar meridian are all highly coordinate dependent. Indeed, if we represent the standard two-metric on the Kerr horizon in different coordinates, the great circles in one coordinate system will not agree with great circles in the other system. Therefore, already for the Kerr horizon, two coordinate systems will lead to different answers for $M$ and $a$ ! In certain specific situations where one has a good intuition about the coordinate system being used and the physical situation being modelled, this method can be useful as a quick way of estimating angular momentum. However, it has the conceptual drawback that it is not derived from a well-founded, general principle and the practical drawback that it suffers from too many ambiguities. Therefore it is inadequate as a general method.

Problems associated with coordinate dependence can be satisfactorily resolved on axi-symmetric horizons, even when the coordinate system used in the numerical code is not adapted 
to the axial symmetry. The idea is to use the orbits of the Killing vector as analogs of the lines of latitude on a metric two-sphere. The analog of the equator is then the orbit of the Killing vector which has maximum proper length. This defines $L_{\mathrm{e}}$ in an invariant way. The north and south poles are the points where the Killing vector vanishes, and the analog of $L_{\mathrm{p}}$ is the length of a geodesic joining these two points. (Because of axial symmetry, all geodesics joining the poles will have the same length). This geodesic is necessarily perpendicular to the Killing vector. Hence one just needs to find the length of a curve joining the north and south poles which is everywhere perpendicular to the Killing orbits. With $L_{\mathrm{e}}$ and $L_{\mathrm{p}}$ defined in this coordinate invariant way, one can follow the same procedure as in the great circle method to calculate the mass and angular momentum. This procedure has been named [85] the generalized great circle method.

How does the generalized great circle method compare to that based on IHs and DHs? Since one must find the Killing vector, the first step is the same in the two cases. In the IH and DH method, one is then left simply with an integration of a component of the extrinsic curvature on the horizon. In the generalized great circle method, by contrast, one has to determine the orbit of the Killing vector with maximum length and also to calculate the length of a curve joining the poles which is everywhere orthogonal to the Killing orbits. Numerically, this requires more work and the numerical errors are at least as large as those in the IHDH method. Thus, even if one ignores conceptual considerations involving the fundamental meaning of conserved quantities, and furthermore restricts oneself to the non-dynamical regime, the practical simplicity of the great circle method is lost when it is made coordinate invariant. To summarize, conceptually, Equations (59) and (60) provide the fundamental definitions of angular momentum and mass, while the great circle method provides a quick way of estimating these quantities in suitable situations. By comparing with Equations (59) and (60), one can calculate errors and sharpen intuition on the reliability of the great circle method.

A completely different approach to finding the mass and angular momentum of a black hole in a numerical solution is to use the concept of a Killing horizon. Assume the existence of Killing vectors in the neighborhood of the horizon so that mass and angular momentum are defined as the appropriate Komar integrals. This method is coordinate independent and does not assume, at least for angular momentum, that the near horizon geometry is isometric with the Kerr geometry. But it has two disadvantages. First, since the Komar integral can involve derivatives of the Killing field away from the horizon, one has to find the Killing fields in a neighborhood of the horizon. Second, existence of such a stationary Killing vector is a strong assumption; it will not be satisfied in the dynamical regime. Even when the Killing field exists, computationally it is much more expensive to find it in a neighborhood of the horizon rather than the horizon itself. Finally, it is not a priori clear how the stationary Killing vector is to be normalized if it is only known in a neighborhood of the horizon. In a precise sense, the isolated horizon framework extracts just the minimum amount of information from a Killing horizon in order to carry out the Hamiltonian analysis and define conserved quantities by by-passing these obstacles.

\section{$5.2 \quad$ Initial data}

In order to accurately calculate the gravitational waveforms for, say, the coalescence of binary black holes, one must begin with astrophysically relevant initial data. While there has been some progress on the construction of such data, the general problem is yet to be solved (see, e.g., [70] for a recent review). However, there has been notable progress in the case of black holes which are in quasi-equilibrium. Physically, this situation arises when the black holes are sufficiently far apart for their orbits to be quasi-periodic. In his section, we will first summarize this development [72, 124]

Living Reviews in Relativity

http://www . livingreviews . org//rr-2004-10 
and then review an approach to the calculation of the binding energy in the initial data. The discussion is based on the IH framework.

\subsubsection{Boundary conditions at the inner boundary}

Consider the problem of constructing initial data $\left(\bar{q}_{a b}, \bar{K}_{a b}\right)$ on $M$, representing a binary black hole system. This problem has two distinct aspects. The first has to do with the inner boundaries. A natural avenue to handle the singularities is to 'excise' the region contained in each black hole (see, e.g., $[3,73])$. However, this procedure creates inner boundaries on $M$ and one must specify suitable boundary conditions there. The boundary conditions should be appropriate for the elliptic system of constraint equations, and they should also capture the idea that the excised region represents black holes which have certain physical parameters and which are in quasi-equilibrium at the 'instant' represented by $M$. The second aspect of the problem is the choice of the free data in the bulk. To be of physical interest, not only must the free data satisfy the appropriate boundary conditions, but the values in the bulk must also have certain properties. For example, we might want the black holes to move in approximately circular orbits around each other, and require that there be only a minimal amount of spurious gravitational radiation in the initial data. The black holes would then remain approximately isolated for a sufficiently long time, and orbit around each other before finally coalescing.

While a fully satisfactory method of prescribing such initial data is still lacking, there has been significant progress in recent years. When the black holes are far apart and moving on approximately circular orbits, one might expect the trajectory of the black holes to lie along an approximate helical Killing vector [53, 91, 103, 104, 4]. Using concepts from the helical Killing vector approximation and working in the conformal thin-sandwich decomposition of the initial data [192], Cook has introduced the 'quasi-equilibrium' boundary conditions which require that each of the black holes be in instantaneous equilibrium [72]; see also [190, 74] for a similar approach. The relation between these quasi-equilibrium boundary conditions and the isolated horizon formalism has also been recently studied [124].

In this section, we consider only the first aspect mentioned above, namely the inner boundaries. Physically, the quasi-equilibrium approximation ought to be valid for time intervals much smaller than other dynamical time scales in the problem, and the framework assumes only that the approximation holds infinitesimally 'off $M$ '. So, in this section, the type II NEH $\Delta$ will be an infinitesimal world tube of apparent horizons. We assume that there is an axial symmetry vector $\varphi^{a}$ on the horizon, although, as discussed in Section 8, this assumption can be weakened.

Depending on the degree of isolation one wants to impose on the individual black holes, the inner boundary may be taken to be the cross-section of either an NEH, WIH, or an IH. The strategy is to first start with an NEH and impose successively stronger conditions if necessary. Using the local geometry of intersections $S$ of $M$ with $\Delta$, one can easily calculate the area radius $R_{\Delta}$, the angular momentum $J_{\Delta}$ given by Equation (59), the canonical surface gravity $\kappa=\kappa_{\mathrm{Kerr}}\left(R_{\Delta}, J_{\Delta}\right)$ given by Equation (38), and angular velocity $\Omega=\Omega_{\mathrm{Kerr}}\left(R_{\Delta}, J_{\Delta}\right)$ given by Equation (39). (Note that while a WIH structure is used to arrive at the expressions for $J_{\Delta}, \kappa$, and $\Omega$, the expressions themselves are unambiguously defined also on a NEH.) These considerations translate directly into restrictions on the shift vector $\beta^{a}$ at the horizon. If, as in Section 4.1.3, one requires that the restriction of the evolution vector field $t^{a}$ to $\Delta$ be of the form

$$
t^{a}=B \ell_{0}^{a}-\Omega_{\mathrm{Kerr}} \varphi^{a}=B T^{a}+\left(B R^{a}-\Omega_{\mathrm{Kerr}} \varphi^{a}\right),
$$

where $\ell_{0}^{a}=T^{a}+R^{a}$, then the values of the lapse and shift fields at the horizon are given by

$$
\alpha=B, \quad \beta^{a}=\alpha R^{a}-\Omega_{\mathrm{Kerr}} \varphi^{a} .
$$


Abhay Ashtekar and Badri Krishnan

We are free to choose the lapse $\alpha$ arbitrarily on $S$, but this fixes the shift $\beta^{a}$. The tangential part of $\beta^{a}$ can, if desired, be chosen differently depending on the asymptotic value of $\beta^{a}$ at infinity which determines the angular velocity of inertial observers at infinity.

Next, one imposes the condition that the infinitesimal world-tube of apparent horizons is an 'instantaneous' non-expanding horizon. This requirement is equivalent to

$$
\mathcal{L}_{t} \tilde{q}_{a b}=0, \quad \mathcal{L}_{\varphi} \tilde{q}_{a b}=0
$$

on $\Delta$, which imply that the shear and expansion of $\ell^{a}$ vanish identically. These can be easily translated to conditions that the Cauchy data $\left(\bar{q}_{a b}, \bar{K}_{a b}\right)$ must satisfy at the horizon $[72,124]$. These boundary conditions are equivalent to the quasi-equilibrium boundary conditions developed by Cook [72]. However, the isolated horizon formalism allows greater control on the physical parameters of the black hole. In particular, we are not restricted just to the co-rotational or irrotational cases. Furthermore, the use of IHs streamlines the procedure: Rather than adding conditions one by one as needed, one begins with a physically well-motivated notion of horizons in equilibrium and systematically derives its consequences.

Up to this point, the considerations are general in the sense that they are not tied to a particular method of solving the initial value problem. However, for the quasi-equilibrium problem, it is the conformal thin-sandwich method [192, 70] that appears to be best suited. This approach is based on the conformal method $[144,191]$ where we write the 3 -metric as $\bar{q}_{a b}=\psi^{4} \hat{q}_{a b}$. The free data consists of the conformal 3-metric $\hat{q}_{a b}$, its time derivative $\mathcal{L}_{t} \hat{q}_{a b}$, the lapse $\alpha$, and the trace of the extrinsic curvature $\bar{K}$. Given this free data, the remaining quantities, namely the conformal factor and the shift, are determined by elliptic equations provided appropriate boundary conditions are specified for them on the horizon ${ }^{6}$. It turns out [124] that the horizon conditions (63) are well-tailored for this purpose. While the issue of existence and uniqueness of solutions using these boundary conditions has not been proven, it is often the case that numerical calculations are convergent and the resulting solutions are well behaved. Thus, these conditions might therefore be sufficient from a practical point of view.

In the above discussion, the free data consisted of $\left(\hat{q}_{a b}, \mathcal{L}_{t} \hat{q}_{a b}, \alpha, \bar{K}\right)$ and one solved elliptic equations for $\left(\psi, \beta^{a}\right)$. However, it is common to consider an enlarged initial value problem by taking $\mathcal{L}_{t} \bar{K}$ as part of the free data (usually set to zero) and solving an elliptic equation for $\alpha$. We now need to prescribe an additional boundary condition for $\alpha$. It turns out that this can be done by using WIHs, i.e., by bringing in surface gravity, which did not play any role so far. From the definition of surface gravity in Equations $(7,8)$, it is clear that the expression for $\kappa$ will involve a time derivative; in particular, it turns out to involve the time derivative of $\alpha$. It can be shown that by choosing $\mathcal{L}_{t} \alpha$ on $S$ (e.g., by taking $\mathcal{L}_{t} \alpha=0$ ) and requiring surface gravity to be constant on $S$ and equal to $\kappa_{\text {Kerr }}$, one obtains a suitable boundary condition for $\alpha$. (The freedom to choose freely the function $\mathcal{L}_{t} \alpha$ mirrors the fact that fixing surface gravity does not uniquely fix the rescaling freedom of the null normal.) Note that $\kappa$ is required to be constant only on $S$, not on $\Delta$. To ask it to be constant on $\Delta$ would require $\mathcal{L}_{t} \kappa=0$, which in turn would restrict the second time derivative; this necessarily involves the evolution equations, and they are not part of the initial data scheme.

One may imagine using the yet stronger notion of an $\mathrm{IH}$, to completely fix the value of the lapse at the horizon. But this requires solving an elliptic equation on the horizon and the relevant elliptic operator has a large kernel [16, 72]. Nonetheless, the class of initial data on which its inverse exists is infinite dimensional so that the method may be useful in practice. However, this condition would genuinely restrict the permissible initial data sets. In this sense, while the degree

\footnotetext{
${ }^{6}$ Cook's boundary condition on the conformal factor $\psi$ (Equation (82) in [72]) is equivalent to $\Theta_{(\ell)}=0$ which (in the co-rotating case, or more generally, when the 2-metric on $S$ is axi-symmetric) reduces to $\mathcal{L}_{t} \psi=0$ on $S$. The Yo et al. boundary condition on $\psi$ (Equation (48) of [190]) is equivalent to $\mathcal{L}_{\bar{t}} \psi=0$ on $S$, where, however, the evolution vector field $\bar{t}^{a}$ is obtained by a superposition of two Kerr-Schild data.
}

Living Reviews in Relativity

http://www . livingreviews . org/lrr-2004-10 
of isolation implied by the IH boundary condition is likely to be met in the asymptotic future, for quasi-equilibrium initial data it is too strong in general. It is the WIH boundary conditions that appear to be well-tailored for this application.

Finally, using methods introduced by Dain [80], a variation of the above procedure was recently introduced to establish the existence and uniqueness of solutions and to ensure that the conformal factor $\psi$ is everywhere positive [81]. One again imposes Equation (63). However, in place of Dirichlet boundary conditions (62) on the shift, one now imposes Neumann-type conditions on certain components of $\beta^{a}$. This method is expected to be applicable all initial data constructions relying on the conformal method. Furthermore, the result might also be of practical use in numerical constructions to ensure that the codes converge to a well behaved solution.

\subsubsection{Binding energy}

For initial data representing a binary black hole system, the quantity $E \mathrm{~b}=M_{\mathrm{ADM}}-M_{1}-M_{2}$ is called the effective binding energy, where $M_{\mathrm{ADM}}$ is the ADM mass, and $M_{1,2}$ are the individual masses of the two black holes. Heuristically, even in vacuum general relativity, one would expect $\mathrm{Eb}$ to have several components. First there is the analog of the Newtonian potential energy and the spin-spin interaction, both of which may be interpreted as contributing to the binding energy. But $E \mathrm{~b}$ also contains contributions from kinetic energy due to momentum and orbital angular momentum of black holes, and energy in the gravitational radiation in the initial data. It is only when these are negligible that $E \mathrm{~b}$ is a good measure of the physical binding energy.

The first calculation of binding energy was made by Brill and Lindquist in such a context. They considered two non-spinning black holes initially at rest [63]. For large separations, they found that, in a certain mathematical sense, the leading contribution to binding energy comes just from the usual Newtonian gravitational potential. More recently, Dain [79] has extended this calculation to the case of black holes with spin and has shown that the spin-spin interaction energy is correctly incorporated in the same sense.

In numerical relativity, the notion of binding energy has been used to locate sequences of quasi-circular orbits. The underlying heuristic idea is to minimize $E \mathrm{~b}$ with respect to the proper separation between the holes, keeping the physical parameters of the black holes fixed. The value of the separation which minimizes $E \mathrm{~b}$ provides an estimate of sizes of stable 'circular' orbits [71, $39,159]$. One finds that these orbits do not exist if the orbital angular momentum is smaller than a critical value (which depends on other parameters) and uses this fact to approximately locate the 'inner-most stable circular orbit' (ISCO). In another application, the binding energy has been used to compare different initial data sets which are meant to describe the same physical system. If the initial data sets have the same values of the black hole masses, angular momenta, linear momenta, orbital angular momenta, and relative separation, then any differences in $E \mathrm{~b}$ should be due only to the different radiation content. Therefore, minimization of $E \mathrm{~b}$ corresponds to minimization of the amount of radiation in the initial data [158].

In all these applications, it is important that the physical parameters of the black holes are calculated accurately. To illustrate the potential problems, let us return to the original Brill-Lindquist calculation [63]. The topology of the spatial slice is $\mathbb{R}^{3}$ with two points (called 'punctures') removed. These punctures do not represent curvature singularities. Rather, each of them represents a spatial infinity of an asymptotically flat region which is hidden behind an apparent horizon. This is a generalization of the familiar Einstein-Rosen bridge in the maximally extended Schwarzschild solution. The black hole masses $M_{1}$ and $M_{2}$ are taken to be the ADM masses of the corresponding hidden asymptotic regions. (Similarly, in [79], the angular momentum of each hole is defined to be the ADM angular momentum at the corresponding puncture.) Comparison between $E \mathrm{~b}$ and the Newtonian binding energy requires us to define the distance between the holes. This is taken to be the distance between the punctures in a fiducial flat background metric; the physical distance be- 
tween the two punctures is infinite since they represent asymptotic ends of the spatial 3-manifold. Therefore, the sense in which one recovers the Newtonian binding energy as the leading term is physically rather obscure.

Let us re-examine the procedure with an eye to extending it to a more general context. Let us begin with the definition of masses of individual holes which are taken to be the ADM masses in the respective asymptotic regions. How do we know that these are the physically appropriate quantities for calculating the potential energy? Furthermore, there exist initial data sets (e.g., Misner data $[150,151])$ in which each black hole does not have separate asymptotic regions; there are only two common asymptotic regions connected by two wormholes. For these cases, the use of ADM quantities is clearly inadequate. The same limitations apply to the assignment of angular momentum.

A natural way to resolve these conceptual issues is to let the horizons, rather than the punctures, represent black holes. Thus, in the spirit of the $\mathrm{IH}$ and $\mathrm{DH}$ frameworks, it is more appropriate to calculate the mass and angular momentum using expressions $(60,59)$ which involve the geometry of the two apparent horizons. (This requires the apparent horizons to be axi-symmetric, but this limitation could be overcome following the procedure suggested in Section 8.) Similarly, the physical distance between the black holes should be the smallest proper distance between the two apparent horizons. To test the viability of this approach, one can repeat the original Brill-Lindquist calculation in the limit when the black holes are far apart [136]. One first approximately locates the apparent horizon, finds the proper distance $d$ between them, and then calculates the horizon masses (and thereby $E_{\mathrm{b}}$ ) as a power series in $1 / d$. The leading-order term does turn out to be the usual Newtonian gravitational potential energy but the higher order terms are now different from [63]. Similarly, it would be interesting to repeat this for the case of spinning black holes and recover the leading order term of [79] within this more physical paradigm using, say, the BowenYork initial data. This result would re-enforce the physical ideas and the approach can then be used as a well defined method for calculating binding energy in more general situations.

\section{Remark}

In the examples mentioned above, we focussed on mass and angular momentum; linear momentum was not considered. A meaningful definition for the linear momentum of a black hole would be useful for several problems: It would enable one to define the orbital angular momentum, locate quasi-circular orbits and enable a comparison between different initial data sets. However, just as angular momentum is associated with rotational symmetry, linear momentum ought to be associated with a space translational symmetry. In a general curved space-time, one does not have even an approximate notion of translations except at infinity. Thus, whenever one speaks of linear momentum of initial data (e.g., in the BowenYork construction [57]) the quantity is taken to be the appropriate component of the ADM momentum at infinity. For reasons discussed above, it is more appropriate to associate black hole parameters with the respective horizons. So, a question naturally arises: Can one define linear momentum as an integral over the apparent horizon? If $z^{a}$ is a vector field on $M$ which, for some reason, can be regarded as an approximate translational symmetry, and $S$ the apparent horizon, then one might naively define the linear momentum associated with $z^{a}$ as

$$
P_{S}^{(z)}=\frac{1}{8 \pi} \oint_{S}\left(\bar{K}_{a b}-\bar{K} \bar{q}_{a b}\right) z^{a} R^{b} d^{2} S
$$

Unfortunately, this definition is not satisfactory. For example, in the boosted Kerr-Schild metric, if one lets $z^{a}$ to be the translational vector field of the background Minkowski space, Equation (64) does not yield the physically expected linear momentum of the boosted black hole. See [136] for further discussion and for other possible ways to define linear momentum. Further work is needed to address this question satisfactorily.

Living Reviews in Relativity

http://www. livingreviews . org//rr-2004-10 


\subsection{Black hole multipole moments}

Let us begin by considering the notion of source and field multipole moments in Newtonian gravity and in flat space electrodynamics. Field multipoles appear in the asymptotic expansions of the fields at infinity while the source multipoles are defined in terms of the mass or charge distribution of the source. These two sets of multipole moments are related to each other via field equations. The same is true in linearized general relativity [165]. Also, the well known quadrupole formula relates the rate of change of the quadrupole moment to the energy flux at infinity due to gravitational waves.

The situation in exact, non-linear general relativity is not so simple. Using the geometric structure of the gravitational field near spatial infinity, the field multipoles for stationary spacetimes were studied by Geroch, Hansen, Beig, Simon, and others [97, 107, 43, 41, 40, 42]. They found that, just as in electrodynamics, the gravitational field has two sets of multipoles: The mass multipoles $M_{n}$ and the angular momentum multipoles $J_{n}$. The knowledge of these multipole moments suffices to determine the space-time geometry in a neighborhood of spatial infinity [41, $40,42]$. Thus, at least in the context of stationary space-times, the field multipole moments are well understood. However, in problems involving equations of motion, it is the source multipoles that are of more direct interest. It is natural to ask if these can be defined for black holes.

The answer is affirmative for black holes in equilibrium, which can be represented by isolated horizons. For simplicity, we will consider only type II (i.e., axisymmetric), non-extremal isolated horizons in vacuum. The source multipoles are two sets $M_{n}$ and $J_{n}$ of numbers which provide a diffeomorphism invariant characterization of the horizon geometry.

As before, let $S$ be a cross-section of $\Delta$. We denote the intrinsic Riemannian metric on it by $\tilde{q}_{a b}$, the corresponding area 2 -form by $\tilde{\epsilon}_{a b}$, and the derivative operator by $\tilde{D}_{a}$. Since the horizon is of type II, there exists a vector field $\varphi^{a}$ on $S$ such that $\mathcal{L}_{\varphi} \tilde{q}_{a b}=0$. The two points where $\varphi^{a}$ vanishes are called the poles of $S$. The integral curves of $\varphi^{a}$ are natural candidates for the "lines of latitude' on $S$, and the lines of longitude are the curves which connect the two poles and are orthogonal to $\varphi^{a}$. This leads to an invariantly defined coordinate $\zeta \in[-1,1]-$ the analog of the function $\cos \theta$ in usual spherical coordinates - defined by

$$
\tilde{D}_{a} \zeta=\frac{1}{R^{2}} \tilde{\epsilon}_{b a} \varphi^{b},
$$

where $R$ is the area radius of $S$. The freedom of adding a constant to $\zeta$ is removed by requiring $\oint_{S} \zeta \tilde{\epsilon}=0$. With $\phi \in[0,2 \pi)$ being an affine parameter along $\varphi^{a}$, the 2-metric $\tilde{q}_{a b}$ takes the canonical form

$$
\tilde{q}_{a b}=R^{2}\left(f^{-1} \tilde{D}_{a} \zeta \tilde{D}_{b} \zeta+f \tilde{D}_{a} \phi \tilde{D}_{b} \phi\right),
$$

where $f=\varphi_{a} \varphi^{a} / R^{2}$. The only remaining freedom in the choice of coordinates is a rigid shift in $\phi$. Thus, in any axi-symmetric geometry, there is an invariantly defined coordinate $\zeta$, and multipole moments are defined using the Legendre polynomials in $\zeta$ as weight functions.

Recall from Section 2.1.3 that the invariant content in the geometry of an isolated horizon is coded in (the value of its area and) $\Psi_{2}$. The real part of $\Psi_{2}$ is proportional to the scalar curvature $\tilde{R}$ of $\tilde{q}_{a b}$ and captures distortions [98, 88], while the imaginary part of $\Psi_{2}$ yields the curl of $\omega_{a}$ and captures the angular momentum information. (The free function $f$ in Equation (66) determines and is completely determined by the scalar curvature $\tilde{R}$.) Multipoles are constructed directly from $\Psi_{2}$. The angular momentum multipoles are defined as

$$
J_{n}=-\sqrt{\frac{4 \pi}{2 n+1}} \frac{R_{\Delta}^{n+1}}{4 \pi G} \oint_{S} Y_{n}^{0}(\zeta) \operatorname{Im}\left[\Psi_{2}\right] d^{2} V,
$$

where $Y_{n}^{0}(\zeta)$ are the spherical harmonics. (Axi-symmetry ensures that $Y_{n}^{m}$ vanish if $m \neq 0$.) The normalization factors have been chosen to ensure that the dimensions of multipoles are the 
physically expected ones and $J_{1}$ is the angular momentum of the isolated horizon. The mass multipoles are defined similarly as the moments of $\tilde{R}$ :

$$
M_{n}=-\sqrt{\frac{4 \pi}{2 n+1}} \frac{M_{\Delta} R_{\Delta}^{n}}{2 \pi} \oint_{S} Y_{n}^{0}(\zeta) \operatorname{Re}\left[\Psi_{2}\right] d^{2} V,
$$

where the normalization factor has been chosen in order to ensure the correct physical dimensions and $M_{0}=M_{\Delta}$.

These multipoles have a number of physically desired properties:

- The angular momentum monopole vanishes, $J_{0}=0$. (This is because we are considering only smooth fields. When $\omega_{a}$ has a wire singularity similar to the Dirac monopole in electrodynamics, i.e., when the horizon has NUT charge, then $J_{0}$ would be non-zero.)

- The mass dipole vanishes, $M_{1}=0$. This tells us that we are in the rest frame of the horizon.

- For a type I (i.e., spherically symmetric) isolated horizon, $\operatorname{Im}\left[\Psi_{2}\right]=0$ and $\operatorname{Re}\left[\Psi_{2}\right]$ is constant. This implies that $M_{0}$ is the only non-zero multipole moment.

- If the horizon is symmetric under reflections as in the Kerr solution (i.e., $\Psi_{2} \mapsto \Psi_{2}^{\star}$ when $\zeta \mapsto-\zeta$ ), then $M_{n}$ vanishes for odd $n$ while $J_{n}$ vanishes for even $n$.

There is a one-one correspondence between the multipole moments $\left\{J_{n}, M_{n}\right\}$ and the geometry of the horizon: Given the horizon area $a_{\Delta}$ and multipoles $\left\{J_{n}, M_{n}\right\}$, assuming the multipoles satisfy a convergence condition for large $n$, we can reconstruct a non-extremal isolated horizon geometry $\left(\Delta, q_{a b}, \mathcal{D}_{a}\right)$, uniquely up to diffeomorphisms, such that the area of $\Delta$ is $a_{\Delta}$ and its multipole moments are the given $\left\{J_{n}, M_{n}\right\}$. In vacuum, stationary space-times, the multipole moments also suffice to determine the space-time geometry in the vicinity of the horizon. Thus, we see that the horizon multipole moments have the expected properties. In the extremal case, because of a surprising uniqueness result [143], the $M_{n}, J_{n}$ are universal - the same as those on the extremal Kerr IH and the 'true multipoles' which can distinguish one extremal IH from another are constructed using different fields in place of $\Psi_{2}$ [24]. Finally, note that there is no a-priori reason for these source multipoles to agree with the field multipoles at infinity; there could be matter fields or radiation outside the horizon which contribute to the field multipoles at infinity. The two sets of quantities need not agree even for stationary, vacuum space-times because of contributions from the gravitational field in the exterior region. For the Kerr space-time, the source and field moments are indeed different for $n \geq 2$. However, the difference is small for low $n$ [24].

See [24] for further discussion and for the inclusion of electromagnetic fields, and [48] for the numerical implementation of these results.

\subsection{Waveform extraction}

The prospect of receiving gravitational waves offers exciting opportunities for astrophysics and for testing the dynamical, strong field regime of general relativity (see, e.g., [78]). Black holes are among the most promising sources both for terrestrial and space-based observatories. Extracting gravitational waveform resulting from, say, gravitational collapse [153] or black hole mergers [138] is one of the important goals of numerical relativity. The eventual aim is to provide waveforms which, in conjunction with gravitational wave detectors, can be used to study the astrophysics of these gravitational wave sources. In this section, we summarize a method of waveform extraction based on the isolated horizon formalism.

The theory of gravitational radiation in exact general relativity is based on structures defined at future null infinity $\mathcal{I}^{+}$. In particular, associated with every cross-section of $\mathcal{I}^{+}$- which represents

Living Reviews in Relativity

http://www . livingreviews . org//rr-2004-10 
a retarded 'instant of time' - there is a well defined notion of mass, introduced by Bondi [54], which decreases as gravitational radiation flows across $\mathcal{I}^{+}$. On the other hand, except for those based on conformal methods, most simulations only deal with a finite portion of space-time and thus have no direct access to $\mathcal{I}^{+}$. Instead, one usually uses scalars such as the Weyl tensor component $\Psi_{4}$ to define the radiation waveform. However, $\Psi_{4}$ depends on the choice of a null tetrad as well as coordinates. While a natural tetrad is available for the perturbation theory on Kerr back ground, this is not true in general. In this section we will sketch an approach to solve both these problems using the isolated horizon framework: One can construct an approximate analog of $\mathcal{I}^{+}$ for a suitable, finite portion of space-time, and introduce a geometrically defined null tetrad and coordinates to extract gauge invariant, radiative information from simulations.

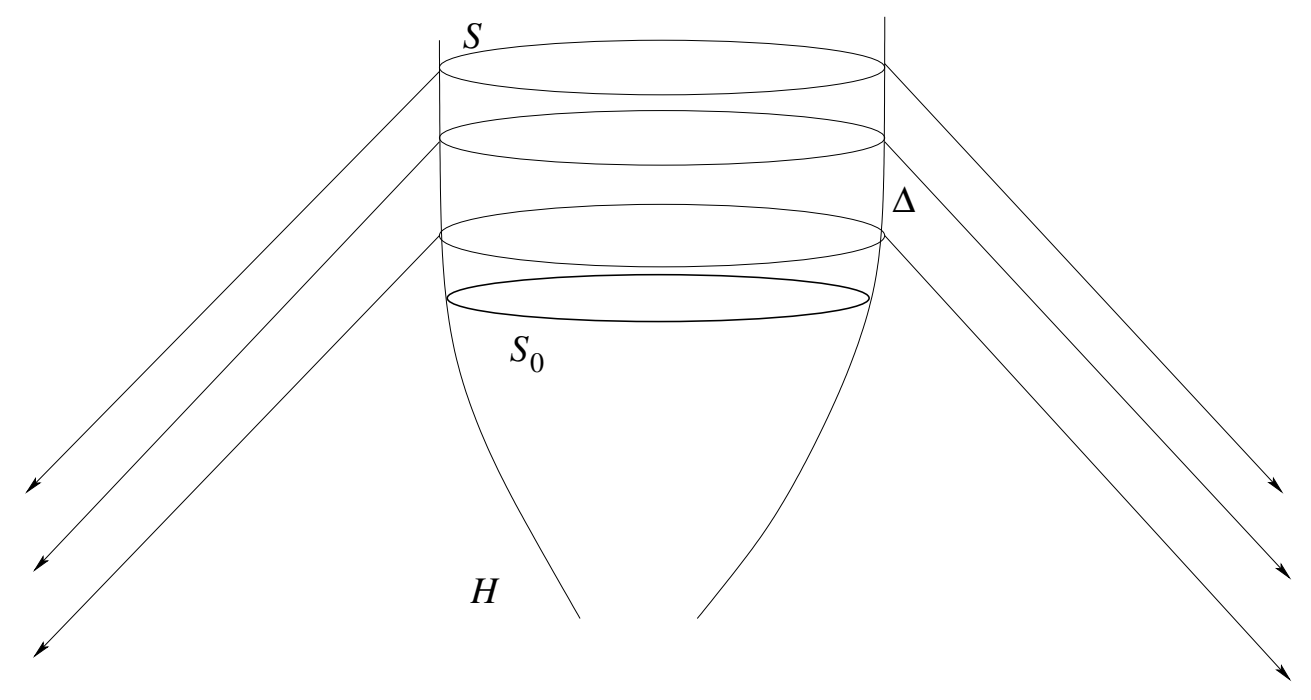

Figure 8: Bondi-like coordinates in a neighborhood of $\Delta$.

Our procedure is analogous to the one used at null infinity to construct the Bondi-type coordinates starting from $\mathcal{I}^{+}$. Let us assume that $(\Delta,[\ell])$ is an $\mathrm{IH}$ and consider its preferred foliation (i.e., the "rest frame" of the horizon) defined in Section 2.1.3. Using terminology from null infinity, we will refer to the leaves of the foliation as the good cuts of $\Delta$. Pick a null normal $\ell^{a}$ from the $\left[\ell^{a}\right]$; this can be done, e.g., by choosing a specific value for surface gravity. Let $n_{a}$ be the unique 1 -form satisfying $\ell^{a} n_{a}=-1$ and which is orthogonal to the good cuts. Let $(v, \theta, \phi)$ be coordinates on $\Delta$ such that $v$ is an affine parameter along $\ell^{a}$, and the good cuts are given by surfaces of constant $v$. Here $\theta$ and $\phi$ are coordinates on the good cut satisfying $\mathcal{L}_{\ell} \theta=0$ and $\mathcal{L}_{\ell} \phi=0$. Next, consider past null geodesics emanating from the good cuts with $-n^{a}$ as their initial tangent vector. Let the null geodesics be affinely parameterized and let the affine parameter be called $r$ and set $r=r_{0}$ on $\Delta$. Lie drag the coordinates $(v, \theta, \phi)$ along null geodesics. This leads to a set of coordinates $(r, v, \theta, \phi)$ in a neighborhood of the horizon. The only arbitrariness in this coordinate system is in the choice of $(\theta, \phi)$ on one good cut and the choice of a number $r_{0}$. Using vacuum Einstein's equations, one can obtain a systematic expansion of the metric in inverse powers of $\left(r-r_{0}\right)$ [12].

One can also define a null tetrad in the neighborhood in a similar fashion. Let $m^{a}$ be an arbitrary complex vector tangent to the good cuts such that $(\ell, n, m, \bar{m})$ is a null tetrad on $\Delta$. Using parallel transport along the null geodesics, we can define a null tetrad in the neighborhood. This tetrad is unique up to the spin rotations of $m: m \rightarrow e^{2 i \theta} m$ on the fiducial good cut. This construction is shown in Figure 8. The domain of validity of the coordinate system and tetrads is the space-time region in which the null geodesics emanating from good cuts do not cross. 
Numerically, it might be possible to adapt existing event horizon finders to locate the outgoing past null cone of a cross-section of the horizon. This is because event horizon trackers also track null surfaces backward in time [82, 83]; the event horizon is the ingoing past null surface starting from sufficiently close to future timelike infinity while here we are interested in the outgoing past directed null surface starting from an apparent horizon.

In the Schwarzschild solution, it can be shown analytically that this coordinate system covers an entire asymptotic region. In the Kerr space-time, the domain of validity is not explicitly known, but in a numerical implementation, this procedure does not encounter geodesic crossing in the region of interest to the simulation [82]. In general space-times, the extraction of wave forms requires the construction to go through only along the past light cones of good cuts which lie in the distant future, whence problems with geodesic-crossing are unlikely to prevent one from covering a sufficiently large region with these coordinates. This invariantly defined structure provides a new approach to extract waveforms. First, the null tetrad presented above can be used to calculate $\Psi_{4}$. There is only a phase factor ambiguity (which is a function independent of $v$ and $r$ ) inherited from the ambiguity in the choice of $m^{a}$ on the fiducial good cut. Second, the past null cone of a good cut at a sufficiently late time can be used as an approximate null infinity. This should enable one to calculate dynamical quantities such as the analogs of the Bondi mass and the rate of energy loss from the black hole, now on the 'approximate' $\mathcal{I}^{+}$. However, a detailed framework to extract the approximate expressions for fluxes of energy and Bondi mass, with sufficient control on the errors, is yet to be developed. This is a very interesting analytical problem since its solution would provide numerical relativists with an algorithm to extract waveforms and fluxes of energy in gravitational waves in an invariant and physically reliable manner. Finally, the invariant coordinates and tetrads also enable one to compare late time results of distinct numerical simulations.

Living Reviews in Relativity

http://www . livingreviews . org/lrr-2004-10 


\section{Applications in Mathematical Physics}

For simplicity, in Section 4 we restricted our review of the laws of black hole mechanics to the Einstein-Maxwell theory. However, there is a large body of literature on black holes in more general theories with dilatonic, Yang-Mills, Higgs, Proca, and Skyrme fields. These fields are not expected to be physically significant in the macroscopic, astrophysical world. However, they are of considerable interest from a mathematical physics perspective because their inclusion brings about qualitative, structural changes in the theory. The most dramatic of these is that the uniqueness theorems that play a central role in the Einstein-Maxwell theory are no longer valid. Consequently, the structure of these 'colored' or 'hairy' black-holes is much more complicated than those in the Einstein-Maxwell theory, and most of the work in this area has been carried out through a combination of analytic and numerical methods. By now a very large body of facts about stationary black holes with hair has accumulated. A major challenge is to unify this knowledge through a few, general principles.

The isolated horizon framework has provided surprising insights into the properties of hairy black holes in equilibrium $[19,77,75,26,21,76]$. While the zeroth and first laws go through in a straightforward manner, the notion of the horizon mass now becomes much more subtle and its properties have interesting consequences. The framework also suggests a new phenomenological model of colored black holes as bound states of ordinary, uncolored black holes and solitons. This model successfully explains the qualitative behavior of these black holes, including their stability and instability, and provides unexpected quantitative relations between colored black holes and their solitonic analogs.

In these theories, matter fields are minimally coupled to gravity. If one allows non-minimal couplings, the first law itself is modified in a striking fashion: Entropy is no longer given by the horizon area but depends also on the matter fields. For globally stationary space-times admitting bifurcate Killing horizons, this result was first established by Jacobson, Kang, and Myers [123], and by Iyer and Wald $[183,184]$ for a general class of theories. For scalar fields non-minimally coupled to gravity, it has been generalized in the setting of Type II WIHs [22]. While the procedure does involve certain technical subtleties, the overall strategy is identical to that summarized in Section 4.1. Therefore we will not review this issue in detail.

This section is divided into two parts. In the first, we discuss the mechanics of weakly isolated horizons in presence of dilatons and Yang-Mills fields. In the second, we discuss three applications. This entire discussion is in the framework of isolated horizons because the effects of these fields on black hole dynamics remain largely unexplored.

\subsection{Beyond Einstein-Maxwell theory}

Our primary purpose in this section is to illustrate the differences from the Einstein-Maxwell theory. These stem from 'internal charges' and other 'quantum numbers' that are unrelated to angular momentum. Therefore, for simplicity, we will restrict ourselves to non-rotating weakly isolated horizons. Extension to include angular momentum is rather straightforward.

\subsubsection{Dilatonic couplings}

In dilaton gravity, the Einstein-Maxwell theory is supplemented with a scalar field - called the dilaton - and (in the Einstein frame) the Maxwell part of the action is replaced by

$$
S_{\mathrm{dil}}(\phi, A)=-\frac{1}{16 \pi} \int_{\mathcal{M}}\left[2(\nabla \phi)^{2}+e^{-2 \alpha \phi} \mathbf{F}_{a b} \mathbf{F}^{a b}\right], d^{4} v,
$$

where $\alpha \geq 0$ is a free parameter which governs the strength of the coupling of $\phi$ to the Maxwell field $\mathbf{F}_{a b}$. If $\alpha=0$, one recovers the standard Einstein-Maxwell-Klein-Gordon system, while $\alpha=1$ 
occurs in a low energy limit of string theory. For our illustrative purposes, it will suffice to consider the $\alpha=1$ case.

At spatial infinity, one now has three charges: the ADM mass $M_{\mathrm{ADM}}$, the usual electric charge $Q_{\infty}$, and another charge $\tilde{Q}_{\infty}$ :

$$
Q_{\infty}=\frac{1}{4 \pi} \oint_{S_{\infty}}^{\star} \mathbf{F}, \quad \tilde{Q}_{\infty}=\frac{1}{4 \pi} \oint_{S_{\infty}} e^{-2 \phi \star} \mathbf{F} .
$$

$\tilde{Q}_{\infty}$ is conserved in space-time (i.e., its value does not change if the 2 -sphere of integration is deformed) while $Q_{\infty}$ is not. From the perspective of weakly isolated horizons, it is more useful to use $a_{\Delta}, Q_{\Delta}$, and $\tilde{Q}_{\Delta}$ as the basic charges:

$$
Q_{\Delta}=\frac{1}{4 \pi} \oint_{S}^{\star} \mathbf{F}, \quad \tilde{Q}_{\Delta}=\frac{1}{4 \pi} \oint_{S} e^{-2 \phi \star} \mathbf{F},
$$

where $S$ is any cross-section of $\Delta$. Although the standard electric charge is not conserved in space-time, it is conserved along $\Delta$ whence $Q_{\Delta}$ is well-defined.

It is straightforward to extend the Hamiltonian framework of Section 4.1 to include the dilaton. To define energy, one can again seek live time-translation vector fields $t^{a}$, evolution along which is Hamiltonian. The necessary and sufficient condition now becomes the following: There should exist a phase space function $E_{\Delta}^{t}$, constructed from horizon fields, such that

$$
\delta E_{\Delta}^{t}=\frac{\kappa_{(t)}}{8 \pi G} \delta a_{\Delta}+\Phi_{(t)} \delta \tilde{Q}_{\Delta}
$$

Thus, the only difference from the Einstein-Maxwell case is that $Q_{\Delta}$ is now replaced by $\tilde{Q}_{\Delta}$. Again, there exists an infinite number of such live vector fields and one can construct them systematically starting with any (suitably regular) function $\kappa_{0}\left(a_{\Delta}, \tilde{Q}_{\Delta}\right)$ of the horizon area and charge and requiring $\kappa_{(t)}$ should equal $\kappa_{0}$.

The major difference arises in the next step, when one attempts to construct a preferred $t_{0}^{a}$. With the dilatonic coupling, the theory has a unique three parameter family of static solutions which can be labelled by $\left(a_{\Delta}, Q_{\Delta}, \tilde{Q}_{\Delta}\right)[101,95,96,148]$. As in the Reissner Nordström family, these solutions are spherically symmetric. In terms of these parameters, the surface gravity $\kappa_{(\xi)}$ of the static Killing field $\xi^{a}$, which is unit at infinity, is given by

$$
\kappa_{(\xi)}=\frac{1}{2 R_{\Delta}}\left(1+2 G \frac{Q_{\Delta} \tilde{Q}_{\Delta}}{R_{\Delta}^{2}}\right)\left(1-2 G \frac{Q_{\Delta} \tilde{Q}_{\Delta}}{R_{\Delta}^{2}}\right)^{-\frac{1}{2}}
$$

The problem in the construction of the preferred $t_{0}^{a}$ is that we need a function $\kappa_{0}$ which depends only on $a_{\Delta}$ and $\tilde{Q}_{\Delta}$ and, since $\kappa_{(\xi)}$ depends on all three horizon parameters, one can no longer set $\kappa_{0}=\kappa_{(\xi)}$ on the entire phase space. Thus, there is no live vector field $t_{0}^{a}$ which can generate a Hamiltonian evolution and agree with the time-translation Killing field in all static solutions. It was the availability of such live vector fields that provided a canonical notion of the horizon mass in the Einstein-Maxwell theory in Section 4.1.3.

One can weaken the requirements by working on sectors of phase space with fixed values of $Q_{\Delta}$. On each sector, $\kappa_{(\xi)}$ trivially depends only on $a_{\Delta}$ and $\tilde{Q}_{\Delta}$. So one can set $\kappa_{0}=\kappa_{(\xi)}$, select a canonical $t_{0}$, and obtain a mass function $M_{\Delta}=E_{\Delta}^{t_{0}}$. However, now the first law (72) is satisfied only if the variation is restricted such that $\delta Q_{\Delta}=0$. For general variation, one has the modified law [26]

$$
\delta M_{\Delta}=\frac{\kappa}{8 \pi G} \delta a_{\Delta}+\hat{\Phi} \delta \hat{Q}_{\Delta},
$$

where $\kappa=\kappa_{\left(t_{0}\right)}, \hat{\Phi}^{2}=\left(Q_{\Delta} \tilde{Q}_{\Delta} / R_{\Delta}^{2}\right)$ and $\hat{Q}_{\Delta}^{2}=Q_{\Delta} \tilde{Q}_{\Delta}$. Thus, although there is still a first law in terms of $t_{0}^{a}$ and $M_{\Delta}$, it does not have the canonical form (72) because $t_{0}^{a}$ does not generate

Living Reviews in Relativity

http: //www. livingreviews. org/Irr-2004-10 
Hamiltonian evolution on the entire phase space. More generally, in theories with multiple scalar fields, if one focuses only on static sectors, one obtains similar 'non-standard' forms of the first law with new 'work terms' involving scalar fields [100]. From the restricted perspective of static sector, this is just a fact. The isolated horizon framework provides a deeper underlying reason: In these theories, there is no evolution vector field $t^{a}$ defined for all points of the phase space, which coincides with the properly normalized Killing field on all static solutions, and evolution along which is Hamiltonian on the full phase space.

\subsubsection{Yang-Mills fields}

In the Einstein-Maxwell theory, with and without the dilaton, one can not construct a quantity with the dimensions of mass from the fundamental constants in the theory. The situation is different for Einstein-Yang-Mills theory because the coupling constant $g$ has dimensions $(L M)^{-1 / 2}$. The existence of such a dimensionful quantity has interesting consequences.

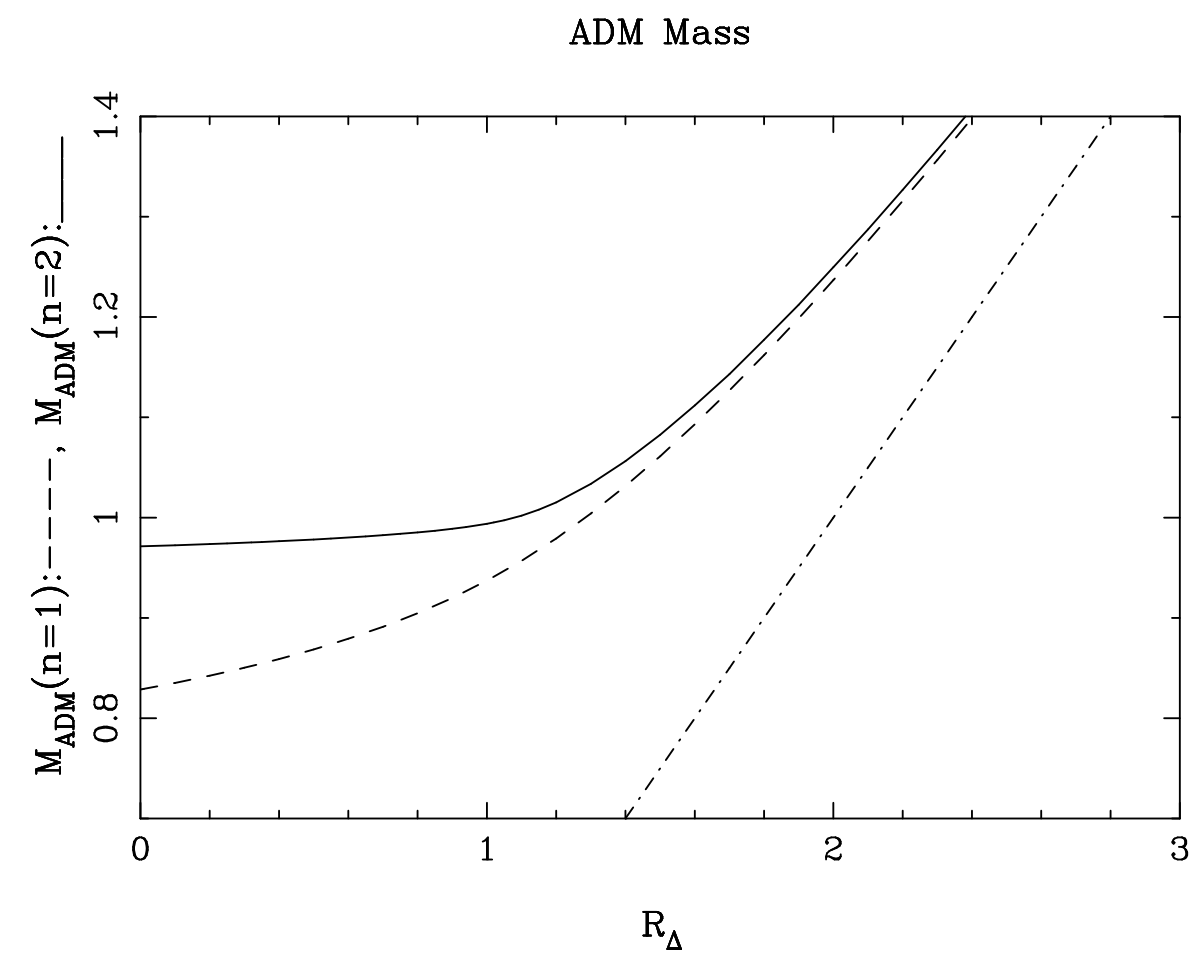

Figure 9: The ADM mass as a function of the horizon radius $R_{\Delta}$ of static spherically symmetric solutions to the Einstein-Yang-Mills system (in units provided by the Yang-Mills coupling constant). Numerical plots for the colorless $(n=0)$ and families of colored black holes $(n=1,2)$ are shown. (Note that the $y$-axis begins at $M=0.7$ rather than at $M=0$.)

For simplicity, we will restrict ourselves to SU(2) Yang-Mills fields, but results based on the isolated horizon framework go through for general compact groups [26]. Let us begin with a summary of the known static solutions. First, the Reissner-Nordström family constitutes a continuous 2-parameter set of static solutions of the Einstein-Yang-Mills theory, labelled by $\left(a_{\Delta}, Q_{\Delta}^{\mathrm{YM}}\right)$. In addition, there is a 1-parameter family of 'embedded Abelian solutions' with (a fixed) magnetic charge $P_{\Delta}^{0}$, labelled by $\left(a_{\Delta}, P_{\Delta}^{0}\right)$. Finally, there are families of 'genuinely non-Abelian solutions'. For these, the analog of the Israel theorem for Einstein-Maxwell theory fails to hold [127, 128, 129]; 
Abhay Ashtekar and Badri Krishnan

the theory admits static solutions which need not be spherically symmetric. In particular, an infinite family of solutions labelled by two integers $\left(n_{1}, n_{2}\right)$ is known to exist. All static, spherically symmetric solutions are known and they correspond to the infinite sub-family $\left(n_{1}, n_{2}=0\right)$, labelled by a single integer. However, the two parameter family is obtained using a specific ansatz, and other static solutions also exist. Although the available information on the static sector is quite rich, in contrast to the Einstein-Maxwell-dilaton system, one is still rather far from having complete control.

However, the existing results are already sufficient to show that, in contrast to the situation in the Einstein-Maxwell theory, the ADM mass is not a good measure of the black hole (or horizon) mass even in the static case. Let us consider the simplest case, the spherically symmetric static solutions labelled by a single integer $n$ (see Figure 9). Let us decrease the horizon area along any branch $n \neq 0$. In the zero area limit, the solution is known to converge point-wise to a regular, static, spherical solution, representing an Einstein-Yang-Mills soliton [38, 171, 60]. This solution has, of course, a non-zero ADM mass $M_{\mathrm{ADM}}^{\mathrm{sol}}$, which equals the limiting value of $M_{\mathrm{ADM}}^{\mathrm{BH}}$. However, in this limit, there is no black hole at all! Hence, this limiting value of the ADM mass can not be meaningfully identified with any horizon mass. By continuity, then, $M_{\mathrm{ADM}}^{\mathrm{BH}}$ can not be taken as an accurate measure of the horizon mass for any black hole along an $n \neq 0$ branch. Using the isolated horizon framework, it is possible to introduce a meaningful definition of the horizon mass on any given static branch.

To establish laws of black hole mechanics, one begins with appropriate boundary conditions. In the Maxwell case, the gauge freedom in the vector potential is restricted on the horizon by requiring $\mathcal{L}_{\ell} A_{a}=0$ on $\Delta$. The analogous condition ensuring that the Yang-Mills potential $\mathbf{A}$ is in an 'adapted gauge' on $\Delta$ is more subtle [26]. However, it does exist and again ensures that (i) the action principle is well defined, and (ii) the Yang-Mills electric potential $\boldsymbol{\Phi}_{(\ell)}:=-|\ell \cdot \mathbf{A}|$ is constant on the horizon, where the absolute sign stands for the norm in the internal space. The rest of the boundary conditions are the same as in Section 2.1.1. The proof of the zeroth law and the construction of the phase space is now straightforward. There is a well-defined notion of conserved horizon charges

$$
Q_{\Delta}^{\mathrm{YM}}:=-\left.\frac{1}{4 \pi} \oint_{S}\right|^{\star} \mathbf{F}\left|d^{2} V, \quad P_{\Delta}^{\mathrm{YM}}:=-\frac{1}{4 \pi} \oint_{S}\right| \mathbf{F} \mid d^{2} V,
$$

where $|\mathbf{F}|:=\left[\left(\epsilon^{a b} \mathbf{F}_{a b}^{i}\right)\left(\epsilon^{a b} \mathbf{F}_{a b}^{j}\right) K_{i j}\right]^{\frac{1}{2}}$, with $K_{i j}$ being the Cartan-Killing metric on SU(2), $\epsilon^{a b}$ the alternating tensor on the cross-section $S$ of $\Delta$, and where $\left|{ }^{\star} \mathbf{F}\right|$ is defined by replacing $\mathbf{F}$ with ${ }^{\star} \mathbf{F}$. Finally, one can again introduce live vector fields $t^{a}$, time evolution along which generates a Hamiltonian flow on the phase space, and establish a first law for each of these $t^{a}$ :

$$
\delta E_{\Delta}^{t}=\frac{1}{8 \pi G} \kappa_{(t)} \delta a_{\Delta}+\boldsymbol{\Phi}_{(t)} \delta Q_{\Delta}^{\mathrm{YM}} .
$$

Note that, even though the magnetic charge $P_{\Delta}^{\mathrm{YM}}$ is in general non-zero, it does not enter the statement of the first law. In the Abelian case, a non-zero magnetic charge requires non-trivial U(1) bundles, and Chern numbers characterizing these bundles are discrete. Hence the magnetic charge is quantized and, if the phase space is constructed from connections, $\delta P^{\mathrm{EM}}$ vanishes identically for any variation $\delta$. In the non-Abelian case, one can work with a trivial bundle and have non-zero $P_{\Delta}^{\mathrm{YM}}$. Therefore, $\delta P_{\Delta}^{Y M}$ does not automatically vanish and absence of this term in the first law is somewhat surprising.

A more significant difference from the Abelian case is that, because the uniqueness theorem fails, one can not use the static solutions to introduce a canonical function $\kappa_{0}$ on the entire phase space, whence as in the dilatonic case, there is no longer a canonical horizon mass $M_{\Delta}$ function on the entire phase space. In the next Section 6.2 we will see that it is nonetheless possible to introduce an extremely useful notion of the horizon mass for each static sequence.

Living Reviews in Relativity

http://www . livingreviews . org//rr-2004-10 


\subsection{Structure of colored, static black holes}

We will briefly summarize research in three areas in which the isolated horizon framework has been used to illuminate the structure of static, colored black holes and associated solitons ${ }^{7}$.

\subsubsection{Horizon mass}

Let us begin with Einstein-Yang-Mills theory considered in the last Section 6.1. As we saw, the ADM mass fails to be a good measure of the horizon mass for colored black holes. The failure of black hole uniqueness theorems also prevents the isolated horizon framework from providing a canonical notion of horizon mass on the full phase space. However, one can repeat the strategy used for dilatonic black holes to define horizon mass unambiguously for the static solutions [77, 26].

Consider a connected component of the known static solutions, labelled by $\vec{n}_{0} \equiv\left(n_{1}^{0}, n_{2}^{0}\right)$. Using for $\kappa_{0}$ the surface gravity of the properly normalized static Killing vector, in this sector one can construct a live vector field $t_{0}^{a}$ and obtain a first law. The energy $E_{\Delta}^{t_{0}}$ is well-defined on the full phase space and can be naturally interpreted as the horizon mass $M_{\Delta}^{\left(\vec{n}_{0}\right)}$ for colored black holes with 'quantum number' $\vec{n}_{0}$. The explicit expression is given by

$$
M_{\Delta}^{\left(\vec{n}_{0}\right)}=\frac{1}{2 G} \int_{0}^{R_{\Delta}} \beta_{\vec{n}_{0}}(R) d R
$$

where, following a convention in the literature on hairy black holes [77], we have used

$$
\beta_{\vec{n}_{0}}(R)=2\left[\kappa_{\vec{n}_{0}}(R)\right] R
$$

rather than the surface gravity $\kappa_{\vec{n}_{0}}(R)$ of static solutions. Now, as in the Einstein-Maxwell case, the Hamiltonian generating evolution along $t_{0}^{a}$ contains only surface terms: $H_{t_{0}}=E_{\mathrm{ADM}}^{t_{0}}-E_{\Delta}^{t_{0}}$ and is constant on each connected, static sector if $t_{0}^{a}$ coincides with the static Killing field on that sector. By construction, our $t_{0}^{a}$ has this property for the $\vec{n}_{0}$-sector under consideration. Now, in the Einstein-Maxwell case, since there is no constant with the dimension of energy, it follows that the restriction of $H_{\mathrm{ADM}}^{t_{0}}$ to the static sector must vanish. The situation is quite different in Einstein-Yang-Mills theory where the Yang-Mills coupling constant $g$ provides a scale. In $c=1$ units, $(g \sqrt{G})^{-1} \sim$ mass. Therefore, we can only conclude that

$$
M_{\mathrm{ADM}}^{(\vec{n})}=M_{\Delta}^{\left(\vec{n}_{0}\right)}+(g \sqrt{G})^{-1} C^{\left(\vec{n}_{0}\right)}
$$

for some $\vec{n}_{0}$-dependent constant $C^{\left(\vec{n}_{0}\right)}$. As the horizon radius shrinks to zero, the static solution $[127,181]$ under consideration tends to the solitonic solution with the same 'quantum numbers' $\vec{n}_{0}$. Hence, by taking this limit, we conclude $(g \sqrt{G})^{-1} C^{\left(\vec{n}_{0}\right)}=M_{\mathrm{ADM}}^{\text {sol, }\left(\vec{n}_{0}\right)}$. Therefore, on any $\vec{n}$-static sector, we have the following interesting relation between the black hole and solitonic solutions:

$$
M_{\mathrm{ADM}}^{\mathrm{BH},(\vec{n})}=\frac{1}{2 G} \int_{0}^{R_{\Delta}} \beta_{\vec{n}}(R) d R+M_{\mathrm{ADM}}^{\mathrm{sol},(\vec{n})}=M_{\Delta}^{\mathrm{BH},(\vec{n})}+M_{\mathrm{ADM}}^{\mathrm{sol},(\vec{n})}
$$

Thus, although the main motivation behind the isolated horizon framework was to go beyond globally time-independent situations, it has led to an interesting new relation between the ADM masses of black holes and their solitonic analogs already in the static sector.

The relation (80) was first proposed for spherical horizons in [77], verified in [75], and extended to distorted horizons in [26]. It provided impetus for new work by mathematical physicists working on colored black holes. The relation has been confirmed in three more general and non-trivial cases:

\footnotetext{
${ }^{7}$ We are grateful to Alejandro Corichi for correspondence on the recent results in this area.
} 
- Non-spherical, non-rotating black holes parameterized by two quantum numbers [132].

- Non-spherical solutions to the more general Einstein-Yang-Mills-Higgs theory [109], where distortions are caused by 'magnetic dipole hair' [130].

- Static solutions in the Born-Infeld theory [62].

\subsubsection{Phenomenological model of colored black holes}

Isolated horizon considerations suggested the following simple heuristic model of colored black holes [21]: A colored black hole with quantum numbers $\vec{n}$ should be thought of as 'bound states' of a ordinary (colorless) black hole and a soliton with color quantum numbers $\vec{n}$, where $\vec{n}$ can be more general than considered so far. Thus the idea is that an uncolored black hole is 'bare' and becomes 'colored' when 'dressed' by the soliton.

The mass formula (80) now suggests that the total ADM mass $M_{\mathrm{ADM}}^{(\vec{n})}$ has three components: the mass $M_{\Delta}^{(0)}$ of the bare horizon, the mass $M_{\mathrm{ADM}}^{\mathrm{sol}, \vec{n}}$ of the colored soliton, and a binding energy given by

$$
E_{\text {binding }}=M_{\Delta}^{(\vec{n})}\left(R_{\Delta}\right)-M_{\Delta}^{(0)}\left(R_{\Delta}\right) .
$$

If this picture is correct, being gravitational binding energy, $E_{\text {binding }}$ would have to be negative. This expectation is borne out in explicit examples. The model has several predictions on the qualitative behavior of the horizon mass (77), the surface gravity, and the relation between properties of black holes and solitons. We will illustrate these with just three examples (for a complete list with technical caveats, see [21]):

- For any fixed value of $R_{\Delta}$ and of all quantum numbers except $n$, the horizon mass and surface gravity decrease monotonically with $n$.

- For fixed values of all quantum numbers $\vec{n}$, the horizon mass $M_{\Delta}^{(\vec{n})}\left(R_{\Delta}\right)$ is non-negative, vanishing if and only if $R_{\Delta}$ vanishes, and increases monotonically with $R_{\Delta} \cdot \beta_{(\vec{n})}$ is positive and bounded above by 1 .

- For fixed $\vec{n}$, the binding energy decreases as the horizon area increases.

The predictions for fixed $\vec{n}$ have recently been verified beyond spherical symmetry: for the distorted, axially symmetric Einstein-Yang-Mills solutions in [132] and for the distorted 'dipole' solutions in Einstein-Yang-Mills-Higgs solutions in [109]. Taken together, the predictions of this model can account for all the qualitative features of the plots of the horizon mass and surface gravity as functions of the horizon radius and quantum numbers. More importantly, they have interesting implications on the stability properties of colored black holes.

One begins with an observation about solitons and deduces properties of black holes. EinsteinYang-Mills solitons are known to be unstable [173]; under small perturbations, the energy stored in the 'bound state' represented by the soliton is radiated away to future null infinity $\mathcal{I}^{+}$. The phenomenological model suggests that colored black holes should also be unstable and they should decay into ordinary black holes, the excess energy being radiated away to infinity. In general, however, even if one component of a bound system is unstable, the total system may still be stable if the binding energy is sufficiently large. An example is provided by the deuteron. However, an examination of energetics reveals that this is not the case for colored black holes, so instability should prevail. Furthermore, one can make a few predictions about the nature of instability. We summarize these for the simplest case of spherically symmetric, static black holes for which there is a single quantum number $n$ :

Living Reviews in Relativity

http: //www . livingreviews . org/Irr-2004-10 


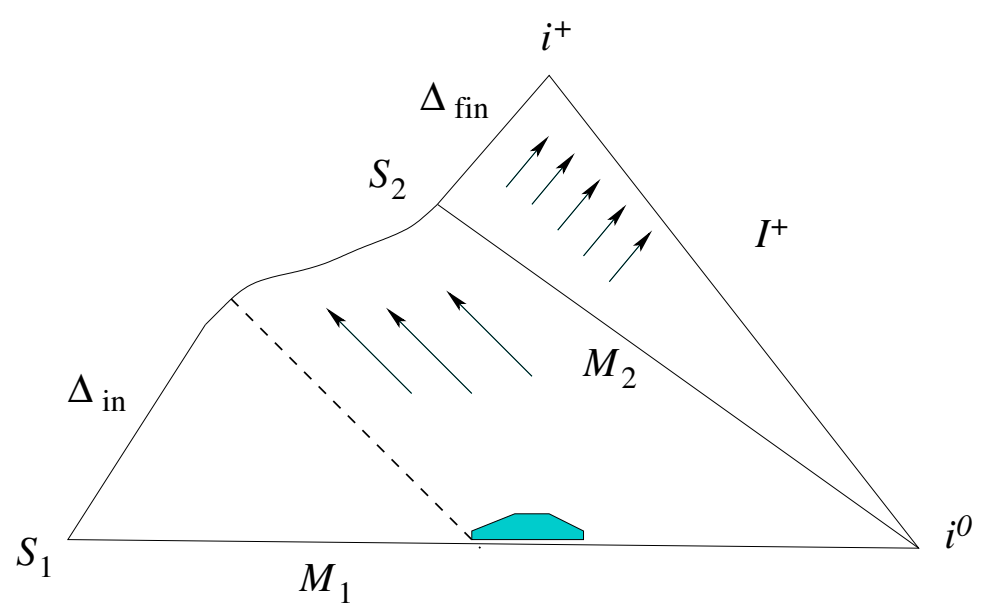

Figure 10: An initially static colored black hole with horizon $\Delta_{\text {in }}$ is slightly perturbed and decays to a Schwarzschild-like isolated horizon $\Delta_{\text {fin }}$, with radiation going out to future null infinity $\mathcal{I}^{+}$.

1. All the colored black holes on the $n$th branch have the same number (namely, $2 n$ ) of unstable modes as the $n$th soliton. (The detailed features of these unstable modes can differ especially because they are subject to different boundary conditions in the two cases.)

2. For a given $n$, colored black holes with larger horizon area are less unstable. For a given horizon area, colored black holes with higher value of $n$ are more unstable.

3. The 'available energy' for the process is given by

$$
E_{\text {available }}^{(n)}=M_{\text {sol }}^{(n)}-\left|E_{\text {binding }}^{\text {initial }}\right| .
$$

Part of it is absorbed by the black hole so that its horizon area increases and the rest is radiated away to infinity. Note that $E_{\text {available }}^{(n)}$ can be computed knowing just the initial configuration.

4. In the process the horizon area necessarily increases. Therefore, the energy radiated to infinity is strictly less than $E_{\text {available }}^{(n)}$.

Expectation 1 of the model is known to be correct [172]. Prediction 2 has been shown to be correct in the $n=1$, colored black holes in the sense that the frequency of all unstable modes is a decreasing function of the area, whence the characteristic decay time grows with area [181, 51]. To our knowledge a detailed analysis of instability, needed to test Predictions 3 and 4 are yet to be made.

Finally, the notion of horizon mass and the associated stability analysis has also provided an 'explanation' of the following fact which, at first sight, seems puzzling. Consider the 'embedded Abelian black holes' which are solutions to Einstein-Yang-Mills equations with a specific magnetic charge $P_{\Delta}^{0}$. They are isometric to a family of magnetically charged Reissner-Nordström solutions and the isometry maps the Maxwell field strength to the Yang-Mills field strength. The only difference is in the form of the connection; while the Yang-Mills potential is supported on a trivial $\mathrm{SU}(2)$ bundle, the Maxwell potential requires a non-trivial $\mathrm{U}(1)$ bundle. Therefore, it comes as an initial surprise that the solution is stable in the Einstein-Maxwell theory but unstable in the Einstein-Yang-Mills theory $[52,61]$. It turns out that this difference is naturally explained by the WIH framework. Since the solutions are isometric, their ADM mass is the same. However, since 
the horizon mass arises from Hamiltonian considerations, it is theory dependent: It is lower in the Einstein-Yang-Mills theory than in the Einstein-Maxwell theory! Thus, from the Einstein-YangMills perspective, part of the ADM mass is carried by the soliton and there is positive $E_{\text {available }}^{Y M}$ which can be radiated away to infinity. In the Einstein-Maxwell theory, $E_{\text {available }}^{\text {EM }}$ is zero. The stability analysis sketched above therefore implies that the solution should be unstable in the Einstein-Yang-Mills theory but stable in the Einstein-Maxwell theory. This is another striking example of the usefulness of the notion of the horizon mass.

\subsubsection{More general theories}

We will now briefly summarize the most interesting result obtained from this framework in more general theories. When one allows Higgs or Proca fields in addition to Yang-Mills, or considers Einstein-Skyrme theories, one acquires additional dimensionful constants which trigger new phenomena [50, 178, 181]. One of the most interesting is the 'crossing phenomena' of Figure 11 where curves in the 'phase diagram' (i.e., a plot of the ADM mass versus horizon radius) corresponding to the two distinct static families cross. This typically occurs in theories in which there is a length scale even in absence of gravity, i.e., even when Newton's constant is set equal to zero [154, 21].

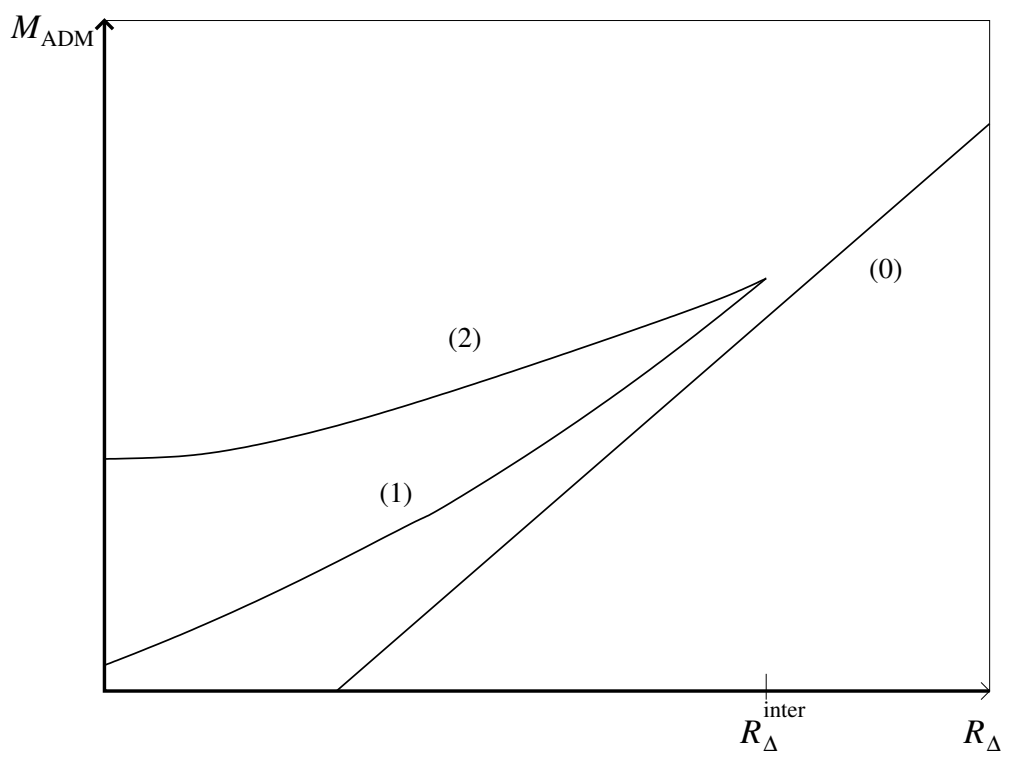

Figure 11: The ADM mass as a function of the horizon radius $R_{\Delta}$ in theories with a built-in non-gravitational length scale. The schematic plot shows crossing of families labelled by $n=1$ and $n=2$ at $R_{\Delta}=R_{\Delta}^{\text {inter }}$.

In this case, the notion of the horizon mass acquires further subtleties. If, as in the EinsteinYang-Mills theory considered earlier, families of static solutions carrying distinct quantum numbers do not cross, there is a well-defined notion of horizon mass for each static solution, although, as the example of 'embedded Abelian solutions' shows, in general its value is theory dependent. When families cross, one can repeat the previous strategy and use Equation (77) to define a mass $M_{\Delta}^{(n)}$ along each branch. However, at the intersection point $R_{\Delta}^{\text {inter }}$ of the $n$th and $(n+1)$ th branches, the mass is discontinuous. This discontinuity has an interesting implication. Consider the closed curve $C$ in the phase diagram, starting at the intersection point and moving along the $(n+1)$ th branch in the direction of decreasing area until the area becomes zero, then moving along $a_{\Delta}=0$

Living Reviews in Relativity

http: //www. livingreviews. org/Irr-2004-10 
to the $n$th branch and moving up to the intersection point along the $n$th branch (see Figure 11). Discontinuity in the horizon mass implies that the integral of $\beta\left(R_{\Delta}\right)$ along this closed curve is non-zero. Furthermore, the relation between the horizon and the soliton mass along each branch implies that the value of this integral has a direct physical interpretation:

$$
M_{\mathrm{sol}}^{(n+1)}-M_{\mathrm{sol}}^{(n)}=\frac{1}{2 G} \oint_{C} \beta(r) d r .
$$

This is a striking prediction because it relates differences in masses of solitons to the knowledge of horizon properties of the corresponding black holes! Because of certain continuity properties which hold as one approaches the static Einstein-Yang-Mills sector in the space to static solutions to Einstein-Yang-Mills-Higgs equations [76], one can also obtain a new formula for the ADM masses of Einstein-Yang-Mills solitons. If $\left(1-\beta_{(n)}(r)\right)$ for the black hole solutions of this theory is integrable over the entire positive half line, one has [21]:

$$
M_{\mathrm{sol}}^{(n)}=\frac{1}{2 G} \int_{0}^{\infty}\left(1-\beta_{(n)}(r)\right) d r .
$$

Both these predictions of the phenomenological model [21] have been verified numerically in the spherically symmetric case [76], but the axi-symmetric case is still open. 


\section{Applications in Quantum Gravity}

As discussed in the introduction, laws of black hole mechanics, discovered in the early seventies, provided a concrete challenge to candidate quantum theories of gravity: Account for the thermodynamic, black hole entropy through a detailed, statistical mechanical counting of appropriate micro-states. Indeed, this is essentially the only concrete quantitative hint we have had about the nature of quantum space-time geometry. The isolated horizon framework has been used to address this issue systematically and has led to the only available detailed calculations within a full-fledged approach to quantum gravity that encompass realistic black holes (which carry no or negligible gauge charges and may be distorted). As we will discuss in Section 8, what we know about dynamical horizons does suggest that there should be interesting generalizations of these results also to non-equilibrium situations. But so far there has been no work along these lines.

\subsection{Preliminaries}

The first and the second laws of black hole mechanics discussed in Section 4,

$$
\delta E=\frac{\kappa}{8 \pi G} \delta a+\text { work }, \quad d \delta a \geq 0,
$$

have a close similarity with the first and second laws of thermodynamics

$$
\delta E=T \delta S+\text { work }, \quad \delta S \geq 0 .
$$

This suggest that one should assign to black holes an entropy proportional to their area. Indeed, already in the early seventies, using imaginative thought experiments, Bekenstein [44, 45] argued that such an assignment is forced upon us if, in presence of black holes, we are not going to simply abandon the second law of thermodynamics. Specifically, consider an experiment in which a box containing radiation is slowly lowered into a black hole. At the end of the process, the entropy of the exterior region decreases in apparent violation of the second law. Bekenstein argued that the area of the horizon increases in the process so that the total entropy would not decrease if the black hole is assigned entropy equal to an appropriate multiple of the area. Hawking's celebrated calculation of black hole evaporation [112] provided the precise numerical coefficient. For, it suggested that we should assign the black hole a temperature $T=\hbar \kappa / 2 \pi$. By comparing the forms of the first laws of black hole mechanics and thermodynamics, one is then led to set

$$
S_{\mathrm{bh}}=\frac{a}{4 \ell_{\mathrm{Pl}}^{2}} \quad \text { with } \ell_{\mathrm{Pl}}^{2}=G \hbar .
$$

Note that the factor of $\hbar$ is absolutely essential: In the limit $\hbar \mapsto 0$, the black hole temperature goes to zero and its entropy tends to infinity just as one would expect classically.

The relation (87) is striking and deep because it brings together the three pillars of fundamental physics - general relativity, quantum theory, and statistical mechanics. However, the argument itself is a rather hodge-podge mixture of classical and semi-classical ideas, reminiscent of the Bohr theory of atom. A natural question then is: What is the analog of the more fundamental, PauliSchrödinger theory of the hydrogen atom? More precisely, what is the statistical mechanical origin of black hole entropy? To answer this question, one has to isolate the microscopic degrees of freedom responsible for this entropy. For a classical ideal gas, these are given by the positions and momenta of all molecules; for a magnet, by the states of each individual spin at lattice sites. How do we represent the analogous microscopic degrees of freedom for a black hole? They can not be described in terms of quantum states of physical gravitons because we are dealing with black holes in equilibrium. In the approach based on weakly isolated horizons, they are captured in the quantum states of the horizon geometry. Just as one would expect from Bekenstein's thought

Living Reviews in Relativity

http: //www . livingreviews . org/Irr-2004-10 
experiments, these degrees of freedom can interact with the exterior curved geometry, and the resulting notion of black hole entropy is tied to observers in the exterior regions.

A heuristic framework for the calculation of entropy was suggested by John Wheeler in the nineties, which he christened 'It from bit'. Divide the black hole horizon into elementary cells, each with one Planck unit of area $\ell_{\mathrm{Pl}}^{2}$ and assign to each cell two microstates. Then the total number of states $\mathcal{N}$ is given by $\mathcal{N}=2^{n}$, where $n=\left(a / \ell_{\mathrm{Pl}}^{2}\right)$ is the number of elementary cells, whence entropy is given by $S=\ln \mathcal{N} \sim a$. Thus, apart from a numerical coefficient, the entropy (' $I t$ ') is accounted for by assigning two states ('bit') to each elementary cell. This qualitative picture is simple and attractive. But it raises at least three central questions:

- What is the origin of the 'elementary cells' and why is each endowed with an area $\ell_{\mathrm{Pl}}^{2}$ ?

- What is the origin of the microstates carried by each elementary cell and why are there precisely two microstates?

- What does all this have to do with a black hole? Why doesn't it apply to any 2-surface, including a 2-sphere in Minkowski space-time?

An understanding of geometry of quantum WIHs provides a detailed framework which, in particular, answers these questions. The precise picture, as usual, is much more involved than that envisaged by Wheeler. Eigenvalues of area operator turn out to be discrete in quantum geometry and one can justify dividing the horizon 2-sphere into elementary cells. However, there are many permissible area eigenvalues and cells need not all carry the same area. To identify horizon surface states that are responsible for entropy, one has to crucially use the WIH boundary conditions. However, the number of surface states assigned to each cell is not restricted to two. Nonetheless, Wheeler's picture turns out to be qualitatively correct.

\subsection{Quantum horizon geometry}

For simplicity of presentation, in this section we will restrict ourselves to type I WIHs, i.e., the ones for which the only non-zero multipole moment is the mass monopole. The extension to include type II horizons with rotations and distortion will be briefly summarized in the next Section 7.3. Details can be found in $[10,11,20,8,24]$.

The point of departure is the classical Hamiltonian formulation for space-times $\mathcal{M}$ with a type I WIH $\Delta$ as an internal boundary, with fixed area $a_{0}$ and charges $Q_{0}^{\alpha}$, where $\alpha$ runs over the number of distinct charges (Maxwell, Yang-Mills, dilaton, ...) allowed in the theory. As we noted in Section 4.1, the phase space $\boldsymbol{\Gamma}$ can be constructed in a number of ways, which lead to equivalent Hamiltonian frameworks and first laws. However, so far, the only known way to carry out a background independent, non-perturbative quantization is through connection variables [32].

As in Figure 6 let us begin with a partial Cauchy surface $M$ whose internal boundary in $\mathcal{M}$ is a 2 -sphere cross-section $S$ of $\Delta$ and whose asymptotic boundary is a 2-sphere $S_{\infty}$ at spatial infinity. The configuration variable is an $\mathrm{SU}(2)$ connection $A_{a}^{i}$ on $M$, where $i$ takes values in the 3 -dimensional Lie-algebra $\mathrm{su}(2)$ of $\mathrm{SU}(2)$. Just as the standard derivative operator acts on tensor fields and enables one to parallel transport vectors, the derivative operator constructed from $A_{a}^{i}$ acts on fields with internal indices and enables one to parallel transport spinors. The conjugate momentum is represented by a vector field $P_{i}^{a}$ with density weight 1 which also takes values in $\mathrm{su}(2)$; it is the analog of the Yang-Mills electric field. (In absence of a background metric, momenta always carry a density weight 1.) $P_{i}^{a}$ can be regarded as a (density weighted) triad or a 'square-root' of the intrinsic metric $\tilde{q}_{a b}$ on $S:(8 \pi G \gamma)^{2} P_{i}^{a} P_{j}^{b} \delta_{i j}=\tilde{q} \tilde{q}^{a b}$, where $\delta_{i j}$ is the Cartan Killing metric on $\operatorname{su}(2), \tilde{q}$ is the determinant of $\tilde{q}_{a b}$ and $\gamma$ is a positive real number, called the Barbero-Immirzi parameter. This parameter arises because there is a freedom in adding to Palatini action a multiple 
of the term which is 'dual' to the standard one, which does not affect the equations of motion but changes the definition of momenta. This multiple is $\gamma$. The presence of $\gamma$ represents an ambiguity in quantization of geometry, analogous to the $\theta$-ambiguity in QCD. Just as the classical YangMills theory is insensitive to the value of $\theta$ but the quantum Yang-Mills theory has inequivalent $\theta$-sectors, classical relativity is insensitive to the value of $\gamma$ but the quantum geometries based on different values of $\gamma$ are (unitarily) inequivalent [94] (for details, see, e.g., [32]).

Thus, the gravitational part of the phase space $\boldsymbol{\Gamma}$ consists of pairs $\left(A_{a}^{i}, P_{i}^{a}\right)$ of fields on $M$ satisfying the boundary conditions discussed above. Had there been no internal boundary, the gravitational part of the symplectic structure would have had just the expected volume term:

$$
\boldsymbol{\Omega}_{V}\left(\delta_{1}, \delta_{2}\right)=\int_{M}\left(\delta_{2} A^{i} \wedge \delta_{1} \Sigma_{i}-\delta_{1} A^{i} \wedge \delta_{2} \Sigma_{i}\right)
$$

where $\Sigma_{a b i}:=\eta_{a b c} P_{i}^{c}$ is the 2 -form dual to the momentum $P_{i}^{c}$ and $\delta_{1}$, and $\delta_{2}$ denote any two tangent vectors to the phase space. However, the presence of the internal boundary changes the phase space-structure. The type I WIH conditions imply that the non-trivial information in the pull-back $A_{a}^{i}$ of $A_{a}^{i}$ to $S$ is contained in a U(1) connection $W_{a}:=A_{a}^{i} r_{i}$ on $S$, where $r^{i}$ is the unit, internal, radial vector field on $S$. Its curvature 2 -form $F_{a b}$ is related to the pull-back $\sum_{a b}^{i}$ of the 2 -form $\Sigma_{a b}^{i}$ to $S$ via

$$
F \equiv d W=-8 \pi G \gamma \frac{2 \pi}{a_{0}} \sum^{i} r_{i}
$$

The restriction is called the horizon boundary condition. In the Schwarzschild space-time, all the 2 -spheres on which it is satisfied lie on the horizon. Finally, the presence of the internal boundary modifies the symplectic structure: It now acquires an additional boundary term

$$
\boldsymbol{\Omega}\left(\delta_{1}, \delta_{2}\right)=\boldsymbol{\Omega}_{V}\left(\delta_{1}, \delta_{2}\right)+\boldsymbol{\Omega}_{S}\left(\delta_{1}, \delta_{2}\right)
$$

with

$$
\boldsymbol{\Omega}_{S}\left(\delta_{1}, \delta_{2}\right)=\frac{1}{2 \pi} \frac{a_{0}}{4 \pi G \gamma} \oint_{S} \delta_{1} W \wedge \delta_{2} W
$$

The new surface term is precisely the symplectic structure of a well-known topological field theory - the U(1)-Chern-Simons theory. The symplectic structures of the Maxwell, Yang-Mills, scalar, and dilatonic fields do not acquire surface terms. Conceptually, this is an important point: This, in essence, is the reason why (for minimally coupled matter) the black hole entropy depends just on the area and not, in addition, on the matter charges.

In absence of internal boundaries, the quantum theory has been well-understood since the mid-nineties (for recent reviews, see, [162, 176, 32]). The fundamental quantum excitations are represented by Wilson lines (i.e., holonomies) defined by the connection and are thus 1-dimensional, whence the resulting quantum geometry is polymer-like. These excitations can be regarded as flux lines of area for the following reason. Given any 2-surface $\mathbb{S}$ on $M$, there is a self-adjoint operator $\hat{A}_{\mathbb{S}}$ all of whose eigenvalues are known to be discrete. The simplest eigenvectors are represented by a single flux line, carrying a half-integer $j$ as a label, which intersects the surface $\mathbb{S}$ exactly once, and the corresponding eigenvalue $a_{\mathbb{S}}$ of $\hat{A}_{\mathbb{S}}$ is given by

$$
a_{\mathbb{S}}=8 \pi \gamma \ell_{\mathrm{Pl}}^{2} \sqrt{j(j+1)} .
$$

Thus, while the general form of the eigenvalues is the same in all $\gamma$-sectors of the quantum theory, their numerical values do depend on $\gamma$. Since the eigenvalues are distinct in different $\gamma$-sectors, it immediately follows that these sectors provide unitarily inequivalent representations of the algebra of geometric operators; there is 'super-selection'. Put differently, there is a quantization ambiguity, and which $\gamma$-sector is actually realized in Nature is an experimental question. One appropriate

Living Reviews in Relativity

http: //www . livingreviews . org/Irr-2004-10 
experiment, for example a measurement of the smallest non-zero area eigenvalue, would fix the value of $\gamma$ and hence the quantum theory. Every further experiment - e.g., the measurement of higher eigenvalues or eigenvalues of other operators such as those corresponding to the volume of a region - would provide tests of the theory. While such direct measurements are not feasible today we will see that, somewhat surprisingly, the Hawking-Bekenstein formula (87) for the entropy of large black holes provides a thought experiment to fix the value of $\gamma$.

Recall next that, because of the horizon internal boundary, the symplectic structure now has an additional surface term. In the classical theory, since all fields are smooth, values of fields on the horizon are completely determined by their values in the bulk. However, a key point about field theories is that their quantum states depend on fields which are arbitrarily discontinuous. Therefore, in quantum theory, a decoupling occurs between fields in the surface and those in the bulk, and independent surface degrees of freedom emerge. These describe the geometry of the quantum horizon and are responsible for entropy.

In quantum theory, then, it is natural to begin with a total Hilbert space $\mathcal{H}=\mathcal{H}_{V} \otimes \mathcal{H}_{S}$ where $\mathcal{H}_{V}$ is the well-understood bulk or volume Hilbert space with 'polymer-like excitations', and $\mathcal{H}_{S}$ is the surface Hilbert space of the U(1)-Chern-Simons theory. As depicted in Figure 12, the polymer excitations puncture the horizon. An excitation carrying a quantum number $j$ 'deposits' on $S$ an area equal to $8 \pi \ell_{\mathrm{Pl}}^{2} \sqrt{j(j+1)}$. These contributions add up to endow $S$ a total area $a_{0}$. The surface Chern-Simons theory is therefore defined on the punctured 2-sphere $S$. To incorporate the fact that the internal boundary $S$ is not arbitrary but comes from a WIH, we still need to incorporate the residual boundary condition (89). This key condition is taken over as an operator equation. Thus, in the quantum theory, neither the triad nor the curvature of $W$ are frozen at the horizon; neither is a classical field. Each is allowed to undergo quantum fluctuations, but the quantum horizon boundary condition requires that they have to fluctuate in tandem.

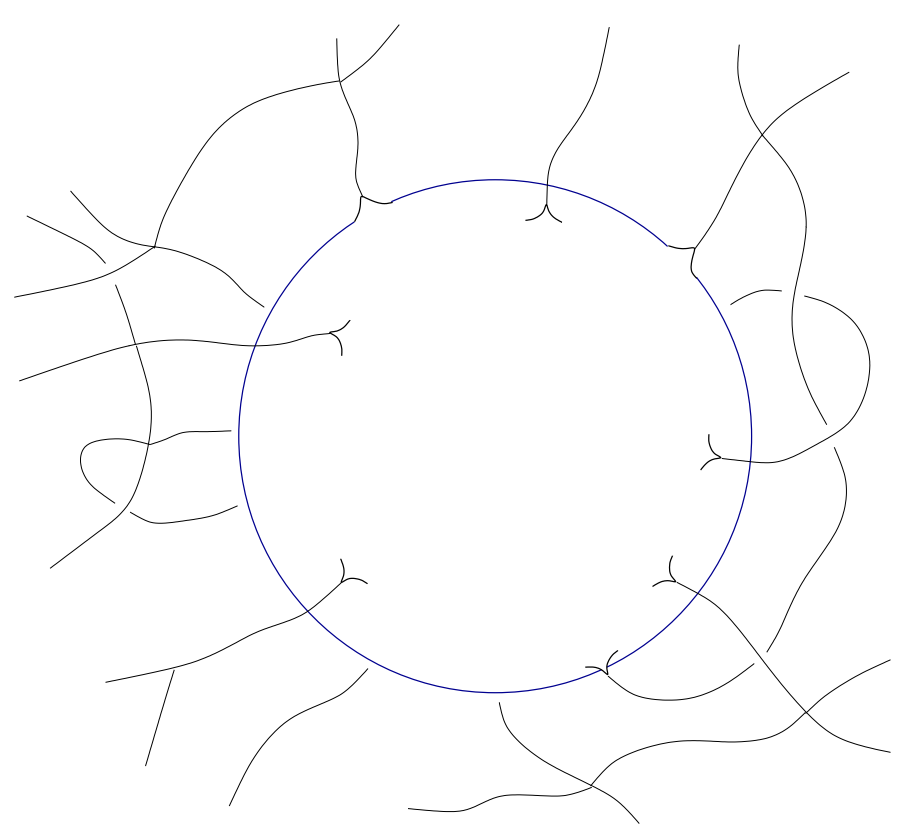

Figure 12: Quantum horizon. Polymer excitations in the bulk puncture the horizon, endowing it with quantized area. Intrinsically, the horizon is flat except at punctures where it acquires a quantized deficit angle. These angles add up to endow the horizon with a 2-sphere topology.

An important subtlety arises because the operators corresponding to the two sides of Equa- 
tion (89) act on different Hilbert spaces: While $\hat{F}$ is defined on $\mathcal{H}_{S}, \hat{\Sigma} \cdot r$ is defined on $\mathcal{H}_{V}$. Therefore, the quantum horizon boundary condition introduces a precise intertwining between the bulk and the surface states: Only those states $\Psi=\sum_{i} \Psi_{V}^{i} \otimes \Psi_{S}^{i}$ in $\mathcal{H}$ which satisfy

$$
(1 \otimes \hat{F}) \Psi=\left(-\frac{2 \pi \gamma}{a_{0}} \hat{\sum} \cdot r \otimes 1\right) \Psi
$$

can describe quantum geometries with WIH as inner boundaries. This is a stringent restriction: Since the operator on the left side acts only on surface states and the one on the right side acts only on bulk states, the equation can have solutions only if the two operators have the same eigenvalues (in which case we can take $\Psi$ to be the tensor product of the two eigenstates). Thus, for solutions to Equation (93) to exist, there has to be a very delicate matching between eigenvalues of the triad operators $\hat{\Sigma} \cdot r$ calculated from bulk quantum geometry, and eigenvalues of $\hat{F}$, calculated from Chern-Simons theory. The precise numerical coefficients in the surface calculation depend on the numerical factor in front of the surface term in the symplectic structure, which is itself determined by our type I WIH boundary conditions. Thus, the existence of a coherent quantum theory of WIHs requires that the three cornerstones - classical isolated horizon framework, quantum mechanics of bulk geometry, and quantum Chern-Simons theory - be united harmoniously. Not only should the three frameworks be mutually compatible at a conceptual level, but certain numerical coefficients, calculated independently within each framework, have to match delicately. Remarkably, these delicate constraints are met, whence the quantum boundary conditions do admit a sufficient number of solutions.

We will conclude by summarizing the nature of geometry of the quantum horizon that results. Given any state satisfying Equation (93), the curvature $F$ of $W$ vanishes everywhere except at the points at which the polymer excitations in the bulk puncture $S$. The holonomy around each puncture is non-trivial. Consequently, the intrinsic geometry of the quantum horizon is flat except at the punctures. At each puncture, there is a deficit angle, whose value is determined by the holonomy of $W$ around that puncture. Each deficit angle is quantized and these angles add up to $4 \pi$ as in a discretized model of a 2 -sphere geometry. Thus, the quantum geometry of a WIH is quite different from its smooth classical geometry.

\section{$7.3 \quad$ Entropy}

Let us now summarize the ideas behind counting of surface microstates that leads to the expression of entropy. To incorporate dynamics in this canonical approach, we have to first construct physical states by imposing quantum Einstein equations (i.e., quantum constraints). While the procedure is technically quite involved, the result is simple to state: What matters is only the number of punctures and not their locations. To calculate entropy, then, one constructs a micro-canonical ensemble as follows. Fix the number $n$ of punctures and allow only those (non-zero) spin-labels $j_{I}$ and charge labels $q_{I}^{\alpha}$ on the polymer excitations which endow the horizon with a total area in an interval $\left(a_{0}-\epsilon, a_{0}+\epsilon\right)$ and charges in an interval $\left(Q_{0}^{\alpha}-\epsilon^{\alpha}, Q_{0}^{\alpha}+\epsilon^{\alpha}\right)$. (Here $I=1,2, \ldots, n$ and $\epsilon$ and $\epsilon^{\alpha}$ are suitably small. Their precise values will not affect the leading contribution to entropy.) We denote by $\mathcal{H}_{n}^{\mathrm{BH}}$ the sub-space of $\mathcal{H}=\mathcal{H}_{V} \otimes \mathcal{H}_{S}$ in which the volume states $\Psi_{V}$ are chosen with the above restrictions on $j_{I}$ and $q_{I}^{\alpha}$, and the total state $\Psi$ satisfies the quantum horizon boundary condition as well as quantum Einstein equations. Then the desired micro-canonical ensemble consists of states in $\mathcal{H}^{\mathrm{BH}}=\oplus_{n} \mathcal{H}_{n}^{\mathrm{BH}}$. Note that, because there is no contribution to the symplectic structure from matter terms, surface states in $\mathcal{H}^{\mathrm{BH}}$ refer only to the gravitational sector.

The next step is to calculate the entropy of this quantum, micro-canonical ensemble. Note first that what matters are only the surface states. For, the 'bulk-part' describes, e.g., states of gravitational radiation and matter fields far away from $\Delta$ and are irrelevant for the entropy $S_{\Delta}$ of the WIH. Heuristically, the idea then is to 'trace over' the bulk states, construct a density matrix

Living Reviews in Relativity

http://www . livingreviews . org//rr-2004-10 
$\rho_{\mathrm{BH}}$ describing a maximum-entropy mixture of surface states and calculate $\operatorname{tr} \rho_{\mathrm{BH}} \ln \rho_{\mathrm{BH}}$. As is usual in entropy calculations, this translates to the evaluation of the dimension $\mathcal{N}$ of a well-defined sub-space $\mathcal{H}_{S}^{\mathrm{BH}}$ of the surface Hilbert space, namely the linear span of those surface states which occur in $\mathcal{H}^{\mathrm{BH}}$. Entropy $S_{\Delta}$ is given by $\ln \mathcal{N}$.

A detailed calculation $[84,149]$ leads to the following expression of entropy:

$$
S_{\Delta}=\frac{\gamma_{0}}{\gamma} \frac{a_{0}}{4 \ell_{\mathrm{Pl}}^{2}}-\frac{1}{2} \ln \frac{a_{0}}{\ell_{\mathrm{Pl}}^{2}}+\mathcal{O}\left(\ln \frac{a_{0}}{\ell_{\mathrm{Pl}}^{2}}\right), \quad \text { with } \gamma_{0}=\approx 0.2375,
$$

where $\mathcal{O}\left(\ln \left(a_{0} / \ell_{\mathrm{Pl}}^{2}\right)\right)$ is a term which, when divided by $a_{0} / \ell_{\mathrm{Pl}}^{2}$, tends to zero as $a_{0} / \ell_{\mathrm{p}}^{2}$ tends to infinity. Thus, for large black holes, the leading term in the expression of entropy is proportional to area. This is a non-trivial result. For example if, as in the early treatments [170, 36, 163, 164, 134, 135] one ignores the horizon boundary conditions and the resulting Chern-Simons term in the symplectic structure, one would find that $S_{\Delta}$ is proportional to $\sqrt{a_{0}}$. However, the theory does not have a unique prediction because the numerical coefficient depends on the value of the Barbero-Immirzi parameter $\gamma$. The appearance of $\gamma$ can be traced back directly to the fact that, in the $\gamma$-sector of the theory, the area eigenvalues are proportional to $\gamma$.

One adopts a 'phenomenological' viewpoint to fix this ambiguity. In the infinite dimensional space of geometries admitting $\Delta$ as their inner boundary, one can fix one space-time, say the Schwarzschild space-time with mass $M_{0} \gg M_{\mathrm{Pl}}$, (or, the de Sitter space-time with the cosmological constant $\Lambda_{0} \ll 1 / \ell_{\mathrm{Pl}}^{2}$, or,$\left.\ldots\right)$. For agreement with semi-classical considerations in these cases, the leading contribution to entropy should be given by the Hawking-Bekenstein formula (87). This can happen only in the sector $\gamma=\gamma_{0}$. The quantum theory is now completely determined through this single constraint. We can go ahead and calculate the entropy of any other type I WIH in this theory. The result is again $S_{\Delta}=S_{\mathrm{BH}}$. Furthermore, in this $\gamma$-sector, the statistical mechanical temperature of any type I WIH is given by Hawking's semi-classical value $\kappa \hbar /(2 \pi)[29,135]$. Thus, we can do one thought experiment - observe the temperature of one large black hole from far away - to eliminate the Barbero-Immirzi ambiguity and fix the theory. This theory then predicts the correct entropy and temperature for all WIHs with $a_{0} \gg \ell^{2}$, irrespective of other parameters such as the values of the electric or dilatonic charges or the cosmological constant. An added bonus comes from the fact that the isolated horizon framework naturally incorporates not only black hole horizons but also the cosmological ones for which thermodynamical considerations are also known to apply [99]. The quantum entropy calculation is able to handle both these horizons in a single stroke, again for the same value $\gamma=\gamma_{0}$ of the Barbero-Immirzi parameter. In this sense, the prediction is robust.

Finally, these results have been subjected to further robustness tests. The first comes from nonminimal couplings. Recall from Section 6 that in presence of a scalar field which is non-minimally coupled to gravity, the first law is modified $[123,183,184]$. The modification suggests that the Hawking-Bekenstein formula $S_{\mathrm{BH}}=a_{0} /\left(4 \ell_{\mathrm{Pl}}^{2}\right)$ is no longer valid. If the non-minimal coupling is dictated by the action

$$
\widetilde{S}\left[g_{a b}, \phi\right]=\int\left(\frac{1}{16 \pi G} f(\phi) R-\frac{1}{2} g^{a b} \nabla_{a} \phi \nabla_{b} \phi-V(\phi)\right) \sqrt{-g} d^{4} x,
$$

where $R$ is the scalar curvature of the metric $g_{a b}$ and $V$ is a potential for the scalar field, then the entropy should be given by [22]

$$
S_{\Delta}=\frac{1}{4 G \hbar} \oint f(\phi) d^{2} V
$$

An immediate question arises: Can the calculation be extended to this qualitatively new situation? At first, this seems very difficult within an approach based on quantum geometry, such as the one described above, because the relation is non-geometric. However, the answer was shown to be in 
the affirmative [20] for type I horizons. It turns out that the metric $\bar{q}_{a b}$ on $M$ is no longer coded in the gravitational momentum $P_{i}^{a}$ alone but depends also on the scalar field. This changes the surface term $\boldsymbol{\Omega}_{S}$ in the symplectic structure (90) as well as the horizon boundary condition (89) just in the right way for the analysis to go through. Furthermore, state counting now leads to the desired expression (96) precisely for the same value $\gamma=\gamma_{0}$ of the Barbero-Immirzi parameter [20].

Next, one can consider type II horizons which can be distorted and rotating. In this case, all the (gravitational, electro-magnetic, and scalar field) multipoles are required as macroscopic parameters to fix the system of interest. Therefore, now the appropriate ensemble is determined by fixing all these multipoles to lie in a small range around given values. This ensemble can be constructed by first introducing multipole moment operators and then restricting the quantum states to lie in the subspace of the Hilbert space spanned by their eigenvectors with eigenvalues in the given intervals. Again recent work shows that the state counting yields the HawkingBekenstein formula (87) for minimally coupled matter and its modification (96) for non-minimally coupled scalar field, for the same value $\gamma=\gamma_{0}$ of the Barbero-Immirzi parameter [8, 25].

To summarize, the isolated horizon framework serves as a natural point of departure for a statistical mechanical calculation of black hole entropy based on quantum geometry. How does this detailed analysis compare with the 'It from Bit' scenario [187] with which we began? First, the quantum horizon boundary conditions play a key role in the construction of a consistent quantum theory of the horizon geometry. Thus, unlike in the 'It from Bit' scenario, the calculation pertains only to those 2-spheres $S$ which are cross-sections of a WIH. One can indeed divide the horizon into elementary cells as envisaged by Wheeler: Each cell contains a single puncture. However, the area of these cells is not fixed but is dictated by the $j$-label at the puncture. Furthermore, there are not just 2 but rather $2 j+1$ states associated with each cell. Thus, the complete theory is much more subtle than that envisaged in the 'It from Bit' scenario.

Living Reviews in Relativity

http://www . livingreviews . org//rr-2004-10 


\section{Outlook}

In the last six sections, we summarized the isolated and dynamical horizon frameworks and their applications. These provide a quasi-local and more physical paradigm for describing black holes both in the equilibrium and dynamical regimes. One of the most pleasing aspects of the paradigm is that it provides a unified approach to a variety of problems involving black holes, ranging from entropy calculations in quantum gravity, to analytical issues related to numerical simulations, to properties of hairy black holes, to laws of black hole mechanics. More importantly, as summarized in Section 1, these frameworks enable one to significantly extend the known results based on Killing and event horizons, and provide brand new laws in the dynamical regime.

In this section, we will discuss some of the open issues whose resolution would lead to significant progress in various areas.

\section{Isolated horizons}

This is the best understood part of the new paradigm. Nonetheless, several important issues still remain. We will illustrate these with a few examples:

\section{Black hole mechanics}

Throughout, we assumed that the space-time metric is $C^{k}$ (with $k \geq 3$ ) and the topology of $\Delta$ is $S^{2} \times R$. These assumptions rule out, by fiat, the presence of a NUT charge. To incorporate a non-zero NUT charge in black hole mechanics, one must either allow $\Delta$ to be topologically $S^{3}$, or allow for 'wire singularities' in the rotation 1-form $\omega_{a}$ on $\Delta$. The zeroth law goes through in these more general situations. What about the first law? Arguments based on Euclidean methods [114, 146, 147] show that entropy is no longer given by the horizon area but there is also contribution due to 'Misner strings'. However, to our knowledge, a systematic derivation in the Lorentzian regime is not yet available. Such a derivation would provide a better understanding of the physical origin of the extra terms. The covariant phase space methods used in the isolated horizon framework should be applicable in this case.

\section{Application to numerical relativity}

We saw in Section 5.4 that, in the IH framework, one can introduce an approximate analog of future null infinity $\mathcal{I}^{+}$and invariant coordinate systems and tetrads in its neighborhood. With this structure, it is feasible to extract waveforms and energy fluxes in a reliable manner within the standard $3+1$ Cauchy evolution of numerical relativity, without having to do a Cauchy characteristic matching or use conformal field equations. The challenge here is to develop, on the approximate $\mathcal{I}^{+}$, the analog of the basics of the Bondi framework [54, 33, 185] at null infinity.

Colored black holes

As discussed in Section 6, black hole uniqueness theorems of the Einstein-Maxwell theory fail once non-Abelian fields are included. For example, there are black hole solutions to the Einstein-Yang-Mills equations with non-trivial Yang-Mills fields whose only nonzero charge at infinity is the ADM mass. Thus, from the perspective of infinity, they are indistinguishable from the Schwarzschild solution. However, their horizon properties are quite different from those of the Schwarzschild horizon. This example suggests that perhaps the uniqueness theorems fail because of the insistence on evaluating charges at infinity. Corichi, Nucamendi, and Sudarsky [75] have conjectured that the uniqueness theorems could be restored if they are formulated in terms of all the relevant horizon charges. This is a fascinating idea and it has been pursued numerically. However, care is needed because the list of all relevant charges may not be obvious a priori. For example, in the case of static but not necessarily spherical Yang-Mills black holes, the conjecture seemed to fail [132] until one realized that, in addition to the standard Yang-Mills 
charges at the horizon, one must also include a topological, 'winding charge' in the list [9]. Once this charge is included, uniqueness is restored not only in the static sector of the Einstein-Yang-Mills theory, but also when Higgs fields [109] and dilatons [131] are included. The existence of these semi-numerical proofs suggests that it should be possible to establish uniqueness completely analytically. A second set of problems involves the surprising relations, e.g., between the soliton masses and horizon properties of colored black holes, obtained using isolated horizons. Extensions of these results to situations with non-zero angular momentum should be possible and may well provide yet new insights.

Quantum black holes

In the approach based on isolated horizons, the microscopic degrees of freedom responsible for the statistical mechanical entropy of black holes are directly related to the quantum geometry of horizons. Therefore, their relation to the curved space-time geometry is clearer than in, say, the string theory calculations based on D-branes. Therefore, one can now attempt to calculate the Hawking radiation directly in the physical spacetime as a process in which quanta of area are converted to quanta of matter. However, such a calculation is yet to be undertaken. A direct approach requires quantum field theory (of matter fields) on a quantum geometry state which is approximated by the classical black hole space-time. Elements of this theory are now in place. The concrete open problem is the calculation of the absorption cross-section for quantum matter fields propagating on this 'background state'. If this can be shown to equal the classical absorption cross-section to the leading order, it would follow [29] that the spectrum of the outgoing particles would be thermal at the Hawking temperature. Another, perhaps more fruitful, avenue is to introduce an effective model whose Hamiltonian captures the process by which quanta of horizon area are converted to quanta of matter. Both these approaches are geared to large black holes which can be regarded as being in equilibrium for the process under consideration, i.e., when $a_{\Delta} \gg \ell_{\mathrm{Pl}}^{2}$. However, this approximation would fail in the Planck regime whence the approaches can not address issues related to 'information loss'. Using ideas first developed in the context of quantum cosmology, effects of the quantum nature of geometry on the black hole singularity have recently been analyzed [17]. As in the earlier analysis of the big-bang singularity, it is found that the black hole singularity is resolved, but the classical space-time dissolves in the Planck regime. Therefore, the familiar Penrose diagrams are no longer faithful representations of the physical situation. Suppose that, evolving through what was singularity in the classical theory, the quantum state becomes semi-classical again on the 'other side'. Then, the indications are that information would not be lost: It would be recovered on full $\mathcal{I}^{+}$, although observers restricted to lie in the part of space-time which is completely determined by the data on $\mathcal{I}^{-}$would see an approximate Hawking radiation. If on the other hand the evolved state on the 'other side' never becomes semi-classical, information would not be recovered on the available $\mathcal{I}^{+}$. An outstanding open issue is which of this possibility is actually realized.

\section{Dynamical horizons}

The DH framework is less developed and the number of open issues is correspondingly higher. At least for the classical applications of the framework, these problems are more important.

Free data and multipoles

Since $H$ is space-like, to find the fields $\left(q_{a b}, K_{a b}\right)$ which constitute the DH geometry, one has to solve just the initial value equations, subject to the condition that $H$ admits a foliation by marginally trapped surfaces. A general solution of this problem would provide the 'free data' on DHs. In the case when the marginally trapped surfaces are

Living Reviews in Relativity

http://www. livingreviews . org//rr-2004-10 
round spheres, this problem has been analyzed by Bartnik and Isenberg [37]. As noted in Section 2.2, in this case there are no DHs in absence of matter sources. In presence of matter, one can freely specify the trace $K$ of the extrinsic curvature and the (radial component of the) momentum density $T_{a b} \hat{\tau}^{a} \hat{r}^{b}$, and determine the geometry by solving a non-linear ordinary differential equation whose solutions always exist locally. It would be interesting to analyze the necessary and sufficient conditions on the free data which guarantee that global solutions exist and the DH approaches the Schwarzschild horizon asymptotically. From the point of view of numerical relativity, a more pressing challenge is to solve the constraint equations in the vacuum case, assuming only that the marginally trapped surfaces are axi-symmetric. Using the free data, as in the case of isolated horizons [24], one could define multipoles. Since $\Psi_{2}$ is again defined unambiguously, a natural starting point is to use it as the key geometrical object as in the IH case. However, just as the Bondi mass aspect acquires shear terms in presence of gravitational radiation [54], it is likely that, in the transition from isolated to dynamical horizons, $\Psi_{2}$ would have to be supplemented with terms involving, e.g., $\sigma_{a b}$ (and perhaps also $\zeta^{a}$ ). For instance, by adding a suitable combination of such terms, one may be able to relate the rate of change of the mass quadrupole moment with the flux of energy across $H$.

Geometric analysis

The dynamical horizon framework provides new inputs for the proof of Penrose inequalities which, when applied to time symmetric data (i.e., when the extrinsic curvature vanishes), say that the total (ADM) mass of space-time must be greater than half the radius of the apparent horizon on any Cauchy slice. This conjecture was recently proved by Bray [59], and by Huisken and Ilmamen [122]. Recently, for the non-time symmetric case, Ben-Dov has constructed an example where the apparent horizon does not satisfy this inequality [47]. This is not a contradiction with the original Penrose inequality which referred to the area of cross-sections of the event horizon, however it does show that extending the results beyond time-symmetry would be quite non-trivial. The 'flows' which led to the area law in Section 3 and balance equations in Section 4.2.2 may be potentially useful for this purpose. This approach could lead to an inequality relating the area of certain marginally trapped surfaces (the ones connected to future time like infinity via a dynamical horizon) to the future limit of the Bondi mass. The framework also suggests a program which could shed much light on what John Wheeler called 'the issue of the final state': what are the final equilibrium states of a dynamical black hole and how, in detail, is this equilibrium reached? From a mathematical perspective an important step in addressing this issue is to analyze the non-linear stability of Kerr black holes. Consider, then, a neighborhood of the initial data of the Kerr solution in an appropriate Sobolev space. One would expect the space-time resulting from evolution of this data to admit a dynamical horizon which, in the distant future, tends to an isolated horizon with geometry that is isomorphic to that of a Kerr horizon. Can one establish that this is what happens? Can one estimate the 'rate' with which the Kerr geometry is approached in the asymptotic future? One avenue is to first establish that the solution admits a Kerr-Schild type foliation, each leaf of which admits an apparent horizon, then show that the world tube of these apparent horizons is a $\mathrm{DH}$, and finally study the decay rates of fields along this DH.

Angular momentum

As we saw in Section 5, most of the current work on IHs and DHs assumes the presence of an axial symmetry $\phi^{a}$ on the horizon. A natural question arises: Can one weaken this requirement to incorporate situations in which there is only an approximate - rather than an exact - symmetry of the horizon geometry? The answer is in the affirmative in the following sense. Recall first that the Newman-Penrose component $\Psi_{2}$ is gauge 
invariant on IHs and DHs. Let $F=\left|\Psi_{2}\right|^{2}$. (While any geometric field could be used here, $F$ is the most natural candidate because, for IHs, $\Psi_{2}$ encodes the horizon geometry.) If the horizon geometry admits a symmetry, the orbits of the symmetry field $\phi^{a}$ are the level surfaces of $F$. More generally, let us suppose that the level surfaces of $F$ provide a foliation of each good cut $S$ (minus two points) of the horizon. Then, using the procedure of section 2.1 of [24], one can introduce on $S$ a vector field $\phi_{0}^{a}$, tangential to the foliation, which has the property that it agrees with the symmetry vector field $\phi^{a}$ whenever the horizon geometry admits a symmetry. The procedure fails if the metric on $S$ is at of a round sphere but should work generically. One can then use $\phi_{0}^{a}$ to define angular momentum. On IHs this angular momentum is conserved; on DHs it satisfies the balance law of Section 4.2.2; and in terms of the initial data, angular momentum $J^{\left(\phi_{0}\right)}$ has the familiar form (59). IS this proposal viable for numerical simulations? In cases ready analyzed, it would be interesting to construct $\phi_{0}^{a} \mathrm{~d}$ compare it with the symmetry vector field obtained via Killing transport. A better test would be provided by non-axisymmetric Brill waves. A second issue associated with angular momentum is ether the Kerr inequality $J \leq G M^{2}$ can be violated in the early stages of black hole formation or merger, particularly in a non-axisymmetric context. Equations that must hold on DHs provide no obvious obstruction [31]. Note that such an occurrence is not incompatible with the DH finally settling down to a Kerr horizon. For, there is likely to be radiation trapped between the DH and the 'peak' of the effective gravitational potential that could fall into the $\mathrm{DH}$ as time elapses, reducing its angular momentum and increasing its mass. The issue of whether a black hole can violate the Kerr inequality when it is first formed is of considerable interest to astrophysics [89]. Again, numerical simulations involving, say, Brill waves would shed considerable light on this possibility.

\section{Black hole thermodynamics}

The fact that an integral version of the first law is valid even for non-equilibrium processes, during which the horizon makes a transition from a given state to one which is far removed, has interesting thermodynamic ramifications. In non-equilibrium thermodynamical processes, in general the system does not have time to come to equilibrium, whence there is no canonical notion of its temperature. Therefore, while one can still interpret the difference $E_{2}-E_{1}-$ (work) as the heat $\Delta Q$ absorbed by the system, in general there is no longer a clean split $\Delta Q=T \Delta S$ of this term into a temperature part and a change in entropy part. If the process is such that the system remains close to equilibrium throughout the process, i.e., can be thought of as making continuous transitions between a series of equilibrium states, then the difference can be expressed as $\int T d S$, where the temperature $T$ varies slowly during the transition. The situation on dynamical horizons is analogous. It is only when the horizon geometry is changing slowly that the effective surface gravity $\bar{\kappa}$ of Section 4.2 .2 would be a good measure of temperature, and the horizon area a good measure of entropy (see Section 5.3 of [31]). These restricted situations are nonetheless very interesting. Can the black hole entropy derivations based on counting of micro-states, such as those of [10], be extended to such DHs? In the case of event horizons one would not expect such a procedure to be meaningful because, as we saw in Equation 2.2.2, an event horizon can be formed and grow in a flat space region in anticipation of a future gravitational collapse. It is difficult to imagine how a quasi-local counting of micro-states can account for this phenomenon.

Perhaps the most surprising aspect of the current status of the theory of black holes is that so little is known about their properties in the fully dynamical and non-linear regime of general relativity. Indeed, we do not even have a fully satisfactory definition of a dynamical black hole. Traditionally, one uses event horizons. But as we discussed in detail, they have several undesirable

Living Reviews in Relativity

http://www . livingreviews . org//rr-2004-10 
features. First, they are defined only in space-times where one can unambiguously identify infinity. Even in these restricted contexts, event horizons are teleological, can form in a flat region of spacetime and grow even though there is no flux of energy of any kind across them. When astronomers tell us that there is a black hole in the center of Milky Way, they are certainly not referring to event horizons.

Numerical simulations [48, 102] suggest that the outermost marginally trapped world-tubes become dynamical horizons soon after they are formed. As we saw, dynamical horizons have a number of attractive properties that overcome the limitations of event horizons: they are defined quasi-locally, can not be formed in flat space-time, and their growth is dictated by balance laws with direct physical interpretation. Physically, then, dynamical horizons satisfying the additional physical condition $\mathcal{L}_{n} \Theta_{(\ell)}<0$ (i.e., SFOTHs) appear to be good candidates to represent the surface of a black hole. But so far, our understanding of their uniqueness is rather limited. If a canonical dynamical horizon could be singled out by imposing physically reasonable conditions, one could use it as the physical representation of an evolving black hole.

A plausibility argument for the existence of a canonical dynamical horizon was given by Hayward. Note first that on physical grounds it seems natural to associate a black hole with a connected, trapped region $\mathcal{T}$ in space-time (see Section 2.2.1). Hayward [117] sketched a proof that, under seemingly natural but technically strong conditions, the dynamical portion of its boundary, $\partial \mathcal{T}$, would be a dynamical horizon $H$. This $H$ could serve as the canonical representation of the surface of an evolving black hole. However, it is not clear whether Hayward's assumptions are not too strong. To illustrate the concern, let us consider a single black hole. Then, Hayward's argument implies that there are no trapped surfaces outside $H$. On the other hand there has been a general expectation in the community that, given any point in the interior of the event horizon, there passes a (marginally) trapped surface through it (see, e.g., [86]). This would imply that the boundary of the trapped region is the event horizon which, being null, can not qualify as a dynamical horizon. However, to our knowledge, this result has not been firmly established. But it is clear that this expectation contradicts the conclusion based on Hayward's arguments. Which of these two expectations is correct? It is surprising that such a basic issue is still unresolved. The primary reason is that very little is known about trapped and marginally trapped surfaces which fail to be spherical symmetric. Because of this, we do not know the boundary of the trapped region even in the Vaidya solution.

If it should turn out that the second expectation is correct, one would conclude that Hayward's assumptions on the properties of the boundary of the trapped region are not met in physically interesting situations. However, this would not rule out the possibility of singling out a canonical dynamical horizon through some other conditions (as, e.g., in the Vaidya solution). But since this dynamical horizon would not be the boundary of the trapped region, one would be led to conclude that in the dynamical and fully non-linear regime, one has to give up the idea that there is a single 3-manifold that can be interpreted as the black hole surface without further qualifications. For certain questions and in certain situations, the dynamical horizon may be the appropriate concept, while for other questions and in different situations, the boundary of the trapped region (which may be the event horizon) may be more appropriate. 


\section{Acknowledgements}

We are grateful to John Baez, Chris Beetle, Alejandro Corichi, Sergio Dain, Olaf Dreyer, Jonathan Engle, Stephen Fairhurst, Greg Galloway, Sean Hayward, Jose Luis Jaramillo, Jerzy Lewandowski, Kirill Krasnov, Tomasz Pawlowski, Erik Schnetter, Deirdre Shoemaker, Daniel Sudarsky, Chris van den Broeck, and Jacek Wisniewski for collaboration on several of the results summarized in this review. We have also benefited from stimulating discussions with numerous colleagues, especially among them Marcus Ansorg, Ivan Booth, Bobby Beig, Piotr Bizoń, Piotr Chruściel, Peter Diener, Sam Finn, Éanna Flanagan, Jim Hartle, Ian Hawke, Gary Horowitz, Gerhard Huisken, Jim Isenberg, Pablo Laguna, Luis Lehner, Richard Matzner, Jorge Pullin, Oscar Reula, Bernd Schmidt, Walter Simon, Ken Smith, Josh Willis, and Jeff Winicour. This work was supported in part by the National Science Foundation grants PHY-0090091, the National Science Foundation Cooperative Agreement PHY-0114375, the Albert-Einstein-Institut, the Erwin-Schrödinger-Institut, the Kavli Institute of Theoretical Physics, the Alexander von Humboldt Foundation, and the Eberly research funds of Penn State.

Living Reviews in Relativity

http: //www . livingreviews . org/Irr-2004-10 


\section{References}

[1] Abrahams, A.M., Rezzolla, L., Rupright, M.E., Anderson, A., Anninos, P., Baumgarte, T.W., Bishop, N.T., Brandt, S.R., Browne, J.C., Camarda, K., Choptuik, M.W., Cook, G.B., Evans, C.R., Finn, L.S., Fox, G., Gómez, R., Haupt, T., Huq, M.F., Kidder, L.E., Klasky, S., Laguna, P., Landry, W., Lehner, L., Lenaghan, J., Marsa, R.L., Massó, J., Matzner, R.A., Mitra, S., Papadopoulos, P., Parashar, M., Saied, F., Saylor, P.E., Scheel, M.A., Seidel, E., Shapiro, S.L., Shoemaker, D.M., Smarr, L.L., Szilágyi, B., Teukolsky, S.A., van Putten, M.H.P.M., Walker, P., Winicour, J., and York Jr, J.W. (The Binary Black Hole Grand Challenge Alliance), "Gravitational wave extraction and outer boundary conditions by perturbative matching", Phys. Rev. Lett., 80, 1812-1815, (1998). For a related online version see: A.M. Abrahams, et al., (September, 1997), [Online Los Alamos Archive Preprint]: cited on 22 November 2004,

http://arXiv.org/abs/gr-qc/9709082. 1

[2] Alcubierre, M., Benger, W., Brügmann, B., Lanfermann, G., Nerger, L., Seidel, E., and Takahashi, R., "3D Grazing Collision of Two Black Holes", Phys. Rev. Lett., 87, 271103-14, (2001). For a related online version see: M. Alcubierre, et al., (December, 2000), [Online Los Alamos Archive Preprint]: cited on 22 November 2004, http://arXiv.org/abs/gr-qc/0012079. 1

[3] Alcubierre, M., Brügmann, B., Pollney, D., Seidel, E., and Takahashi, R., "Black hole excision for dynamic black holes", Phys. Rev. D, 64, 061501-1-5, (2001). For a related online version see: M. Alcubierre, et al., (April, 2001), [Online Los Alamos Archive Preprint]: cited on 22 November 2004,

http://arXiv.org/abs/gr-qc/0104020. 5, 5.2.1

[4] Andrade, Z., Beetle, C., Blinov, A., Bromley, B., Burko, L.M., Cranor, M., Owen, R., and Price, R.H., "Periodic standing-wave approximation: Overview and three-dimensional scalar models", Phys. Rev. D, 70, 064001-1-14, (2003). 5.2 .1

[5] Anninos, P., Bernstein, D., Brandt, S., Hobill, D., Seidel, E., and Smarr, L.L., "Dynamics of Black Hole Apparent Horizons", Phys. Rev. D, 50, 3801-3819, (1994). 2.1.1

[6] Anninos, P., Camarda, K., Libson, J., Massó, J., Seidel, E., and Suen, W.-M., "Finding apparent horizons in dynamic 3D numerical spacetimes", Phys. Rev. D, 58, 24003-1-12, (1998). For a related online version see: P. Anninos, et al., (September, 1996), [Online Los Alamos Archive Preprint]: cited on 22 November 2004,

http://arXiv.org/abs/gr-qc/9609059. 5

[7] Arnowitt, R., Deser, S., and Misner, C.W., "The dynamics of general relativity", in Witten, L., ed., Gravitation: An introduction to current research, 227-265, (Wiley, New York, U.S.A., 1962). $4.1 .2,5$

[8] Ashtekar, A., "Black Hole Entropy: Inclusion of Distortion and Angular Momentum", (2003), [Online Presentation]: cited on 22 November 2004,

http://www.phys.psu.edu/events/index.html?event_id=517. $\quad 7.2,7.3$

[9] Ashtekar, A., Personal communication to Corichi, A., Kleihaus, B., and Kunz, J., (2002). 8

[10] Ashtekar, A., Baez, J., Corichi, A., and Krasnov, K., "Quantum geometry and black hole entropy", Phys. Rev. Lett., 80, 904-907, (1998). For a related online version see: A. Ashtekar, et al., (1997), [Online Los Alamos Archive Preprint]: cited on 22 November 2004, http://arXiv.org/abs/gr-qc/9710007. 1, 7.2,8 
[11] Ashtekar, A., Baez, J., and Krasnov, K., "Quantum Geometry of Isolated Horizons and Black Hole Entropy", Adv. Theor. Math. Phys., 4, 1-94, (2000). For a related online version see: A. Ashtekar, et al., (2000), [Online Los Alamos Archive Preprint]: cited on 22 November 2004 http://arXiv.org/abs/gr-qc/0005126. 1, 7.2

[12] Ashtekar, A., Beetle, C., Dreyer, O., Fairhurst, S., Krishnan, B., Lewandowski, J., and Wisniewski, J., "Generic isolated horizons and their applications", Phys. Rev. Lett., 85, 3564-3567, (2000). For a related online version see: A. Ashtekar, et al., (2000), [Online Los Alamos Archive Preprint]: cited on 22 November 2004,

http://arXiv.org/abs/gr-qc/0006006. 1, 5.4

[13] Ashtekar, A., Beetle, C., and Fairhurst, S., "Isolated Horizons: A Generalization of Black Hole Mechanics", Class. Quantum Grav., 16, L1-L7, (1999). For a related online version see: A. Ashtekar, et al., (1998), [Online Los Alamos Archive Preprint]: cited on 22 November 2004 , http://arXiv.org/abs/gr-qc/9812065. 2.1

[14] Ashtekar, A., Beetle, C., and Fairhurst, S., "Mechanics of isolated horizons", Class. Quantum Grav., 17, 253-298, (2000). For a related online version see: A. Ashtekar, et al., (1999), [Online Los Alamos Archive Preprint]: cited on 22 November 2004, http://arXiv.org/abs/gr-qc/9907068. 2.1, 4.1.3, 4.2.2

[15] Ashtekar, A., Beetle, C., and Lewandowski, J., "Mechanics of rotating isolated horizons", Phys. Rev. D, 64, 044016-1-17, (2001). For a related online version see: A. Ashtekar, et al., (2001), [Online Los Alamos Archive Preprint]: cited on 22 November 2004, http://arXiv.org/abs/gr-qc/0103026. 1, 2.1.3, 4.1, 4.1.2, 4.1.2, 4.1.3, 4.1.3, 4.3

[16] Ashtekar, A., Beetle, C., and Lewandowski, J., "Geometry of generic isolated horizons", Class. Quantum Grav., 19, 1195-1225, (2002). For a related online version see: A. Ashtekar, et al., (2001), [Online Los Alamos Archive Preprint]: cited on 22 November 2004, http://arXiv.org/abs/gr-qc/0111067. 2.1, 2.1.1, 2.1.1, 2.1.3, 2.1.3, 4.1.2, 6

[17] Ashtekar, A., and Bojowald, M., in preparation. 8

[18] Ashtekar, A., Bombelli, L., and Reula, O.A., "Covariant phase space of asymptotically flat gravitational fields", in Francaviglia, M., and Holm, D., eds., Mechanics, Analysis and Geometry: 200 Years after Lagrange, 417-450, North-Holland Delta Series, (North Holland, Amsterdam, Netherlands; New York, U.S.A., 1990). 4.1.2, 5

[19] Ashtekar, A., and Corichi, A., "Laws governing isolated horizons: Inclusion of dilaton coupling", Class. Quantum Grav., 17, 1317-1332, (2000). For a related online version see: A. Ashtekar, et al., (October, 1999), [Online Los Alamos Archive Preprint]: cited on 22 November 2004, http://arXiv.org/abs/gr-qc/9910068. 4.1, 6

[20] Ashtekar, A., and Corichi, A., "Non-minimal couplings, quantum geometry and black hole entropy", Class. Quantum Grav., 20, 4473-4484, (2003). 1, 7.2, 7.3

[21] Ashtekar, A., Corichi, A., and Sudarsky, D., "Hairy black holes, horizon mass and solitons", Class. Quantum Grav., 18, 919-940, (2001). For a related online version see: A. Ashtekar, et al., (November, 2000), [Online Los Alamos Archive Preprint]: cited on 22 November 2004, http://arXiv.org/abs/gr-qc/0011081. 1, 6, 6.2.2, 6.2.2, 6.2.3, 6.2.3, 6.2.3

Living Reviews in Relativity

http://www. livingreviews . org//rr-2004-10 
[22] Ashtekar, A., Corichi, A., and Sudarsky, D., "Non-Minimally Coupled Scalar Fields and Isolated Horizons", Class. Quantum Grav., 20, 3513-3425, (2003). 6, 7.3

[23] Ashtekar, A., Dreyer, O., and Wisniewski, J., "Isolated Horizons in 2+1 Gravity", Adv. Theor. Math. Phys., 6, 507-555, (2002). For a related online version see: A. Ashtekar, et al., (June, 2002), [Online Los Alamos Archive Preprint]: cited on 22 November 2004, http://arXiv.org/abs/gr-qc/0206024. 1, 4.1.3

[24] Ashtekar, A., Engle, J., Pawlowski, T., and van den Broeck, C., "Multipole moments of isolated horizons", Class. Quantum Grav., 21, 2549-2570, (2004). For a related online version see: A. Ashtekar, et al., (January, 2004), [Online Los Alamos Archive Preprint]: cited on 22 November 2004,

http://arXiv.org/abs/gr-qc/0401114. 1, 2.1.3, 4.1.1, 5.3, 7.2, 8

[25] Ashtekar, A., Engle, J., and Van den Broek, C., "Quantum geometry of isolated horizons and black hole entropy: Inclusion of distortion and rotation", (December, 2004), [Online Los Alamos Archive Preprint]: cited on 13 December 2004, http://arXiv.org/abs/gr-qc/0412003. 1,7.3

[26] Ashtekar, A., Fairhurst, S., and Krishnan, B., "Isolated horizons: Hamiltonian evolution and the first law", Phys. Rev. D, 62, 104025-1-29, (2000). For a related online version see: A. Ashtekar, et al., (2000), [Online Los Alamos Archive Preprint]: cited on 22 November 2004 ,

http://arXiv.org/abs/gr-qc/0005083. 1, 2.1, 2.1.1, 2.1.3, 4.1, 4.1.1, 4.1.2, 4.1.3, 4.3, 6, 6.1.1, 6.1.2, 6.2.1, 6.2.1

[27] Ashtekar, A., and Galloway, G., in preparation, (2004). 2.2.3

[28] Ashtekar, A., Hayward, S.A., and Krishnan, B., in preparation. 3.3

[29] Ashtekar, A., and Krasnov, K., "Quantum Geometry and Black Holes", in Iyer, B., and Bhawal, B., eds., Black Holes, Gravitational Radiation and the Universe: Essays in Honor of C.V. Vishveshwara, volume 100 of Fundamental Theories of Physics, 149-170, (Kluwer, Dordrecht, Netherlands; Boston, U.S.A., 1999). For a related online version see: A. Ashtekar, et al., (April, 1998), [Online Los Alamos Archive Preprint]: cited on 22 November 2004, http://arXiv.org/abs/gr-qc/9804039. 7.3, 8

[30] Ashtekar, A., and Krishnan, B., "Dynamical Horizons: Energy, Angular Momentum, Fluxes, and Balance Laws", Phys. Rev. Lett., 89, 261101-1-4, (2002). For a related online version see: A. Ashtekar, et al., (2002), [Online Los Alamos Archive Preprint]: cited on 22 November 2004 ,

http://arXiv.org/abs/gr-qc/0207080. 1, 2.2.1

[31] Ashtekar, A., and Krishnan, B., "Dynamical horizons and their properties", Phys. Rev. D, 68, 104030-1-25, (2003). For a related online version see: A. Ashtekar, et al., (2003), [Online Los Alamos Archive Preprint]: cited on 22 November 2004,

http://arXiv .org/abs/gr-qc/0308033. 1, 2.2.1, 2.2.1, 2.2.1, 2.2.2, 2.2.2, 3.3, 4.2.2, 4.2.2, $4.3,5,8$

[32] Ashtekar, A., and Lewandowski, J., "Background independent quantum gravity: A status report", Class. Quantum Grav., 21, R53-R152, (2004). For a related online version see: A. Ashtekar, et al., (April, 2004), [Online Los Alamos Archive Preprint]: cited on 22 November 2004 ,

http://arXiv.org/abs/gr-qc/0404018. 7.2, 7.2 
[33] Ashtekar, A., and Streubel, M., "Symplective geometry of radiative fields at null infinity", Proc. R. Soc. London, Ser. A, 376, 585-607, (1981). 3.1, 4.1.2, 8

[34] Baiotti, L., Hawke, I., Montero, P.J., Löffler, F., Rezzolla, L., Stergioulas, N., Font, J.A., and Seidel, E., "Three-dimensional relativistic simulations of rotating neutron star collapse to a black hole", (March, 2004), [Online Los Alamos Archive Preprint]: cited on 22 November 2004 , http://arXiv.org/abs/gr-qc/0403029. 1, 2.1, 3.3, 4.2.3, 5

[35] Bardeen, J.M., Carter, B., and Hawking, S.W., "The four laws of black hole mechanics", Commun. Math. Phys., 31, 161-170, (1973). 1, 1

[36] Barreira, M., Carfora, M., and Rovelli, C., "Physics with non-perturbative quantum gravity: Radiation from a quantum black hole", Gen. Relativ. Gravit., 28, 1293-1299, (1996). 7.3

[37] Bartnik, R., and Isenberg, J.A., "Summary of spherically symmetric dynamical horizons", Personal communication to A. Ashtekar. 8

[38] Bartnik, R., and McKinnon, J., "Particlelike solutions of the Einstein-Yang-Mills Equations", Phys. Rev. Lett., 61, 141-144, (1988). 1, 6.1.2

[39] Baumgarte, T.W., "Innermost stable circular orbit of binary black holes", Phys. Rev. D, 62, 024018-1-8, (July, 2000). For a related online version see: T.W. Baumgarte, (April, 2000), [Online Los Alamos Archive Preprint]: cited on 22 November 2004, http://arXiv.org/abs/gr-qc/0004050. 5.2 .2

[40] Beig, R., "The multipole expansion in general relativity", Acta Phys. Austriaca, 53, 249-270, (1981). $\quad 5.3$

[41] Beig, R., and Simon, W., "Proof of a multipole conjecture due to Geroch", Commun. Math. Phys., 78, 1163-1171, (1980). 5.3

[42] Beig, R., and Simon, W., "On the multipole expansion of stationary spacetimes", Proc. R. Soc. London, Ser. A, 376, 333-341, (1981). 5.3

[43] Beig, R., and Simon, W., "The multipole structure of stationary spacetimes", J. Math. Phys., 24, 1163-1171, (1983). 5.3

[44] Bekenstein, J.D., "Black Holes and Entropy", Phys. Rev. D, 7, 2333-2346, (1973). 1, 7.1

[45] Bekenstein, J.D., "Generalized second law of thermodynamics in black-hole physics", Phys. Rev. D, 9, 3292-3300, (1974). 1, 7.1

[46] Bekenstein, J.D., and Meisels, A., "Einstein A and B coefficients for a black hole", Phys. Rev. D, 15, 2775-2781, (1977). 1

[47] Ben-Dov, I., "The Penrose inequality and apparent horizons", (August, 2004), [Online Los Alamos Archive Preprint]: cited on 22 November 2004, http://arXiv.org/abs/gr-qc/0408066. 2.2.2, 8

[48] Beyer, F., Krishnan, B., and Schnetter, E., in preparation. 1, 2.1, 2.2.2, 5, 5.3, 8

[49] Bizoń, P., “Colored Black Holes", Phys. Rev. Lett., 64, 2844-2847, (1990). 1

[50] Bizoń, P., and Chmaj, T., "Gravitating skyrmions", Phys. Lett. B, 297, 55-62, (1992). 6.2.3 
[51] Bizoń, P., and Chmaj, T., "Remark on formation of colored black holes via fine-tuning", Phys. Rev. D, 61, 067501-1-2, (2000). For a related online version see: P. Bizoń, et al., (June, 1999), [Online Los Alamos Archive Preprint]: cited on 22 November 2004, http://arXiv.org/abs/gr-qc/9906070. 6.2 .2

[52] Bizoń, P., and Wald, R.M., "The $\mathrm{n}=1$ colored black hole is unstable", Phys. Lett. B, 267, 173-174, (1991). $\quad 6.2 .2$

[53] Blackburn, J.K., and Detweiler, S., "Close black-hole binary systems", Phys. Rev. D, 46, 2318-2333, (1992). $\quad 5.2 .1$

[54] Bondi, H., van der Burg, M.G.J., and Metzner, A.W.K., "Gravitational waves in general relativity VII: Waves from axi-symmetric isolated systems", Proc. R. Soc. London, Ser. A, 269, 21, (1962). $3.1,5.4,8$

[55] Booth, I., "Metric-based Hamiltonians, null boundaries, and isolated horizons", Class. Quantum Grav., 18, 4239-4264, (2001). For a related online version see: I. Booth, (May, 2001), [Online Los Alamos Archive Preprint]: cited on 22 November 2004,

http://arXiv.org/abs/gr-qc/0105009. 4.1.2, 4.1.2

[56] Booth, I., and Fairhurst, S., "The First Law for Slowly Evolving Horizons", Phys. Rev. Lett., 92, 011102-1-4, (2004). For a related online version see: I. Booth, et al., (2003), [Online Los Alamos Archive Preprint]: cited on 22 November 2004,

http://arXiv.org/abs/gr-qc/0307087. 1, 4.2.2, 4.3

[57] Bowen, J.M., and York Jr, J.W., "Time-asymmetric initial data for black holes and black-hole collisions", Phys. Rev. D, 21, 2047-2056, (1980). 5.2.2

[58] Brandt, S.R., Correll, R.R., Gómez, R., Huq, M.F., Laguna, P., Lehner, L., Marronetti, P., Matzner, R., Neilsen, D., Pullin, J., Schnetter, E., Shoemaker, D.M., and Winicour, J., "Grazing collision of black holes via the excision of singularities", Phys. Rev. Lett., 85, 5496-5499, (2000). For a related online version see: S.R. Brandt, et al., (September, 2000), [Online Los Alamos Archive Preprint]: cited on 22 November 2004, http://arXiv.org/abs/gr-qc/0009047. 1

[59] Bray, H., "Proof of the Riemannian Penrose inequality using the positive mass theorem", $J$. Differ. Geom., 59, 177, (2001). 8

[60] Breitenlohner, P., Forgács, P., and Maison, D., "On static spherically symmetric solutions of the Einstein-Yang-Mills equations", Commun. Math. Phys., 163, 141-172, (1994). 6.1 .2

[61] Breitenlohner, P., Forgács, P., and Maison, D., "Gravitating monopole solutions II", Nucl. Phys. B, 442, 126-156, (1995). 6.2 .2

[62] Bretón, N., "Born-Infeld black hole in the isolated horizon framework", Phys. Rev. D, 67, 124004-1-4, (2003). For a related online version see: N. Bretón, (January, 2003), [Online Los Alamos Archive Preprint]: cited on 22 November 2004, http://arXiv.org/abs/gr-qc/hep-th/0301254. 6.2.1

[63] Brill, D.R., and Lindquist, R.W., "Interaction Energy in Geometrostatics", Phys. Rev., 131, 471-476, (1963). $\quad 5.2 .2$

[64] Brügmann, B., Tichy, W., and Jansen, N., "Numerical Simulation of Orbiting Black Holes", Phys. Rev. Lett., 92, 211101, (2004). For a related online version see: B. Brügmann, et al., (December, 2003), [Online Los Alamos Archive Preprint]: cited on 22 November 2004, http://arXiv.org/abs/gr-qc/0312112. 5 
[65] Carter, B., "Black hole equilibrium states", in DeWitt, C., and DeWitt, B.S., eds., Black Holes: Based on lectures given at the 23rd session of the Summer School of Les Houches, 1972, 57-214, (Gordon and Breach, New York, U.S.A., 1973). 1

[66] Chandrasekhar, S., The Mathematical Theory of Black Holes, volume 69 of The International Series of Monographs on Physics, (Clarendon Press, Oxford, U.K., 1983). 1, 3.3

[67] Choptuik, M.W., "Universality and scaling in gravitational collapse of a massless scalar field", Phys. Rev. Lett., 70, 9-12, (1993). 1

[68] Chruściel, P.T., "On the global structure of Robinson-Trautman space-time", Proc. R. Soc. London, Ser. A, 436, 299-316, (1992). 1, 2.1.1, 2.1.2

[69] Chruściel, P.T., "No Hair Theorems Folklore, Conjectures, Results", in Beem, J.K., and Duggal, K.L., eds., Differential Geometry and Mathematical Physics: AMS-CMS Special Session on Geometric Methods in Mathematical Physics, August 15-19, 1993, Vancouver, British Columbia, Canada, volume 170 of Contemporary Mathematics, 23-49, (American Mathematical Society, Providence, U.S.A., 1994). 1

[70] Cook, G.B., "Initial Data for Numerical Relativity", Living Rev. Relativity, 2, (2000), [Online Journal Article]: cited on 22 November 2004, http://www. livingreviews.org/lrr-2000-5. 5.2, 5.2.1

[71] Cook, G.B., "Three-dimensional initial data for the collision of two black holes II: Quasicircular orbits for equal mass black holes", Phys. Rev. D, 50, 5025-5032, (October, 1994). For a related online version see: G.B. Cook, (April, 1994), [Online Los Alamos Archive Preprint]: cited on 22 November 2004,

http://arXiv.org/abs/gr-qc/9404043. 5.2 .2

[72] Cook, G.B., "Corotating and irrotational binary black holes in quasicircular orbits", Phys. Rev. D, 65, 084003-1-13, (2002). For a related online version see: G.B. Cook, (August, 2001), [Online Los Alamos Archive Preprint]: cited on 22 November 2004, http://arXiv.org/abs/gr-qc/0108076. 1, 5.2, 5.2.1, 5.2.1, 6

[73] Cook, G.B., Huq, M.F., Klasky, S.A., Scheel, M.A., Abrahams, A.M., Anderson, A., Anninos, P., Baumgarte, T.W., Bishop, N.T., Brandt, S.R., Browne, J.C., Camarda, K., Choptuik, M.W., Evans, C.R., Finn, L.F., Fox, G.C., Gómez, R., Haupt, T., Kidder, L.E., Laguna, P., Landry, W., Lehner, L., Lenaghan, J., Marsa, R.L., Massó, J., Matzner, R.A., Mitra, S., Papadopoulos, P., Parashar, M., Rezzolla, L., Rupright, M.E., Saied, F., Saylor, P.E., Seidel, E., Shapiro, S.L., Shoemaker, D.M., Smarr, L.L., Suen, W.-M., Szilágyi, B., Teukolsky, S.A., van Putten, M.H.P.M., Walker, P., Winicour, J., and York Jr, J.W. (Binary Black Hole Grand Challenge Alliance), "Boosted Three-Dimensional Black-Hole Evolutions with Singularity Excision", Phys. Rev. Lett., 80, 2512-2516, (1998). For a related online version see: G.B. Cook, et al., (November, 1997), [Online Los Alamos Archive Preprint]: cited on 22 November 2004, http://arXiv.org/abs/gr-qc/9711078. Binary Black Hole Grand Challenge Alliance. 5, 5.2 .1

[74] Cook, G.B., and Pfeiffer, H.P., "Excision boundary conditions for black-hole initial data", Phys. Rev. D, 70, 104016-1-24, (2004). 5.2.1

[75] Corichi, A., Nucamendi, U., and Sudarsky, D., "Einstein-Yang-Mills isolated horizons: Phase space, mechanics, hair, and conjectures", Phys. Rev. D, 62, 044046-1-19, (2000). For a related online version see: A. Corichi, et al., (February, 2000), [Online Los Alamos

Living Reviews in Relativity

http://www. livingreviews . org//rr-2004-10 
Archive Preprint]: cited on 22 November 2004,

http://arXiv.org/abs/gr-qc/0002078. 1, 4.1, 6, 6.2.1, 8

[76] Corichi, A., Nucamendi, U., and Sudarsky, D., "Mass formula for Einstein-Yang-Mills solitons", Phys. Rev. D, 64, 107501-1-4, (2001). For a related online version see: A. Corichi, et al., (June, 2001), [Online Los Alamos Archive Preprint]: cited on 22 November 2004, http://arXiv .org/abs/gr-qc/0106084. 4.1, 6, 6.2.3, 6.2.3

[77] Corichi, A., and Sudarsky, D., "Mass of colored black holes", Phys. Rev. D, 61, 101501-1-4, (2000). For a related online version see: A. Corichi, et al., (December, 1999), [Online Los Alamos Archive Preprint]: cited on 22 November 2004,

http://arXiv .org/abs/gr-qc/9912032. 4.1, 6, 6.2.1, 6.2.1, 6.2.1

[78] Cutler, C., and Thorne, K.S., "An Overview of Gravitational-Wave Sources", in Bishop, N.T., and Maharaj, S.D., eds., General Relativity and Gravitation: Proceedings of the 16th International Conference on General Relativity and Gravitation, Durban, South Africa, 1521 July 2001, 72-111, (World Scientific, Singapore; River Edge, U.S.A., 2002). For a related online version see: C. Cutler, et al., (April, 2002), [Online Los Alamos Archive Preprint]: cited on 22 November 2004,

http://arXiv.org/abs/gr-qc/0204090. 1,5.4

[79] Dain, S., "Black hole interaction energy", Phys. Rev. D, 66, 084019-1-8, (2002). For a related online version see: S. Dain, (July, 2002), [Online Los Alamos Archive Preprint]: cited on 22 November 2004,

http://arXiv.org/abs/gr-qc/0207090. 5.2 .2

[80] Dain, S., "Trapped surfaces as boundaries for the constraint equations", Class. Quantum Grav., 21, 555-574, (2004). For a related online version see: S. Dain, (August, 2003), [Online Los Alamos Archive Preprint]: cited on 22 November 2004, http://arXiv.org/abs/gr-qc/0308009. 6

[81] Dain, S., Jaramillo, J.L., and Krishnan, B. 1, 6

[82] Diener, P., personal communication to B. Krishnan. 5.4

[83] Diener, P., "A new general purpose event horizon finder for 3D numerical spacetimes", Class. Quantum Grav., 20, 4901-4918, (2003). For a related online version see: P. Diener, (2003), [Online Los Alamos Archive Preprint]: cited on 22 November 2004, http://arXiv.org/abs/gr-qc/0305039. 5.4

[84] Domagala, M., and Lewandowski, J., "Black-hole entropy from quantum geometry", Class. Quantum Grav., 21, 5233-5243, (2004). For a related online version see: M. Domagala, et al., (2004), [Online Los Alamos Archive Preprint]: cited on 22 November 2004, http://arXiv.org/abs/gr-qc/0407051. 1,7.3

[85] Dreyer, O., Krishnan, B., Schnetter, E., and Shoemaker, D.M., "Introduction to isolated horizons in numerical relativity", Phys. Rev. D, 67, 024018-1-14, (2003). For a related online version see: O. Dreyer, et al., (June, 2002), [Online Los Alamos Archive Preprint]: cited on 22 November 2004, http://arXiv .org/abs/gr-qc/0206008. 1, 5.1, 5.1, 5, 5

[86] Eardley, D.M., "Black Hole Boundary Conditions and Coordinate Conditions", Phys. Rev. $D, \mathbf{5 7}, 2299-2304$, (1998). For a related online version see: D.M. Eardley, (1997), [Online Los Alamos Archive Preprint]: cited on 22 November 2004, http://arXiv.org/abs/gr-qc/9703027. 8 
[87] Ernst, F.J., "Black holes in a magnetic universe", J. Math. Phys., 17, 54-56, (1976). 1

[88] Fairhurst, S., and Krishnan, B., "Distorted black holes with charge", Int. J. Mod. Phys. D, 10, 691-710, (2001). For a related online version see: S. Fairhurst, et al., (October, 2000), [Online Los Alamos Archive Preprint]: cited on 22 November 2004, http://arXiv.org/abs/gr-qc/0010088. 1, 5.3

[89] Finn, L.S., Personal communication to A. Ashtekar. 1, 8

[90] Friedman, J.L., Schleich, K., and Witt, D.M., "Topological censorship", Phys. Rev. Lett., 71, 1486-1489, (1993). For a related online version see: J.L. Friedman, et al., (May, 1993), [Online Los Alamos Archive Preprint]: cited on 22 November 2004, http://arXiv.org/abs/gr-qc/9305017. Erratum: Phys. Rev. Lett. 75 (1995) 1872.1

[91] Friedman, J.L., Uryū, K., and Shibata, M., "Thermodynamics of binary black holes and neutron stars", Phys. Rev. D, 65, 064035-1-20, (2002). For a related online version see: J.L. Friedman, et al., (June, 2001), [Online Los Alamos Archive Preprint]: cited on 22 November 2004 , http://arXiv.org/abs/gr-qc/0108070. 5.2 .1

[92] Friedrich, H., "On the regular and asymptotic characteristic initial value problem for Einstein's field equations", Proc. R. Soc. London, Ser. A, 375, 169-184, (1981). 2.1 .2

[93] Galloway, G.J., personal communication to A. Ashtekar, (2004). 2.2 .3

[94] Gambini, R., Obregón, O., and Pullin, J., "Yang-Mills analogs of the Immirzi ambiguity", Phys. Rev. D, 59, 047505-1-4, (1999). For a related online version see: R. Gambini, et al., (2001), [Online Los Alamos Archive Preprint]: cited on 22 November 2004, http://arXiv.org/abs/gr-qc/9801055. 7.2

[95] Garfinkle, D., Horowitz, G.T., and Strominger, A., "Charged black holes in string theory", Phys. Rev. D, 43, 3140-3143, (1991). 6.1.1

[96] Garfinkle, D., Horowitz, G.T., and Strominger, A., "Erratum: Charged black holes in string theory", Phys. Rev. D, 45, 3888, (1992). 6.1.1

[97] Geroch, R., "Multipole moments II. Curved space", J. Math. Phys., 11, 2580-2588, (1970). 5.3

[98] Geroch, R., and Hartle, J.B., "Distorted Black Holes", J. Math. Phys., 23, 680, (1982). 1, 5.3

[99] Gibbons, G.W., and Hawking, S.W., "Cosmological event horizons, thermodynamics, and particle creation", Phys. Rev. D, 15, 2738-2751, (1977). 1, 7.3

[100] Gibbons, G.W., Kallosh, R.E., and Kol, B., "Moduli, Scalar Charges, and the First Law of Black Hole Thermodynamics", Phys. Rev. Lett., 77, 4992-4995, (1996). 6.1.1

[101] Gibbons, G.W., and Maeda, K., "Black holes and membranes in higher-dimensional theories with dilaton fields", Nucl. Phys. B, 298, 741-775, (1998). 6.1.1

[102] Gonzalez, J., and van den Broeck, C., in preparation. 2.2.2, 8

Living Reviews in Relativity

http: //www . livingreviews . org/Irr-2004-10 
[103] Gourgoulhon, E., Grandclément, P., and Bonazzola, S., "Binary black holes in circular orbits. I. A global spacetime approach", Phys. Rev. D, 65, 044020-1-19, (2002). For a related online version see: E. Gourgoulhon, et al., (June, 2001), [Online Los Alamos Archive Preprint]: cited on 22 November 2004,

http://arXiv.org/abs/gr-qc/0106015. 5.2 .1

[104] Grandclément, P., Gourgoulhon, E., and Bonazzola, S., "Binary black holes in circular orbits. II. Numerical methods and first results", Phys. Rev. D, 65, 044021-1-18, (2002). For a related online version see: P. Grandclément, et al., (June, 2001), [Online Los Alamos Archive Preprint]: cited on 22 November 2004, http://arXiv.org/abs/gr-qc/0106016. 5.2 .1

[105] Gundlach, C., "Critical phenomena in gravitational collapse", Adv. Theor. Math. Phys., 2, 1-49, (1998). For a related online version see: C. Gundlach, (December, 1997), [Online Los Alamos Archive Preprint]: cited on 22 November 2004,

http://arXiv.org/abs/gr-qc/9712084. 1

[106] Hájíček, P., "Stationary electrovacuum spacetimes with bifurcate horizons", J. Math. Phys., 16, 518-522, (1975). 2.1

[107] Hansen, R., "Multipole moments in stationary space-times", J. Math. Phys., 15, 46-52, (1974). $\quad 1,5.3$

[108] Hartle, J.B., and Hawking, S.W., "Energy and Angular Momentum Flow in to a Black Hole", Commun. Math. Phys., 27, 283-290, (1972). 3.3

[109] Hartmann, B., Kleihaus, B., and Kunz, J., "Axially symmetric monopoles and black holes in Einstein-Yang-Mills-Higgs theory", Phys. Rev. D, 65, 024027-1-22, (2002). For a related online version see: B. Hartmann, et al., (2001), [Online Los Alamos Archive Preprint]: cited on 22 November 2004,

http://arXiv.org/abs/hep-th/0108129. 6.2.1, 6.2.2, 8

[110] Hawking, S.W., "Black Holes in General Relativity", Commun. Math. Phys., 25, 152, (1972). 1

[111] Hawking, S.W., "The event horizon", in DeWitt, C., and DeWitt, B.S., eds., Black Holes: Based on lectures given at the 23rd session of the Summer School of Les Houches, 1972, 1-56, (Gordon and Breach, New York, U.S.A., 1973). 1, 2.2.1

[112] Hawking, S.W., "Particle Creation by Black Holes", Commun. Math. Phys., 43, 199, (1975). 7.1

[113] Hawking, S.W., and Ellis, G.F.R., The Large Scale Structure of Space-Time, Cambridge Monographs on Mathematical Physics, (Cambridge University Press, Cambridge, U.K., 1973). $\quad 1,2.2 .1$

[114] Hawking, S.W., and Hunter, C.J., "Gravitational entropy and global structure", Phys. Rev. $D$, 59, 044025-1-10, (1999). For a related online version see: S.W. Hawking, et al., (1998), [Online Los Alamos Archive Preprint]: cited on 22 November 2004,

http://arXiv.org/abs/hep-th/9808085. 8

[115] Hayward, S., "Energy and entropy conservation for dynamical black holes", Phys. Rev. D, 70, 104027-1-13, (2004). For a related online version see: S. Hayward, (August, 2004), [Online Los Alamos Archive Preprint]: cited on 22 November 2004, http://arXiv.org/abs/gr-qc/0408008. 1 
[116] Hayward, S.A., "Energy conservation for dynamical black holes", (April, 2004), [Online Los Alamos Archive Preprint]: cited on 22 November 2004,

http://arXiv.org/abs/gr-qc/0404077. 1

[117] Hayward, S.A., "General laws of black-hole dynamics", Phys. Rev. D, 49, 6467-6474, (1994). For a related online version see: S.A. Hayward, (1993), [Online Los Alamos Archive Preprint]: cited on 22 November 2004,

http://arXiv.org/abs/gr-qc/9303006. 1, 2.2.1, 2.2.1, 3.3, 8

[118] Hayward, S.A., "Spin-Coefficient Form of the New Laws of Black-Hole Dynamics", Class. Quantum Grav., 11, 3025-3036, (1994). For a related online version see: S.A. Hayward, (1994), [Online Los Alamos Archive Preprint]: cited on 22 November 2004, http://arXiv.org/abs/gr-qc/9406033.

[119] Heusler, M., Black Hole Uniqueness Theorems, (Cambridge University Press, Cambridge, U.K.; New York, U.S.A., 1996). 1

[120] Horowitz, G.T., "Quantum States of Black Holes", in Wald, R.M., ed., Black Holes and Relativistic Stars, 241-266, (University of Chicago Press, Chicago, U.S.A., 1998). 1

[121] Hughes, S.A., Keeton II, C.R., Walker, P., Walsh, K.T., Shapiro, S.L., and Teukolsky, S.A., "Finding black holes in numerical spacetimes", Phys. Rev. D, 49, 4004-4015, (1994). 3.2

[122] Huisken, G., and Ilmanen, T., "The inverse mean curvature flow and the Riemannian Penrose inequality", J. Differ. Geom., 59, 353, (2001). 8

[123] Jacobson, T., Kang, G., and Myers, R.C., "On black hole entropy", Phys. Rev. D, 49, $6587-6598,(1994) . \quad 6,7.3$

[124] Jaramillo, J.L., Gourgoulhon, E., and Mena Marugán, G.A., "Inner boundary conditions for black hole Initial Data derived from Isolated Horizons", (2004), [Online Los Alamos Archive Preprint]: cited on 22 November 2004, http://arXiv .org/abs/gr-qc/0407063. 1, 2, 5.2, 5.2.1, 5.2.1, 6

[125] Kastor, D., and Traschen, J., "Cosmological multi-black-hole solutions", Phys. Rev. D, 47, 5370-5375, (1993). $\quad 2.1 .2$

[126] Khanna, G., Baker, J., Gleiser, R.J., Laguna, P., Nicasio, C.O., Nollert, H.-P., Price, R.H., and Pullin, J., "Inspiraling Black Holes: The Close Limit", Phys. Rev. Lett., 83, 3581-3584, (1999). For a related online version see: G. Khanna, et al., (May, 1999), [Online Los Alamos Archive Preprint]: cited on 22 November 2004, http://arXiv.org/abs/gr-qc/9905081. 1

[127] Kleihaus, B., and Kunz, J., "Static Black-Hole Solutions with Axial Symmetry", Phys. Rev. Lett., 79, 1595-1598, (1997). 6.1.2, 6.2.1

[128] Kleihaus, B., and Kunz, J., "Static axially symmetric Einstein-Yang-Mills-Dilaton solutions: 1. Regular solutions", Phys. Rev. D, 57, 843-856, (1998). 6.1 .2

[129] Kleihaus, B., and Kunz, J., "Static axially symmetric Einstein-Yang-Mills-Dilaton solutions: 2. Black hole solutions", Phys. Rev. D, 57, 6138-6157, (1998). 6.1 .2

[130] Kleihaus, B., and Kunz, J., "Non-Abelian black holes with magnetic dipole hair", Phys. Lett. $B, 494,130-134,(2000)$. For a related online version see: B. Kleihaus, et al., (2000), [Online Los Alamos Archive Preprint]: cited on 22 November 2004, http://arXiv.org/abs/hep-th/0008034. 6.2.1 
[131] Kleihaus, B., Kunz, J., and Navarro-Lérida, F., "Rotating dilaton black holes with hair", Phys. Rev. D, 69, 064028-1-30, (2004). For a related online version see: B. Kleihaus, et al., (June, 2003), [Online Los Alamos Archive Preprint]: cited on 22 November 2004, http://arXiv.org/abs/gr-qc/0306058. 8

[132] Kleihaus, B., Kunz, J., Sood, A., and Wirschins, M., "Horizon properties of Einstein-YangMills black hole", Phys. Rev. D, 65, 061502-1-4, (2002). 6.2.1, 6.2.2, 8

[133] Korzynski, N., Lewandowski, J., and Pawlowski, T., "Mechanics of isolated horizons in higher dimensions", in preparation. 4.1 .3

[134] Krasnov, K., "Geometrical entropy from loop quantum gravity", Phys. Rev. D, 55, 35053513, (1997). 7.3

[135] Krasnov, K., "On statistical mechanics of Schwarzschild black holes", Gen. Relativ. Gravit., 30, 53-68, (1998). 7.3

[136] Krishnan, B., Isolated Horizons in Numerical Relativity, PhD Thesis, (The Pennsylvania State University, University Park, U.S.A., 2002). For a related online version see: B. Krishnan, (2002), [Online Thesis]: cited on 22 November 2004,

http://etda.libraries.psu.edu/theses/approved/WorldWideIndex/ETD-177/. 1, 2.1.1, 5.2.2, 5.2.2

[137] Kuroda, Y., "Naked Singularities in the Vaidya Spacetimee", Prog. Theor. Phys., 72, 63-72, (1984). $\quad 2.2 .2,4$

[138] Lehner, L., "Numerical Relativity: A review", Class. Quantum Grav., 18, R25-R86, (2001). For a related online version see: L. Lehner, (June, 2001), [Online Los Alamos Archive Preprint]: cited on 22 November 2004, http://arXiv.org/abs/gr-qc/0106072. 1, 5.4

[139] Lehner, L., Bishop, N.T., Gómez, R., Szilágyi, B., and Winicour, J., "Exact solutions for the intrinsic geometry of black hole coalescence", Phys. Rev. D, 60, 044005-1-10, (1999). For a related online version see: L. Lehner, et al., (September, 1998), [Online Los Alamos Archive Preprint]: cited on 22 November 2004, http://arXiv.org/abs/gr-qc/9809034. 3.2

[140] Lewandowski, J., "Spacetimes admitting isolated horizons", Class. Quantum Grav., 17, L53L59, (2000). For a related online version see: J. Lewandowski, (July, 1999), [Online Los Alamos Archive Preprint]: cited on 22 November 2004,

http://arXiv.org/abs/gr-qc/9907058. 2.1.2

[141] Lewandowski, J., and Pawlowski, T., "Quasi-local rotating black holes in higher dimension: geometry", (October, 2004), [Online Los Alamos Archive Preprint]: cited on 22 November 2004,

http://arXiv.org/abs/gr-qc/0410146. 1

[142] Lewandowski, J., and Pawlowski, T., "Geometric characterizations of the Kerr isolated horizon", Int. J. Mod. Phys. D, 11, 739-746, (2001). For a related online version see: J. Lewandowski, et al., (December, 2001), [Online Los Alamos Archive Preprint]: cited on 22 November 2004,

http://arXiv.org/abs/gr-qc/0101008. 1 
[143] Lewandowski, J., and Pawlowski, T., "Extremal isolated horizons: a local uniqueness theorem", Class. Quantum Grav., 20, 587-606, (2003). For a related online version see: J. Lewandowski, et al., (August, 2002), [Online Los Alamos Archive Preprint]: cited on 22 November 2004, http://arXiv.org/abs/gr-qc/0208032. 2.1.3, 5.3

[144] Lichnerowicz, A., "L'integration des équations de la gravitation relativiste et le probléme des n corps", J. Math. Pures Appl., 23, 37-63, (1944). 5.2.1

[145] Maldacena, J., and Strominger, A., "Statistical entropy of four-dimensional extremal black holes", Phys. Rev. Lett., 77, 428-429, (1996). For a related online version see: J. Maldacena, et al., (March, 1996), [Online Los Alamos Archive Preprint]: cited on 22 November 2004, http://arXiv.org/abs/hep-th/9603060. 1

[146] Mann, R.B., "Misner string entropy", Phys. Rev. D, 60, 104047-1-5, (1999). For a related online version see: R.B. Mann, (March, 1999), [Online Los Alamos Archive Preprint]: cited on 22 November 2004, http://arXiv.org/abs/hep-th/9903229. 8

[147] Mann, R.B., and Garfinkle, D., "Generalized entropy and Noether charge", Class. Quantum Grav., 17, 3317-3324, (2000). For a related online version see: R.B. Mann, et al., (2000), [Online Los Alamos Archive Preprint]: cited on 22 November 2004, http://arXiv.org/abs/gr-qc/0004056. 8

[148] Masood-ul Alam, A.K.M., "Uniqueness of a static charged dilaton black hole", Class. Quantum Grav., 10, 2649-2656, (1993). 6.1.1

[149] Meissner, K.A., "Black-hole entropy in loop quantum gravity", Class. Quantum Grav., 21, 5245-5252, (2004). For a related online version see: K.A. Meissner, (July, 2004), [Online Los Alamos Archive Preprint]: cited on 22 November 2004, http: //arXiv.org/abs/gr-qc/0407052. 1, 7.3

[150] Misner, C.W., "Wormhole Initial Conditions", Phys. Rev., 118, 1110-1111, (1959). 5.2 .2

[151] Misner, C.W., "The method of images in geometrostatics", Ann. Phys. (N.Y.), 24, 102-117, (1963). $\quad 5.2 .2$

[152] Nakao, K., Shiromizu, T., and Hayward, S.A., "Horizons of the Kastor-Traschen multi-blackhole cosmos", Phys. Rev. D, 52, 796-808, (1995). 2.1 .2

[153] New, K.C.B., "Gravitational Waves from Gravitational Collapse", Living Rev. Relativity, 6, (2003), [Online Journal Article]: cited on 22 November 2004, http://www. livingreviews.org/lrr-2003-2. 1, 5.4

[154] Núñez, D., Quevedo, H., and Sudarsky, D., "Black Holes have no Short Hair", Phys. Rev. Lett., 76, 571-574, (1996). For a related online version see: D. Núñez, et al., (January, 1996), [Online Los Alamos Archive Preprint]: cited on 22 November 2004, http://arXiv.org/abs/gr-qc/9601020. 6.2 .3

[155] Pawlowski, T., Lewandowski, J., and Jezierski, J., "Spacetimes foliated by Killing horizons", Class. Quantum Grav., 21, 1237-1252, (2004). For a related online version see: T. Pawlowski, et al., (June, 2003), [Online Los Alamos Archive Preprint]: cited on 22 November 2004, http://arXiv.org/abs/gr-qc/0306107. 2.1.2 
[156] Pejerski, D.W., and Newman, E.T., "Trapped surface and the development of singularities", J. Math. Phys., 9, 1929-1937, (1971). 2.1

[157] Penrose, R., "Naked singularities", Ann. N.Y. Acad. Sci., 224, 125-134, (1973). 1, 3.1

[158] Pfeiffer, H.P., Cook, G.B., and Teukolsky, S.A., "Comparing initial-data sets for binary black holes", Phys. Rev. D, 66, 024047-1-17, (2002). For a related online version see: H.P. Pfeiffer, et al., (June, 2002), [Online Los Alamos Archive Preprint]: cited on 22 November 2004, http://arXiv.org/abs/gr-qc/0203085. 5.2.2

[159] Pfeiffer, H.P., Teukolsky, S.A., and Cook, G.B., "Quasicircular orbits for spinning binary black holes", Phys. Rev. D, 62, 104018-1-11, (2000). For a related online version see: H.P. Pfeiffer, et al., (June, 2000), [Online Los Alamos Archive Preprint]: cited on 22 November 2004 ,

http://arXiv.org/abs/gr-qc/0006084. 5.2 .2

[160] Pullin, J., "The close limit of colliding black holes: An update", Prog. Theor. Phys. Suppl., 136, 107-120, (1999). For a related online version see: J. Pullin, (September, 1999), [Online Los Alamos Archive Preprint]: cited on 22 November 2004,

http://arXiv.org/abs/gr-qc/9909021. 1

[161] Rendall, A.D., "Reduction of the characteristic initial value problem to the Cauchy problem and its applications to the Einstein equations", Proc. R. Soc. London, Ser. A, 427, 221-239, (1990). $\quad 2.1 .2$

[162] Rovelli, C., "Loop Quantum Gravity", Living Rev. Relativity, 1, (1998), [Online Journal Article]: cited on 22 November 2004,

http://www.livingreviews.org/lrr-1998-1. 7.2

[163] Rovelli, C., "Black hole entropy from loop quantum gravity", Phys. Rev. Lett., 14, 32883291, (1996). 7.3

[164] Rovelli, C., "Loop quantum gravity and black hole physics", Helv. Phys. Acta, 69, 582-611, (1996). $\quad 7.3$

[165] Sachs, R.K., and Bergmann, P.G., "Structure of particles in linearized gravitational theory", Phys. Rev., 112, 674-680, (1958). 5.3

[166] Senovilla, J.M.M., "On the existence of horizons in spacetimes with vanishing curvature invariants", J. High Energy Phys., 11, 046, (2003). For a related online version see: J.M.M. Senovilla, (November, 2003), [Online Los Alamos Archive Preprint]: cited on 22 November 2004,

http://arXiv.org/abs/hep-th/0311172. 2.2.1

[167] Shapiro, S.L., and Teukolsky, S.A., "Collisions of relativistic clusters and the formation of black holes", Phys. Rev. D, 45, 2739-2750, (1992). 3.2

[168] Shoemaker, D.M., Huq, M.F., and Matzner, R.A., "Generic tracking of multiple apparent horizons with level flow", Phys. Rev. D, 62, 124005-1-12, (2000). For a related online version see: D.M. Shoemaker, et al., (April, 2000), [Online Los Alamos Archive Preprint]: cited on 22 November 2004, http://arXiv.org/abs/gr-qc/0004062. 5

[169] Smarr, L.L., "Surface Geometry of Charged Rotating Black Holes", Phys. Rev. D, 7, 289-295, (1973). 5 
[170] Smolin, L., "Linking topological quantum field theory and nonperturbative quantum gravity", J. Math. Phys., 36, 6417-6455, (1995). 7.3

[171] Smoller, J.A., Wasserman, A.G., and Yau, S.T., "Existence of black hole solutions for the Einstein / Yang-Mills equations", Commun. Math. Phys., 154, 377, (1993). 6.1 .2

[172] Straumann, N., and Zhou, Z.H., "Instability of a colored black hole solution", Phys. Lett. B, 243, 33, (1990). 6.2 .2

[173] Straumann, N., and Zhou, Z.H., "Instability of the Bartnik-McKinnon solution to the Einstein-Yang-Mills equations", Phys. Lett. B, 237, 353, (1990). 6.2 .2

[174] Strominger, A., and Vafa, C., "Microscopic origin of the Bekenstein-Hawking entropy", Phys. Lett. B, 379, 99-104, (1996). For a related online version see: A. Strominger, et al., (January, 1996), [Online Los Alamos Archive Preprint]: cited on 22 November 2004, http://arXiv.org/abs/hep-th/9601029. 1

[175] Sudarsky, D., and Wald, R.M., "Extrema of mass, stationarity and staticity, and solutions to the Einstein-Yang-Mills equations", Phys. Rev. D, 46, 1453-1474, (1992). 1

[176] Thiemann, T., "Introduction to modern canonical quantum general relativity", (October, 2001), [Online Los Alamos Archive Preprint]: cited on 22 November 2004, http://arXiv.org/abs/gr-qc/0110034. 7.2

[177] Thornburg, J., "A fast apparent horizon finder for 3-dimensional Cartesian grids in numerical relativity", Class. Quantum Grav., 21, 743-766, (2004). For a related online version see: J. Thornburg, (June, 2003), [Online Los Alamos Archive Preprint]: cited on 22 November 2004 , http://arXiv.org/abs/gr-qc/0306056. 5

[178] Torii, T., and Maeda, K., "Black holes with non-Abelian hair and their thermodynamical properties", Phys. Rev. D, 48, 1643-1651, (1993). 6.2.3

[179] Vaidya, P.C., "The gravitational field of a radiating star", Proc. Indian Acad. Sci., Sect. A, 33, 264, (1951). 2.2 .2

[180] van den Broeck, C., personal communication to A. Ashtekar. 2.2.2

[181] Volkov, M.S., and Gal'tsov, D.V., "Gravitating non-Abelian solitons and black holes with Yang-Mills fields", Phys. Rep., 319, 1, (1999). 1, 6.2.1, 6.2.2, 6.2.3

[182] Wald, R.M., "The Thermodynamics of Black Holes", Living Rev. Relativity, 4, (July, 2001), [Online Journal Article]: cited on 22 November 2004, http://www.livingreviews .org/lrr-2001-6. 1, 2.1.1

[183] Wald, R.M., "Black hole entropy is the Noether charge", Phys. Rev. D, 48, R3427-R3431, (1993). $\quad 6,7.3$

[184] Wald, R.M., and Iyer, V., "Some properties of the Noether charge and a proposal for dynamical black hole entropy", Phys. Rev. D, 50, 846-864, (1994). 6, 7.3

[185] Wald, R.M., and Zoupas, A., "General definition of "conserved quantities" in general relativity and other theories of gravity", Phys. Rev. D, 61, 084027-1-16, (2000). For a related online version see: R.M. Wald, et al., (November, 1999), [Online Los Alamos Archive Preprint]: cited on 22 November 2004,

http://arXiv.org/abs/gr-qc/9911095. 3.1, 4.1.2, 5, 8

Living Reviews in Relativity

http: //www. livingreviews . org/Irr-2004-10 
[186] Waugh, B., and Lake, K., "Double-null coordibates for the Vaidya spacetime", Phys. Rev. D, 34, 2978-2984, (1986). 2.2 .2

[187] Wheeler, J.A., "It from Bit", in Keldysh, L.V., and Feinberg, V.Y., eds., Sakharov Memorial Lectures on Physics: Proceedings of the First International Sakharov Conferenference on Physics, Volume 2, (Nova Science, New York, U.S.A., 1992). 7.3

[188] Witten, E., "A new proof of the positive energy theorem", Commun. Math. Phys., 80, 381402, (1981). 3.3

[189] Wolfram Research, Inc., "Mathematica: The Way the World Calculates", (2004), [Online HTML document]: cited on 22 November 2004,

http://www.wolfram.com/products/mathematica/index.html. $\quad 2.2 .2$

[190] Yo, H.-J., Cook, J.N., Shapiro, S.L., and Baumgarte, T.W., "Quasi-equilibrium binary black hole initial data for dynamical evolutions", Phys. Rev. D, 70, 084033-1-14, (2004). 5.2.1, 6

[191] York Jr, J.W., "Kinematics and Dynamics of General Relativity", in Small, L.L., ed., Sources of Gravitational Radiation: Proceedings of the Battelle Seattle Workshop, July 24 - August 4, 1978, 83-126, (Cambridge University Press, Cambridge, U.K.; New York, U.S.A., 1979). 5.2 .1

[192] York Jr, J.W., "Conformal 'thin-sandwich' data for the initial-value problem of general relativity", Phys. Rev. Lett., 82, 1350-1353, (1999). For a related online version see: J.W. York Jr, (October, 1998), [Online Los Alamos Archive Preprint]: cited on 22 November 2004 ,

http://arXiv.org/abs/gr-qc/9810051. 5.2.1, 5.2.1 